\title{
Design of UV-Absorbing Donor Molecules for Nearly Imperceptible Organic Solar Cells
}

Melissa L. Ball, ${ }^{1, *}$ Quinn Burlingame, ${ }^{1, *}$ Hannah L. Smith, ${ }^{2}$ Tianran Liu, ${ }^{2}$ Sean R. Parkin, ${ }^{3}$ Antoine Kahn, ${ }^{2}$ and Yueh-Lin Loo ${ }^{1,4, \dagger}$

${ }^{1}$ Andlinger Center for Energy and the Environment, Princeton University, Princeton, NJ 08544, USA

${ }^{2}$ Department of Electrical and Computer Engineering, Princeton University, Princeton, NJ 08544, USA

${ }^{3}$ Department Chemistry, University of Kentucky, Lexington, KY 40506, USA

${ }^{4}$ Department of Chemical and Biological Engineering, Princeton University, Princeton, NJ 08544, USA

*These authors contributed equally

†Corresponding author:1loo@princeton.edu

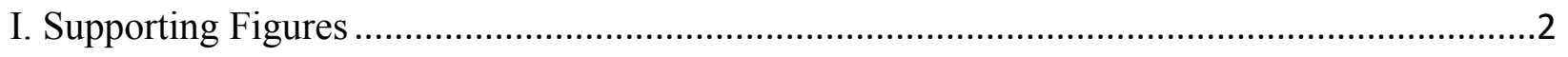

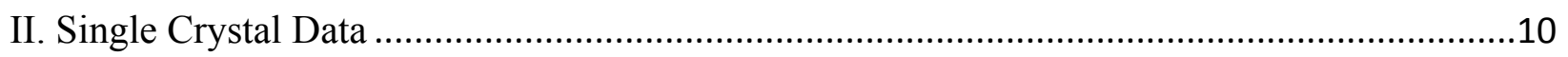

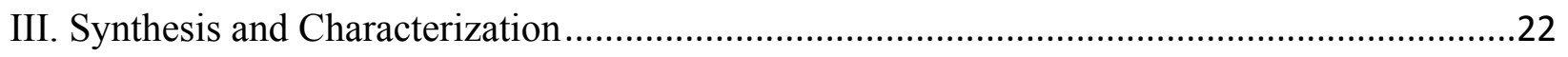

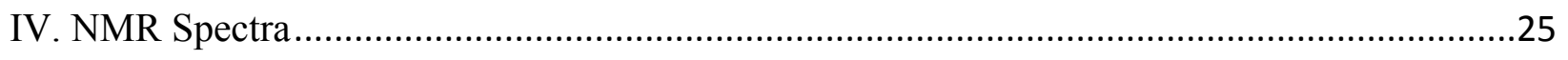

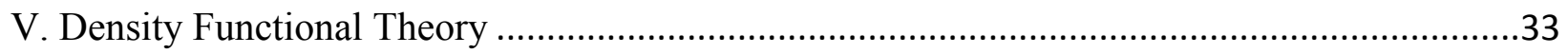

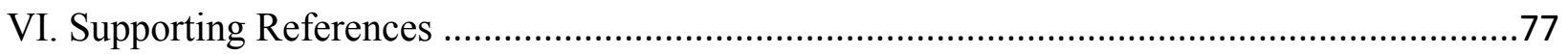




\section{Supporting Figures}
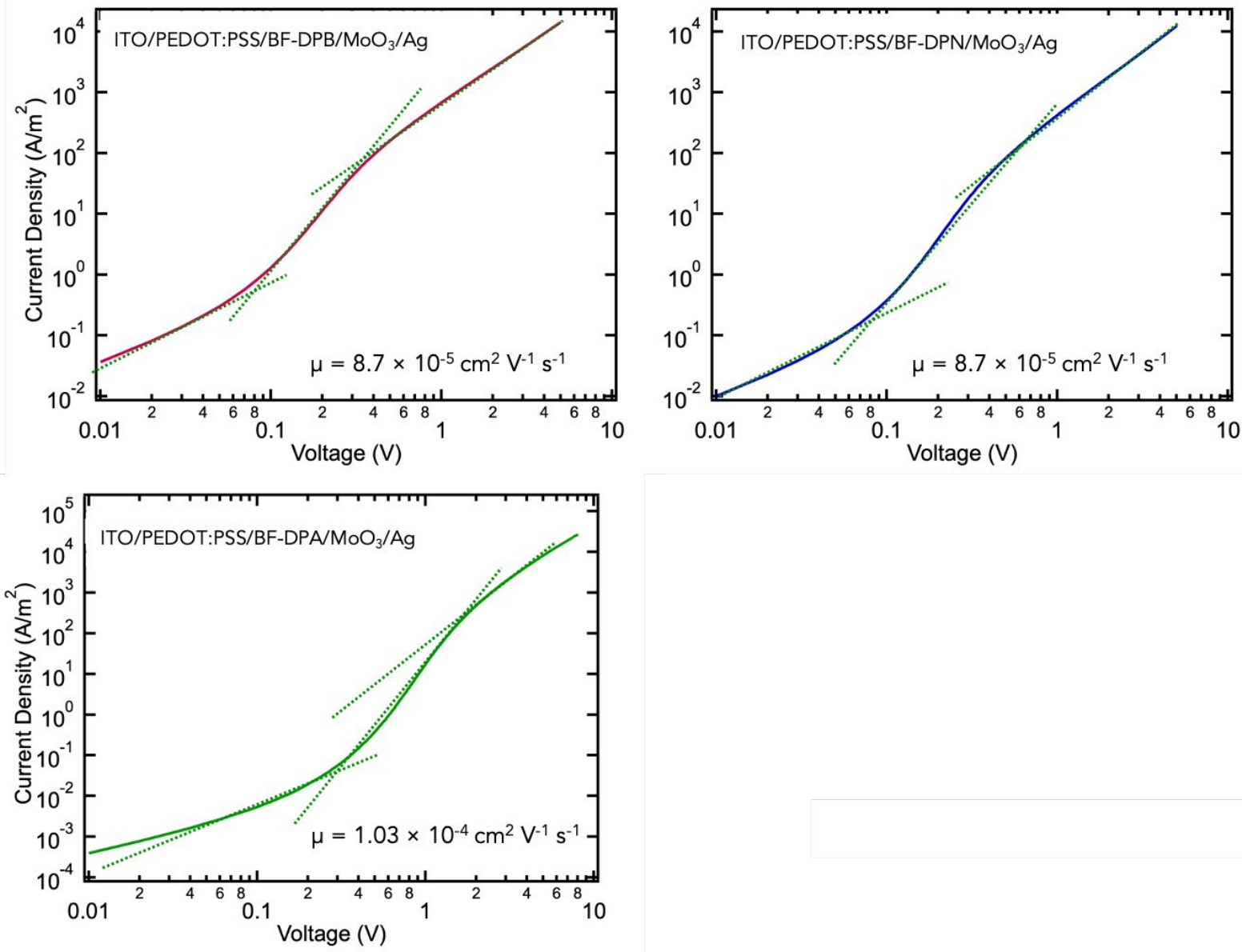

Supplemental Figure S1 |Hole mobility measurements by space charge limited current for BFDPB,BF-DPN, and BF-DPA. All hole mobilities are similar at $10^{-4} \mathrm{~cm}^{2} / \mathrm{V}$-s. Mobilities were calculated using Child's law, $\mathrm{J}=9 \varepsilon_{0} \varepsilon_{\mathrm{T}} \mu_{\mathrm{h}} \mathrm{V}^{2} / 8 \mathrm{~L}^{3}$, where $\mathrm{J}$ is the current density, $\mathrm{L}$ is the active thickness of the films, $\mu_{\mathrm{h}}$ is the hole mobility, $\varepsilon_{\mathrm{T}}$ is the relative dielectric constant of the transport medium and $\varepsilon_{\mathrm{T}}=3$ for this calculation, $\varepsilon_{0}$ is the permittivity of free space $\left(8.85 \times 10^{-12} \mathrm{~F} \mathrm{~m}^{-1}\right)$, $\mathrm{V}$ is the applied voltage to the device. All films were $80 \mathrm{~nm}$. 


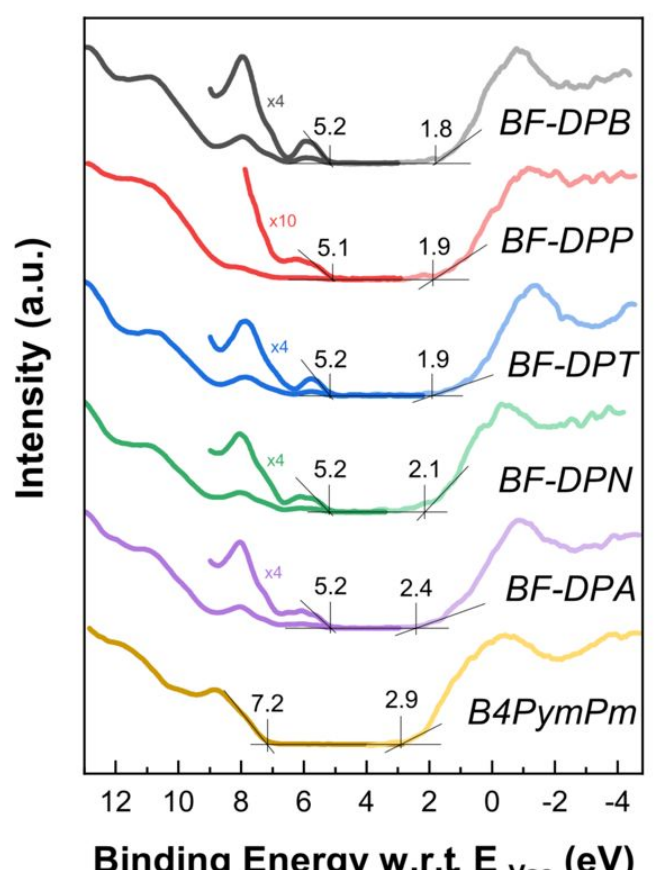

Supplemental Figure S2 | Ultraviolet photoelectron spectroscopy (UPS He I; left, darker color) and inverse photoelectron spectroscopy (IPES; right, lighter color) spectra of the donors and acceptor studied in this work. The binding energy is plotted with respect to vacuum level ( $\left.\mathrm{E}_{\mathrm{Vac}}\right)$ and the intensity of each spectra is normalized relative to the most intense feature in the UPS data, and, separately, in the IPES data. The ionization energy (IE) and electron affinity (EA) are extracted for each material and labeled in the figure for clarity. 

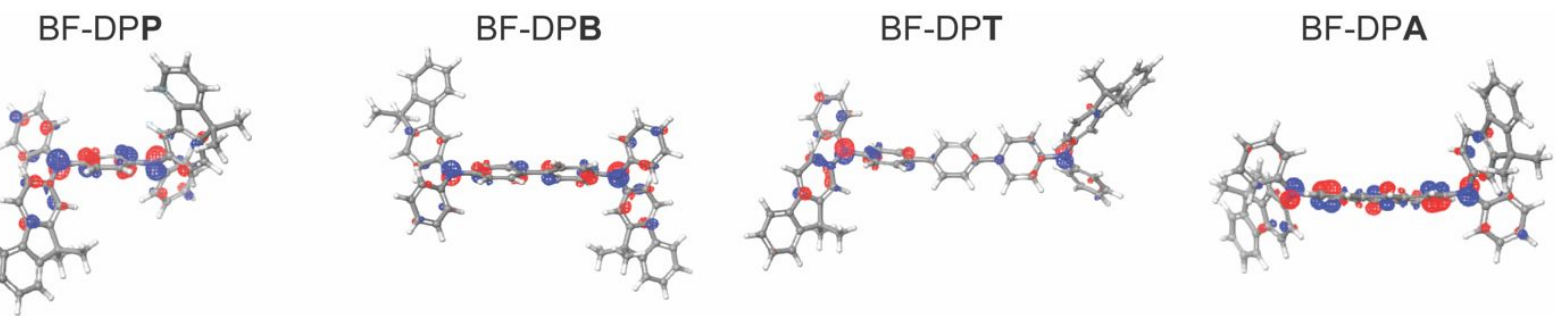

b
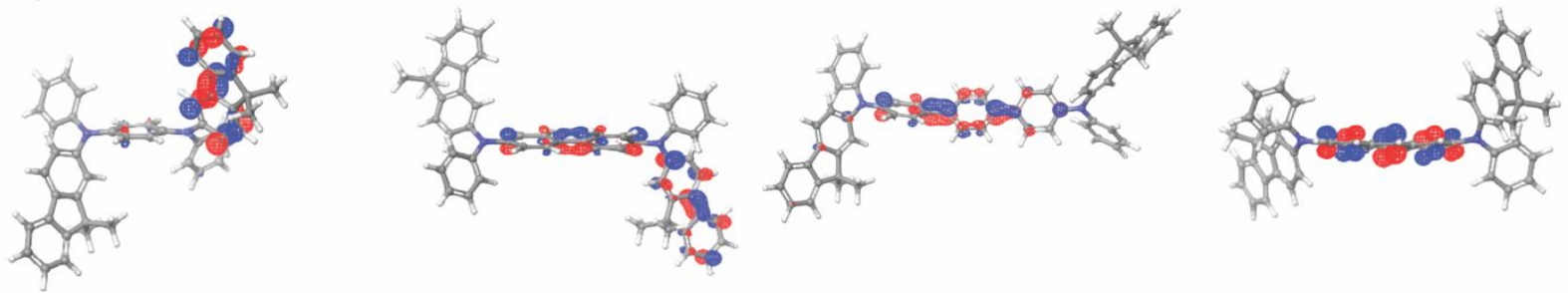

Supplemental Figure S3 $\mid$ a HOMO and b LUMO of BF-DPP, BF-DPP, BF-DPT, and BF-DPA generated by DFT. Level of theory $=$ B3LYP-D3, basis set $=6-31^{++} \mathrm{G}^{* *}$ and isovalue $=-0.05$. 
a

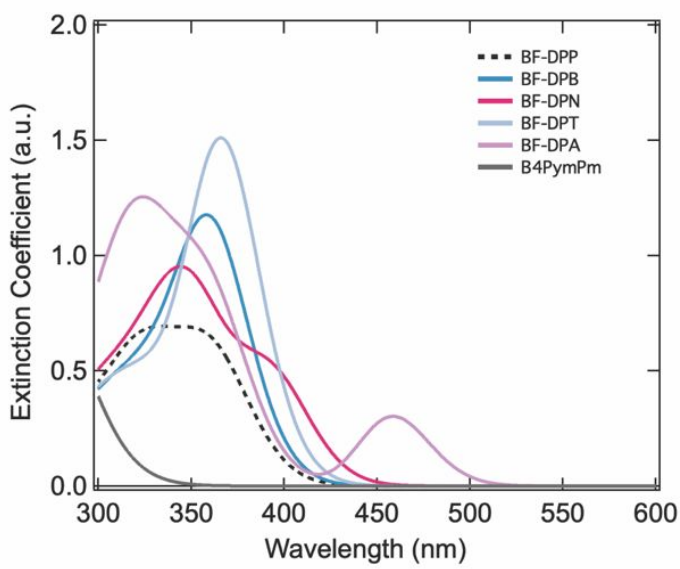

b

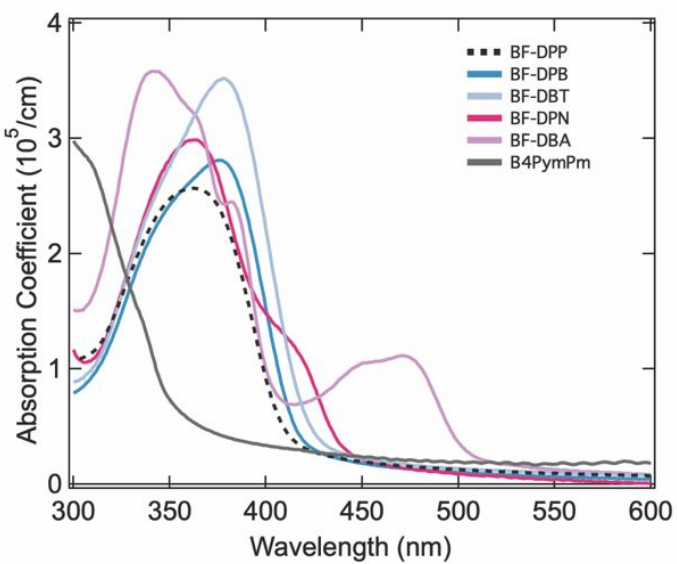

Supplemental Figure S4 | a Calculated absorption spectra for the five donor materials (BFDPP, BF-DPB, BF-DPN, BF-DPT and BF-DPA) and B4PymPm using TD-DFT. Level of theory $=\mathrm{B} 3 \mathrm{LYP}-\mathrm{D} 3$, basis set $=6-31^{++} \mathrm{G}^{* *} \mathbf{b}$ Measured absorption spectra of the same materials from a. 

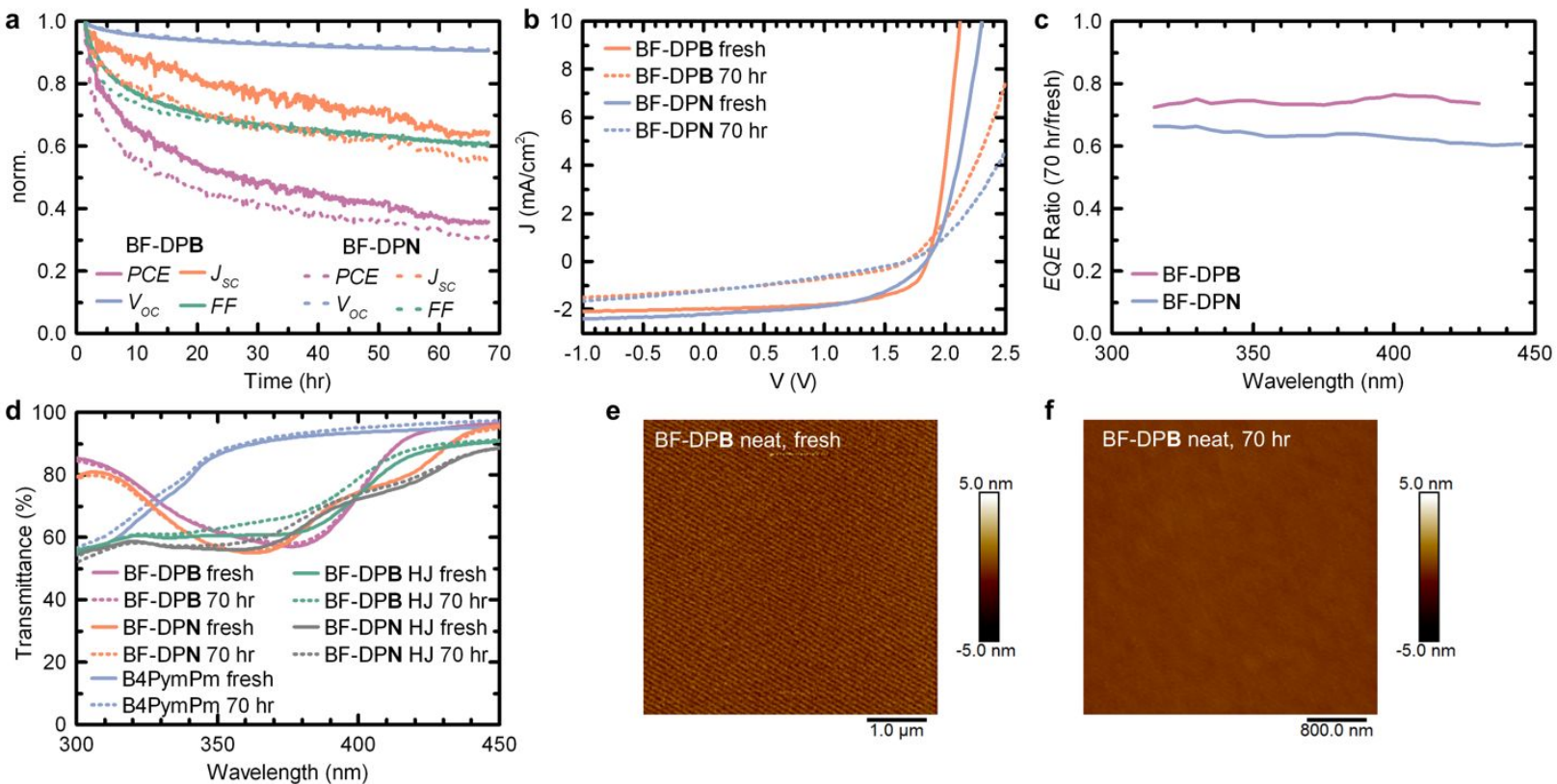

e
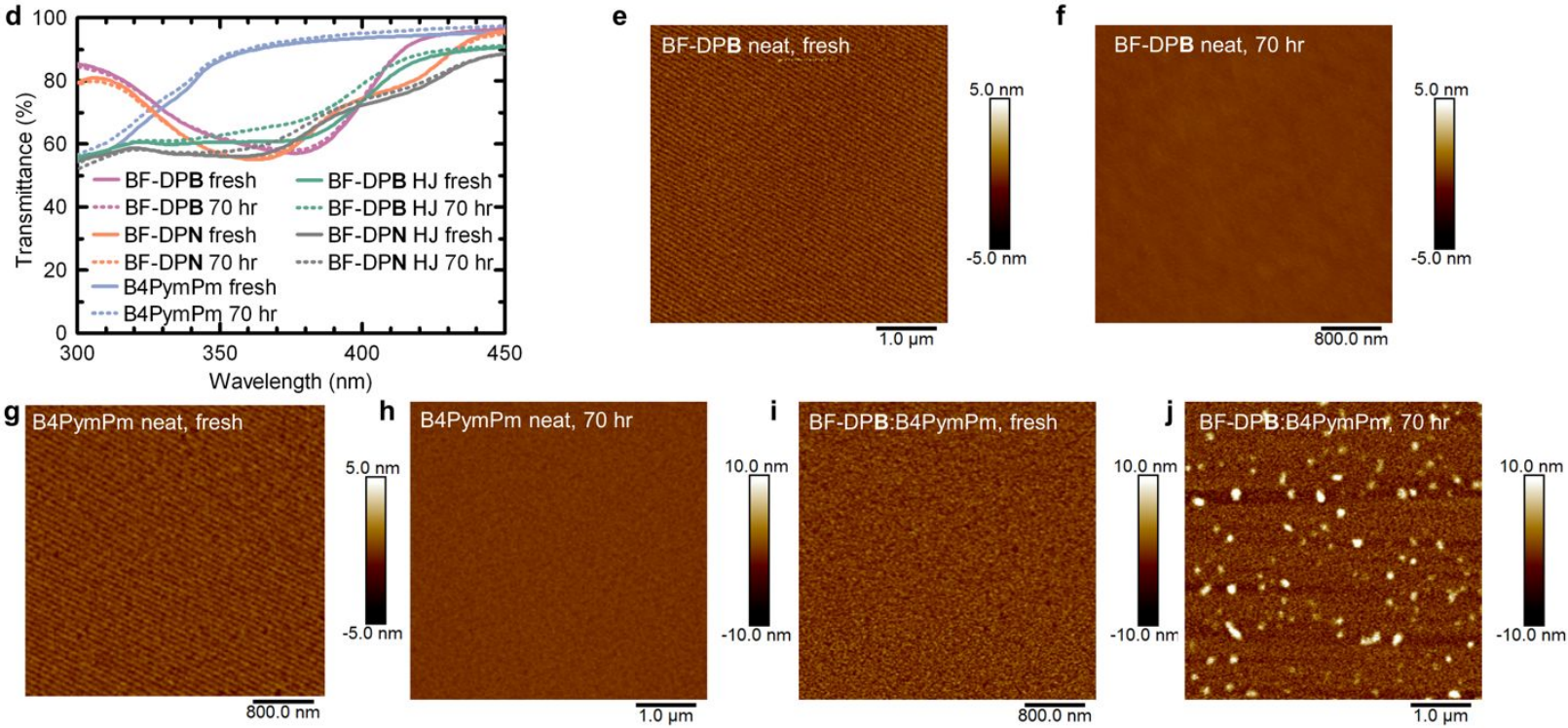

Supplemental Figure S5 | To quantify the operational stability of OPVs with NUV-absorbing active layers, we encapsulated opaque conventional OPVs incorporating either BF-DPB or BFDPN in a $\mathrm{N}_{2}$ glovebox with a glass cover slide and UV-curable epoxy seal around their periphery and exposed them to $70 \mathrm{hr}$ of simulated AM1.5G illumination from a metal halide lamp ( 2 suns intensity). The $J-V$ characteristics of these devices were measured continuously during aging, allowing us to track the time evolution of $J_{S C}, V_{O C}, F F$, and $P C E$ for both devices as shown in $\mathbf{a}$. We observe a rapid decrease in. $J_{S C}$ and $F F$ in both devices leading to $T_{80}$ lifetimes (i.e., the time it takes for $P C E$ to reach $80 \%$ of its starting value) of $<5 \mathrm{hr}$, and $T_{50}$ lifetimes $<24 \mathrm{hr}$ in both devices. The $J-V$ curves plotted before and after $70 \mathrm{hr}$ of aging in $\mathbf{b}$ show that the photocurrent becomes much more voltage-dependent, and the series resistance increases with time for both devices. The ratio of $E Q E$ spectra taken before and after $70 \mathrm{hr}$ of aging are effectively flat, as shown in $\mathbf{c}$, suggesting that charge extraction rather than charge generation becomes less effective with time - consistent with the increased photocurrent voltage-dependence in the $J-V$ curves. To better understand the origins of this efficiency loss, we aged 40-nm thick films of BF-DPB, BFDPN, B4PymPm and 80-nm thick films having blends of 1:1 BF-DPB:B4PymPm and BFDPN:B4PymPm on quartz substrates, and measured their transmittance spectra as a function of time, as shown in $\mathbf{d}$. While the transmittance spectra of the two donor films show effectively no change, the transmittance of the B4PymPm film changes slightly, and the HJ films with both donors change significantly. Surface topology measurements on neat films of BF-DPB, B4PymPm, and a BF-DPB:B4PymPm HJ grown on Si as measured by atomic force microscopy are shown before and after aging for $70 \mathrm{hr}$ in $\mathbf{e}-\mathbf{j}$. While the surface of the individual constituents remains smooth and featureless with time, the surface of the BF-DPB:B4PymPm HJ film 
significantly roughens with time, as shown in $\mathbf{j}$, consistent with phase segregation between the donor and acceptor materials or co-crystallization of the constituents, both hypothesis of which are under consideration. Thus, while the initial performance of these devices is promisingparticularly for transparent NUV-OPVs, as we will show-we conclude that additional molecular and device engineering of these materials systems is required to stabilize their morphology before they can be considered viable for practical applications.

Solar cells for lifetime testing were encapsulated in a $\mathrm{N}_{2}$ glovebox with a glass cover slide and UV-curable epoxy applied around the periphery such that only Al-coated ITO external contacts were exposed. Encapsulated devices were mounted onto printed circuit boards with $\mathrm{Ni}$ paste, Si paste, conductive $\mathrm{Cu}$ tape, and solder. Wires from the circuit boards were then electrically connected to a Keithley 2401 low voltage source measurement unit with a Keithley 2700 multimeter/data acquisition/switch system controlling two Keithley 7701 low voltage 32-channel differential multiplexer modules. The $J-V$ characteristics of each device were measured every 5 minutes, and their photovoltaic parameters: $J_{S C}, V_{O C}, F F$, and $P C E$ were extracted automatically as a function of time. Devices were continuously aged at their maximum power point with an active electrical load system from infinityPV ApS. Broad spectrum illumination was provided at $\sim 1200 \mathrm{~W} / \mathrm{m}^{2}$ from an ISOSun metal halide simulator from infinityPV ApS. 

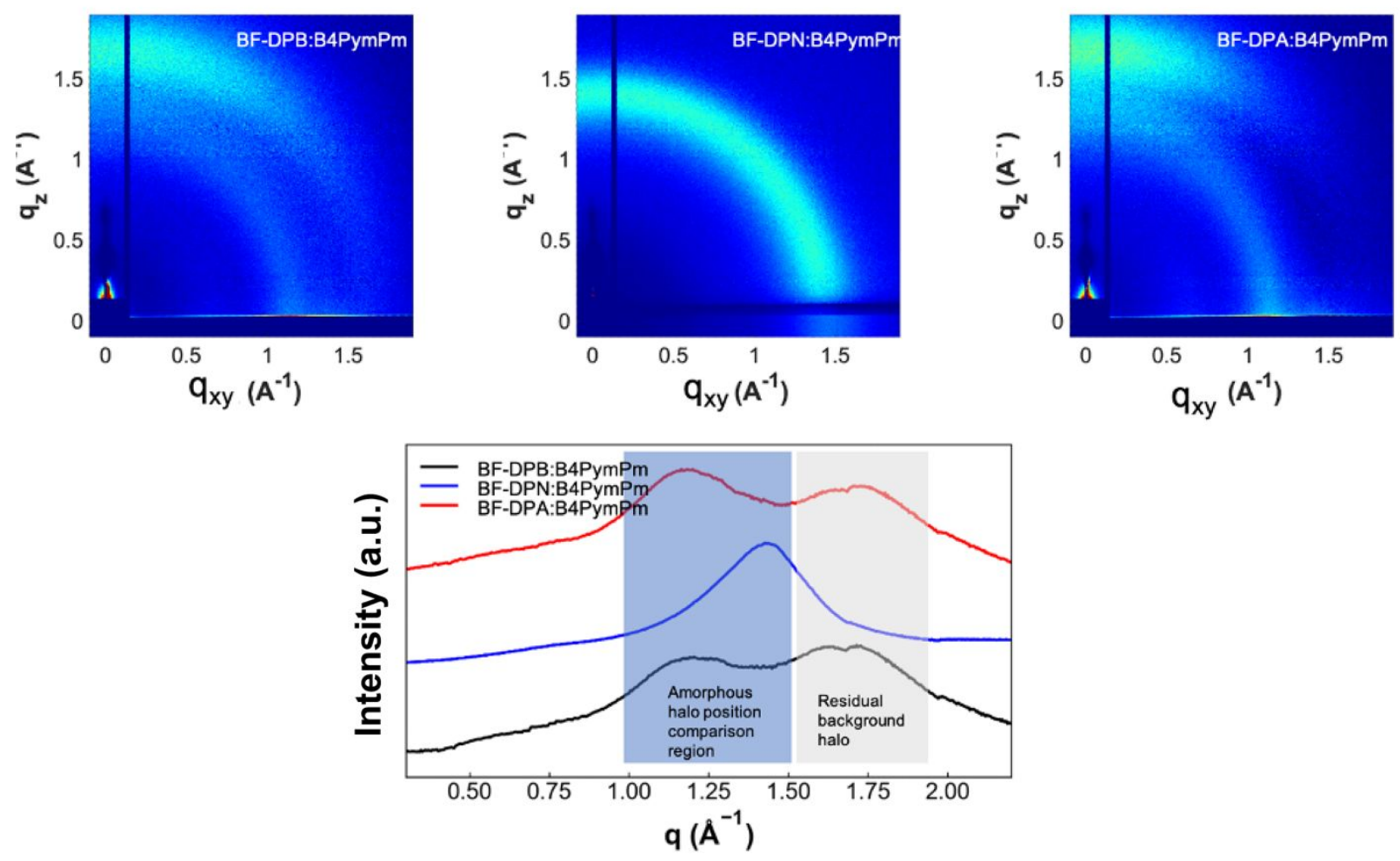

Supplemental Figure S6 |GIWAXS spectra for $80 \mathrm{~nm}$ blended heterojunction films aged under 1 -sun for three days in an inert nitrogen atmosphere. The films are largely amorphous with no crystalline features. 
Supplemental Table S1 | Thickness-dependent solar cell data.

\begin{tabular}{|c|c|c|c|c|c|c|}
\hline Device architecture & Donor molecule & HJ Thickness (nm) & $J_{s c}\left(\mathrm{~mA} / \mathrm{cm}^{2}\right)$ & $V_{O C}(\mathrm{~V})$ & $F F(\%)$ & PCE (\%) \\
\hline \multirow{17}{*}{$\begin{array}{l}\text { opaque, } \\
\text { conventional }\end{array}$} & \multirow{4}{*}{ BF-DPP } & 60 & 0.58 & 1.75 & 64 & 0.64 \\
\hline & & 80 & 0.56 & 1.74 & 66 & 0.64 \\
\hline & & 100 & 0.50 & 1.74 & 58 & 0.50 \\
\hline & & 120 & 0.55 & 1.74 & 48 & 0.49 \\
\hline & BF-DPB & 80 & 0.81 & 1.85 & 74 & 1.11 \\
\hline & \multirow{3}{*}{ BF-DPT } & 60 & 0.83 & 1.93 & 44 & 0.7 \\
\hline & & 70 & 0.83 & 1.93 & 46 & 0.74 \\
\hline & & 80 & 0.78 & 1.93 & 46 & 0.69 \\
\hline & \multirow{5}{*}{ BF-DPN } & 50 & 0.88 & 1.80 & 63 & 1.00 \\
\hline & & 60 & 0.99 & 1.81 & 65 & 1.16 \\
\hline & & 65 (different batch) & 0.97 & 1.84 & 67 & 1.20 \\
\hline & & 70 & 1.02 & 1.80 & 63 & 1.16 \\
\hline & & 80 & 1.01 & 1.81 & 61 & 1.12 \\
\hline & \multirow{4}{*}{ BF-DPA } & 60 & 1.22 & 1.74 & 39 & 0.81 \\
\hline & & 70 & 1.23 & 1.74 & 39 & 0.83 \\
\hline & & 80 & 1.22 & 1.74 & 40 & 0.85 \\
\hline & & 100 & 1.19 & 1.74 & 40 & 0.83 \\
\hline \multirow{12}{*}{ opaque, inverted } & \multirow{5}{*}{ BF-DPB } & 80 & 0.69 & 1.92 & 56 & 0.71 \\
\hline & & 100 & 0.68 & 1.91 & 50 & 0.67 \\
\hline & & 120 & 0.73 & 1.92 & 47 & 0.67 \\
\hline & & 120 (different batch) & 0.77 & 1.99 & 63 & 0.96 \\
\hline & & 140 & 0.82 & 1.92 & 49 & 0.78 \\
\hline & \multirow{4}{*}{ BF-DPT } & 70 & 0.49 & 2.04 & 46 & 0.46 \\
\hline & & 80 & 0.58 & 2.04 & 43 & 0.51 \\
\hline & & 90 & 0.62 & 2.03 & 40 & 0.50 \\
\hline & & 100 & 0.62 & 2.03 & 38 & 0.46 \\
\hline & \multirow{3}{*}{ BF-DPN } & 120 & 0.75 & 1.87 & 49 & 0.69 \\
\hline & & 150 & 0.79 & 1.86 & 42 & 0.62 \\
\hline & & 180 & 0.98 & 1.88 & 42 & 0.77 \\
\hline \multirow{12}{*}{$\begin{array}{l}\text { transparent, } \\
\text { inverted }\end{array}$} & \multirow{5}{*}{ BF-DPB } & 80 & 0.49 & 1.95 & 60 & 0.57 \\
\hline & & 100 & 0.55 & 1.95 & 56 & 0.60 \\
\hline & & 120 & 0.62 & 1.96 & 51 & 0.63 \\
\hline & & 120 (different batch) & 0.64 & 2.00 & 55 & 0.70 \\
\hline & & 140 & 0.63 & 1.96 & 49 & 0.6 \\
\hline & \multirow{4}{*}{ BF-DPT } & 70 & 0.43 & 2.02 & 37 & 0.33 \\
\hline & & 80 & 0.48 & 2.03 & 39 & 0.39 \\
\hline & & 90 & 0.51 & 2.03 & 40 & 0.41 \\
\hline & & 100 & 0.53 & 2.03 & 40 & 0.43 \\
\hline & \multirow{3}{*}{ BF-DPN } & 120 & 0.63 & 1.88 & 45 & 0.53 \\
\hline & & 150 & 0.69 & 1.88 & 45 & 0.58 \\
\hline & & 180 & 0.76 & 1.88 & 43 & 0.61 \\
\hline
\end{tabular}




\section{Single Crystal Data}
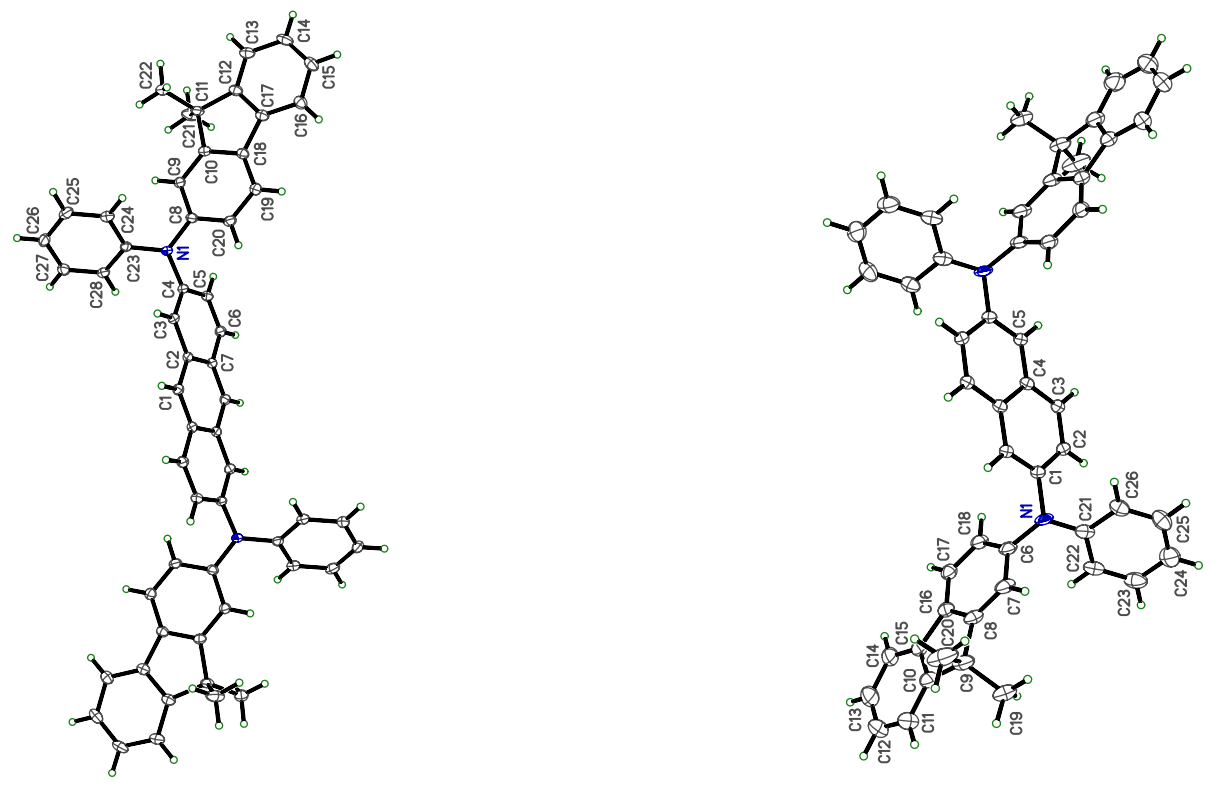

Supplemental Figure S7 |Single crystal structures for BF-DPA (left) and BF-DPN (right). X-ray diffraction data were collected at 90.0(2) K on a Bruker D8 Venture kappa-axis diffractometer using MoK(alpha) X-rays. Raw data were integrated, scaled, merged, and corrected for Lorentzpolarization effects using the APEX3 package. ${ }^{1}$ Corrections for absorption were applied using SADABS. ${ }^{2}$ The structures were solved by iterative dual-space methods ${ }^{3}$ and refinement was carried out against $\mathrm{F}^{\wedge} 2$ by weighted full-matrix least-squares. ${ }^{4}$ Hydrogen atoms were found in difference maps, but subsequently placed at calculated positions and refined using riding models. Non-hydrogen atoms were refined with anisotropic displacement parameters. Atomic scattering factors were taken from the International Tables for Crystallography. ${ }^{5}$ Crystal data and relevant details of the structure determinations are summarized in Table S2 for BF-DPN and Table S4 for BF-DPA, and selected geometrical parameters are given in Tables S3 for BF-DPN and Table S5 for BF-DPA.

Supplemental Table S2 | Crystal Data and Structure refinement for BF-DPN.

Identification code

Empirical formula

Formula weight

Temperature

Wavelength

Crystal system, space group $\mathrm{m} 21021$

C52 H42 N2

694.87

90.0(2) K

$0.71073 \mathrm{~A}$

Monoclinic, P2(1)/n 


$\begin{array}{ll}\text { Unit cell dimensions } & \mathrm{a}=12.3886(5) \mathrm{A} \\ & \mathrm{b}=13.0511(6) \mathrm{A} \\ & \mathrm{c}=12.9348(6) \mathrm{A} \\ & 1859.22(14) \mathrm{A}^{\wedge} 3 \\ \text { Volume } & 2,1.241 \mathrm{Mg} / \mathrm{m}^{\wedge} 3 \\ \text { Z, Calculated density } & 0.071 \mathrm{~mm}^{\wedge}-1 \\ \text { Absorption coefficient } & 736 \\ \mathrm{~F}(000) & 0.250 \times 0.220 \times 0.200 \mathrm{~mm} \\ \text { Crystal size } & 2.361 \text { to } 27.528 \text { deg. } \\ \text { Theta range for data } & -16<=\mathrm{h}<=16,-16<=\mathrm{k}<=16,- \\ \text { collection } & 16<=1<=16 \\ \text { Limiting indices } & 27652 / 4271 \text { [R(int })=0.0537] \\ \text { Reflections collected / unique } & \\ \text { Completeness to theta }= & 100.00 \% \\ \text { 25.242 } & \text { Semi-empirical for equivalents } \\ \text { Absorption correction } & 0.971 \text { and } 0.681 \\ \text { Max. and min. transmission } & \text { Full-matrix least-squares on } \mathrm{F}^{\wedge} 2 \\ \text { Refinement method } & 4271 / 194 / 290 \\ \text { Data / restraints / parameters } & 1.072 \\ \text { Goodness-of-fit on } \mathrm{F}^{\wedge} 2 & \mathrm{R} 1=0.0527, \mathrm{wR} 2=0.1259 \\ \text { Final R indices [I }>2 \text { sigma(I) } & \mathrm{R} 1=0.0614, \mathrm{wR} 2=0.1314 \\ \text { R indices (all data) } & 0.0058(13) \\ \text { Extinction coefficient } & 0.517 \text { and }-0.406 \text { e. A^-3 } \\ \text { Largest diff. peak and hole } & \end{array}$

alpha $=90$ deg. beta $=117.252(1)$ deg. gamma $=90 \mathrm{deg}$. 
Supplemental Table S3 | Bond lengths ( $\AA$ ) and angles (deg) for BF-DPN.

\begin{tabular}{|c|c|}
\hline N1-C21 & $1.325(6)$ \\
\hline N1-C6 & $1.440(6)$ \\
\hline N1-C1 & $1.481(7)$ \\
\hline C1-C5\#1 & $1.358(4)$ \\
\hline C1-C2 & $1.442(4)$ \\
\hline $\mathrm{C} 2-\mathrm{C} 3$ & $1.373(3)$ \\
\hline $\mathrm{C} 2-\mathrm{H} 2$ & 0.95 \\
\hline $\mathrm{C} 3-\mathrm{C} 4$ & $1.411(4)$ \\
\hline C3-H3 & 0.95 \\
\hline C4-C5 & $1.422(4)$ \\
\hline C4-C4\#1 & $1.435(8)$ \\
\hline C5-H5 & 0.95 \\
\hline N1'-C1' & $1.382(8)$ \\
\hline N1'-C6 & $1.417(6)$ \\
\hline N1'-C21 & $1.486(7)$ \\
\hline C1'-C2' & $1.394(5)$ \\
\hline C1'-C5'\#1 & $1.396(5)$ \\
\hline C2'-C3' & $1.418(5)$ \\
\hline $\mathrm{C} 2^{\prime}-\mathrm{H} 2^{\prime}$ & 0.95 \\
\hline C3'-C3'\#1 & $1.415(9)$ \\
\hline C3'-C4' & $1.418(5)$ \\
\hline C4'-C5' & $1.375(4)$ \\
\hline C4'-H4' & 0.95 \\
\hline C5'-H5' & 0.95 \\
\hline C6-C7 & $1.395(2)$ \\
\hline C6-C18 & $1.400(2)$ \\
\hline C7-C8 & $1.383(2)$ \\
\hline C7-H7 & 0.95 \\
\hline C8-C16 & $1.405(2)$ \\
\hline C8-C9 & $1.526(2)$ \\
\hline C9-C10 & $1.522(3)$ \\
\hline C9-C19 & $1.538(2)$ \\
\hline C9-C20 & $1.543(3)$ \\
\hline C10-C11 & $1.386(2)$ \\
\hline C10-C15 & $1.401(3)$ \\
\hline $\mathrm{C} 11-\mathrm{C} 12$ & $1.380(4)$ \\
\hline C11-H11 & 0.95 \\
\hline C12-C13 & $1.383(4)$ \\
\hline C12-H12 & 0.95 \\
\hline
\end{tabular}




\begin{tabular}{|c|c|}
\hline C13-C14 & $1.394(3)$ \\
\hline C13-H13 & 0.95 \\
\hline C14-C15 & $1.391(3)$ \\
\hline C14-H14 & 0.95 \\
\hline C15-C16 & $1.469(2)$ \\
\hline C16-C17 & $1.390(2)$ \\
\hline C17-C18 & $1.391(2)$ \\
\hline C17-H17 & 0.95 \\
\hline C18-H18 & 0.95 \\
\hline C19-H19A & 0.98 \\
\hline C19-H19B & 0.98 \\
\hline C19-H19C & 0.98 \\
\hline C20-H20A & 0.98 \\
\hline C20-H20B & 0.98 \\
\hline C20-H20C & 0.98 \\
\hline C21-C26 & $1.396(2)$ \\
\hline $\mathrm{C} 21-\mathrm{C} 22$ & $1.401(2)$ \\
\hline $\mathrm{C} 22-\mathrm{C} 23$ & $1.386(2)$ \\
\hline $\mathrm{C} 22-\mathrm{H} 22$ & 0.95 \\
\hline $\mathrm{C} 23-\mathrm{C} 24$ & $1.385(3)$ \\
\hline $\mathrm{C} 23-\mathrm{H} 23$ & 0.95 \\
\hline $\mathrm{C} 24-\mathrm{C} 25$ & $1.387(3)$ \\
\hline C24-H24 & 0.95 \\
\hline $\mathrm{C} 25-\mathrm{C} 26$ & $1.380(2)$ \\
\hline $\mathrm{C} 25-\mathrm{H} 25$ & 0.95 \\
\hline C26-H26 & 0.95 \\
\hline C21-N1-C6 & $124.0(6)$ \\
\hline C21-N1-C1 & $113.8(4)$ \\
\hline C6-N1-C1 & $120.8(4)$ \\
\hline C5\#1-C1-C2 & $119.8(3)$ \\
\hline C5\#1-C1-N1 & $120.0(3)$ \\
\hline $\mathrm{C} 2-\mathrm{C} 1-\mathrm{N} 1$ & $120.2(3)$ \\
\hline $\mathrm{C} 3-\mathrm{C} 2-\mathrm{C} 1$ & $120.1(3)$ \\
\hline $\mathrm{C} 3-\mathrm{C} 2-\mathrm{H} 2$ & 120 \\
\hline $\mathrm{C} 1-\mathrm{C} 2-\mathrm{H} 2$ & 120 \\
\hline $\mathrm{C} 2-\mathrm{C} 3-\mathrm{C} 4$ & $121.0(3)$ \\
\hline $\mathrm{C} 2-\mathrm{C} 3-\mathrm{H} 3$ & 119.5 \\
\hline C4-C3-H3 & 119.5 \\
\hline C3-C4-C5 & $122.2(3)$ \\
\hline
\end{tabular}




\begin{tabular}{|c|c|}
\hline C3-C4-C4\#1 & $118.8(4)$ \\
\hline C5-C4-C4\#1 & $119.0(4)$ \\
\hline $\mathrm{C} 1 \# 1-\mathrm{C} 5-\mathrm{C} 4$ & $121.2(3)$ \\
\hline C1\#1-C5-H5 & 119.4 \\
\hline C4-C5-H5 & 119.4 \\
\hline C1'-N1'-C6 & $115.0(4)$ \\
\hline C1'-N1'-C21 & $129.6(5)$ \\
\hline C6-N1'-C21 & $114.6(6)$ \\
\hline $\mathrm{N} 1{ }^{\prime}-\mathrm{C} 1{ }^{\prime}-\mathrm{C} 2{ }^{\prime}$ & $115.8(3)$ \\
\hline N1'-C1'-C5'\#1 & $123.9(4)$ \\
\hline $\mathrm{C} 2^{\prime}-\mathrm{C} 1^{\prime}-\mathrm{C} 5^{\prime} \# 1$ & $120.3(3)$ \\
\hline C1'-C2'-C3' & $119.8(4)$ \\
\hline C1'-C2'-H2' & 120.1 \\
\hline C3'-C2'-H2' & 120.1 \\
\hline C3'\#1-C3'-C2' & $119.8(5)$ \\
\hline C3'\#1-C3'-C4' & $118.6(4)$ \\
\hline C2'-C3'-C4' & $121.6(4)$ \\
\hline C5'-C4'-C3' & $120.9(3)$ \\
\hline C5'-C4'-H4' & 119.5 \\
\hline C3'-C4'-H4' & 119.5 \\
\hline C4'-C5'-H5' & 119.7 \\
\hline C1'\#1-C5'-H5' & 119.7 \\
\hline C7-C6-C18 & $120.21(14)$ \\
\hline C7-C6-N1' & $120.8(2)$ \\
\hline C18-C6-N1' & $119.0(2)$ \\
\hline C7-C6-N1 & $120.8(2)$ \\
\hline C18-C6-N1 & $118.4(2)$ \\
\hline C8-C7-C6 & $119.32(14)$ \\
\hline C8-C7-H7 & 120.3 \\
\hline C6-C7-H7 & 120.3 \\
\hline C7-C8-C16 & $120.42(14)$ \\
\hline C7-C8-C9 & $128.63(15)$ \\
\hline C16-C8-C9 & $110.95(14)$ \\
\hline C10-C9-C8 & $100.94(13)$ \\
\hline C10-C9-C19 & $113.09(13)$ \\
\hline C8-C9-C19 & $112.75(14)$ \\
\hline C10-C9-C20 & $110.23(16)$ \\
\hline C8-C9-C20 & $110.61(13)$ \\
\hline C19-C9-C20 & $109.04(15)$ \\
\hline C11-C10-C15 & $119.88(19)$ \\
\hline
\end{tabular}




\begin{tabular}{|c|c|}
\hline C11-C10-C9 & $128.87(19)$ \\
\hline C15-C10-C9 & $111.25(14)$ \\
\hline C12-C11-C10 & $119.1(2)$ \\
\hline C12-C11-H11 & 120.4 \\
\hline C10-C11-H11 & 120.4 \\
\hline C11-C12-C13 & $121.10(18)$ \\
\hline C11-C12-H12 & 119.5 \\
\hline C13-C12-H12 & 119.5 \\
\hline C12-C13-C14 & $120.8(2)$ \\
\hline C12-C13-H13 & 119.6 \\
\hline C14-C13-H13 & 119.6 \\
\hline C15-C14-C13 & $118.0(2)$ \\
\hline C15-C14-H14 & 121 \\
\hline C13-C14-H14 & 121 \\
\hline C14-C15-C10 & $121.07(16)$ \\
\hline C14-C15-C16 & $130.56(16)$ \\
\hline C10-C15-C16 & $108.35(15)$ \\
\hline C17-C16-C8 & $120.44(14)$ \\
\hline C17-C16-C15 & $131.24(15)$ \\
\hline C8-C16-C15 & $108.28(14)$ \\
\hline C16-C17-C18 & $119.02(14)$ \\
\hline C16-C17-H17 & 120.5 \\
\hline C18-C17-H17 & 120.5 \\
\hline C17-C18-C6 & $120.55(14)$ \\
\hline C17-C18-H18 & 119.7 \\
\hline C6-C18-H18 & 119.7 \\
\hline C9-C19-H19A & 109.5 \\
\hline C9-C19-H19B & 109.5 \\
\hline H19A-C19-H19B & 109.5 \\
\hline C9-C19-H19C & 109.5 \\
\hline H19A-C19-H19C & 109.5 \\
\hline H19B-C19-H19C & 109.5 \\
\hline C9-C20-H20A & 109.5 \\
\hline C9-C20-H20B & 109.5 \\
\hline H20A-C20-H20B & 109.5 \\
\hline C9-C20-H20C & 109.5 \\
\hline $\mathrm{H} 20 \mathrm{~A}-\mathrm{C} 20-\mathrm{H} 20 \mathrm{C}$ & 109.5 \\
\hline H20B-C20-H20C & 109.5 \\
\hline N1-C21-C26 & $123.0(3)$ \\
\hline N1-C21-C22 & $118.5(3)$ \\
\hline
\end{tabular}


C26-C21-C22

C26-C21-N1'

C22-C21-N1'

$\mathrm{C} 23-\mathrm{C} 22-\mathrm{C} 21$

C23-C22-H22

$\mathrm{C} 21-\mathrm{C} 22-\mathrm{H} 22$

C24-C23-C22

$\mathrm{C} 24-\mathrm{C} 23-\mathrm{H} 23$

C22-C23-H 23

C23-C24-C25

C23-C24-H24

C25-C24-H24

C26-C25-C24

C26-C25-H25

C24-C25-H25

C25-C26-C21

$\mathrm{C} 25-\mathrm{C} 26-\mathrm{H} 26$
118.34(15)

119.1(3)

122.6(3)

120.27(15)

119.9

119.9

120.95(16)

119.5

119.5

$118.86(17)$

120.6

120.6

120.82(16)

119.6

119.6

120.74(15)

119.6

119.6

Symmetry transformations used to generate equivalent atoms: $\# 1-\mathrm{x}+1,-\mathrm{y}+1,-\mathrm{z}+1$

Supplemental Table S4 | Crystal Data and Structure refinement for BF-DPA.

Identification code

Empirical formula

Formula weight

Temperature

Wavelength

Crystal system, space group

Unit cell dimensions

Volume

$Z$, Calculated density

Absorption coefficient

$\mathrm{F}(000)$

Crystal size

Theta range for data

collection

Limiting indices $\mathrm{m} 21015$

C56 H44 N2

744.93

$90.0(2) \mathrm{K}$

$0.71073 \mathrm{~A}$

Triclinic, P-1

$\mathrm{a}=8.9703(2) \mathrm{A}$

$\mathrm{b}=10.4841(3) \mathrm{A}$

$\mathrm{c}=11.6663(2) \mathrm{A}$

1001.83(4) $\mathrm{A}^{\wedge} 3$

$1,1.235 \mathrm{Mg} / \mathrm{m}^{\wedge} 3$

$0.071 \mathrm{~mm}^{\wedge}-1$

394

$0.260 \times 0.220 \times 0.180 \mathrm{~mm}$

2.117 to $27.505 \mathrm{deg}$. $-11<=\mathrm{h}<=11,-13<=\mathrm{k}<=13$, $14<=1<=15$ alpha $=107.199(1)$

deg.

beta $=95.614(1) \mathrm{deg}$.

gamma $=103.637(1)$

deg. 
Reflections collected /

unique

$64448 / 4586[\mathrm{R}(\mathrm{int})=0.0590]$

Completeness to theta $=$

25.242

$99.80 \%$

Absorption correction

Semi-empirical from equivalents

Max. and min. transmission

0.971 and 0.879

Refinement method

Full-matrix least-squares on $\mathrm{F}^{\wedge} 2$

Data / restraints / parameters

Goodness-of-fit on $\mathrm{F}^{\wedge} 2$

4586 / 0 / 264

Final $\mathrm{R}$ indices

[I $>2 \operatorname{sigma}(\mathrm{I})]$

1.067

$\mathrm{R}$ indices (all data)

$\mathrm{R} 1=0.0334$

$\mathrm{wR} 2=0.0861$

Extinction coefficient

$\mathrm{R} 1=0.0406$

$\mathrm{wR} 2=0.0898$

Largest diff. peak and hole

$\mathrm{n} / \mathrm{a}$

0.206 and -0.192 e. $\mathrm{A}^{\wedge}-3$

Supplemental Table S5 | Bond lengths $(\AA)$ and angles (deg) for BF-DPA.

$\begin{array}{ll}\mathrm{N} 1-\mathrm{C} 8 & 1.4122(11) \\ \mathrm{N} 1-\mathrm{C} 23 & 1.4154(11) \\ \mathrm{N} 1-\mathrm{C} 4 & 1.4279(11) \\ \mathrm{C} 1-\mathrm{C} 7 \# 1 & 1.3962(12) \\ \mathrm{C} 1-\mathrm{C} 2 & 1.3984(12) \\ \mathrm{C} 1-\mathrm{H} 1 & 0.95 \\ \mathrm{C} 2-\mathrm{C} 3 & 1.4283(12) \\ \mathrm{C} 2-\mathrm{C} 7 & 1.4365(12) \\ \mathrm{C} 3-\mathrm{C} 4 & 1.3649(13) \\ \mathrm{C} 3-\mathrm{H} 3 & 0.95 \\ \mathrm{C} 4-\mathrm{C} 5 & 1.4315(13) \\ \mathrm{C} 5-\mathrm{C} 6 & 1.3616(12) \\ \mathrm{C} 5-\mathrm{H} 5 & 0.95 \\ \mathrm{C} 6-\mathrm{C} 7 & 1.4303(12) \\ \mathrm{C} 6-\mathrm{H} 6 & 0.95 \\ \mathrm{C} 8-\mathrm{C} 20 & 1.3987(13) \\ \mathrm{C} 8-\mathrm{C} 9 & 1.4030(12) \\ \mathrm{C} 9-\mathrm{C} 10 & 1.3815(13) \\ \mathrm{C} 9-\mathrm{H} 9 & 0.95 \\ \mathrm{C} 10-\mathrm{C} 18 & 1.4028(13) \\ \mathrm{C} 10-\mathrm{C} 11 & 1.5212(12) \\ \mathrm{C} 11-\mathrm{C} 12 & 1.5235(13) \\ \mathrm{C} 11-\mathrm{C} 22 & 1.5361(14) \\ \mathrm{C} 11-\mathrm{C} 21 & 1.5386(13) \\ \mathrm{C} 12-\mathrm{C} 13 & 1.3858(13)\end{array}$




\begin{tabular}{|c|c|}
\hline C12-C17 & $1.4039(13)$ \\
\hline C13-C14 & $1.3938(14)$ \\
\hline C13-H13 & 0.95 \\
\hline C14-C15 & $1.3898(15)$ \\
\hline C14-H14 & 0.95 \\
\hline C15-C16 & $1.3899(14)$ \\
\hline C15-H15 & 0.95 \\
\hline C16-C17 & $1.3911(13)$ \\
\hline C16-H16 & 0.95 \\
\hline C17-C18 & $1.4675(12)$ \\
\hline C18-C19 & $1.3880(13)$ \\
\hline C19-C20 & $1.3893(13)$ \\
\hline C19-H19 & 0.95 \\
\hline C20-H20 & 0.95 \\
\hline C21-H21A & 0.98 \\
\hline C21-H21B & 0.98 \\
\hline $\mathrm{C} 21-\mathrm{H} 21 \mathrm{C}$ & 0.98 \\
\hline C22-H22A & 0.98 \\
\hline C22-H22B & 0.98 \\
\hline $\mathrm{C} 22-\mathrm{H} 22 \mathrm{C}$ & 0.98 \\
\hline C23-C28 & $1.3937(13)$ \\
\hline C23-C24 & $1.3981(12)$ \\
\hline $\mathrm{C} 24-\mathrm{C} 25$ & $1.3883(13)$ \\
\hline C24-H24 & 0.95 \\
\hline $\mathrm{C} 25-\mathrm{C} 26$ & $1.3871(15)$ \\
\hline $\mathrm{C} 25-\mathrm{H} 25$ & 0.95 \\
\hline C26-C27 & $1.3869(14)$ \\
\hline C26-H26 & 0.95 \\
\hline C27-C28 & $1.3827(13)$ \\
\hline C27-H27 & 0.95 \\
\hline C28-H28 & 0.95 \\
\hline C8-N1-C23 & $121.76(7)$ \\
\hline C8-N1-C4 & $119.57(7)$ \\
\hline C23-N1-C4 & $118.62(7)$ \\
\hline $\mathrm{C} 7 \# 1-\mathrm{C} 1-\mathrm{C} 2$ & $121.59(8)$ \\
\hline C7\#1-C1-H1 & 119.2 \\
\hline C2-C1-H1 & 119.2 \\
\hline $\mathrm{C} 1-\mathrm{C} 2-\mathrm{C} 3$ & $121.95(8)$ \\
\hline $\mathrm{C} 1-\mathrm{C} 2-\mathrm{C} 7$ & $119.14(8)$ \\
\hline
\end{tabular}




\begin{tabular}{|c|c|}
\hline $\mathrm{C} 3-\mathrm{C} 2-\mathrm{C} 7$ & 118.91(8) \\
\hline $\mathrm{C} 4-\mathrm{C} 3-\mathrm{C} 2$ & $121.01(8)$ \\
\hline C4-C3-H3 & 119.5 \\
\hline $\mathrm{C} 2-\mathrm{C} 3-\mathrm{H} 3$ & 119.5 \\
\hline C3-C4-N1 & $120.63(8)$ \\
\hline $\mathrm{C} 3-\mathrm{C} 4-\mathrm{C} 5$ & $120.20(8)$ \\
\hline N1-C4-C5 & $119.10(8)$ \\
\hline C6-C5-C4 & $120.23(8)$ \\
\hline C6-C5-H5 & 119.9 \\
\hline C4-C5-H5 & 119.9 \\
\hline C5-C6-C7 & $121.40(8)$ \\
\hline C5-C6-H6 & 119.3 \\
\hline C7-C6-H6 & 119.3 \\
\hline $\mathrm{C} 1 \# 1-\mathrm{C} 7-\mathrm{C} 6$ & $122.50(8)$ \\
\hline $\mathrm{C} 1 \# 1-\mathrm{C} 7-\mathrm{C} 2$ & $119.26(8)$ \\
\hline C6-C7-C2 & $118.23(8)$ \\
\hline C20-C8-C9 & $119.55(8)$ \\
\hline C20-C8-N1 & $119.56(8)$ \\
\hline C9-C8-N1 & $120.88(8)$ \\
\hline C10-C9-C8 & $119.15(8)$ \\
\hline C10-C9-H9 & 120.4 \\
\hline C8-C9-H9 & 120.4 \\
\hline C9-C10-C18 & $121.09(8)$ \\
\hline C9-C10-C11 & $127.83(8)$ \\
\hline C18-C10-C11 & $111.08(8)$ \\
\hline C10-C11-C12 & $101.22(7)$ \\
\hline C10-C11-C22 & $111.22(8)$ \\
\hline C12-C11-C22 & $112.44(8)$ \\
\hline C10-C11-C21 & $111.06(7)$ \\
\hline C12-C11-C21 & $110.96(8)$ \\
\hline C22-C11-C21 & $109.73(8)$ \\
\hline C13-C12-C17 & $120.34(9)$ \\
\hline C13-C12-C11 & $128.71(9)$ \\
\hline C17-C12-C11 & $110.91(8)$ \\
\hline C12-C13-C14 & $118.84(9)$ \\
\hline C12-C13-H13 & 120.6 \\
\hline C14-C13-H13 & 120.6 \\
\hline C15-C14-C13 & $120.72(9)$ \\
\hline C15-C14-H14 & 119.6 \\
\hline C13-C14-H14 & 119.6 \\
\hline
\end{tabular}




\begin{tabular}{|c|c|}
\hline C16-C15-C14 & $120.83(9)$ \\
\hline C16-C15-H15 & 119.6 \\
\hline C14-C15-H15 & 119.6 \\
\hline C15-C16-C17 & $118.56(9)$ \\
\hline C15-C16-H16 & 120.7 \\
\hline C17-C16-H16 & 120.7 \\
\hline C16-C17-C12 & $120.69(9)$ \\
\hline C16-C17-C18 & $130.88(9)$ \\
\hline C12-C17-C18 & $108.43(8)$ \\
\hline C19-C18-C10 & $119.87(8)$ \\
\hline C19-C18-C17 & $131.78(9)$ \\
\hline C10-C18-C17 & $108.36(8)$ \\
\hline C18-C19-C20 & $119.27(9)$ \\
\hline C18-C19-H19 & 120.4 \\
\hline C20-C19-H19 & 120.4 \\
\hline C19-C20-C8 & $121.05(8)$ \\
\hline C19-C20-H20 & 119.5 \\
\hline C8-C20-H20 & 119.5 \\
\hline C11-C21-H21A & 109.5 \\
\hline C11-C21-H21B & 109.5 \\
\hline $\mathrm{H} 21 \mathrm{~A}-\mathrm{C} 21-\mathrm{H} 21 \mathrm{~B}$ & 109.5 \\
\hline C11-C21-H21C & 109.5 \\
\hline $\mathrm{H} 21 \mathrm{~A}-\mathrm{C} 21-\mathrm{H} 21 \mathrm{C}$ & 109.5 \\
\hline $\mathrm{H} 21 \mathrm{~B}-\mathrm{C} 21-\mathrm{H} 21 \mathrm{C}$ & 109.5 \\
\hline C11-C22-H22A & 109.5 \\
\hline C11-C22-H22B & 109.5 \\
\hline $\mathrm{H} 22 \mathrm{~A}-\mathrm{C} 22-\mathrm{H} 22 \mathrm{~B}$ & 109.5 \\
\hline $\mathrm{C} 11-\mathrm{C} 22-\mathrm{H} 22 \mathrm{C}$ & 109.5 \\
\hline $\mathrm{H} 22 \mathrm{~A}-\mathrm{C} 22-\mathrm{H} 22 \mathrm{C}$ & 109.5 \\
\hline $\mathrm{H} 22 \mathrm{~B}-\mathrm{C} 22-\mathrm{H} 22 \mathrm{C}$ & 109.5 \\
\hline C28-C23-C24 & $118.89(8)$ \\
\hline C28-C23-N1 & $119.67(8)$ \\
\hline C24-C23-N1 & $121.45(8)$ \\
\hline $\mathrm{C} 25-\mathrm{C} 24-\mathrm{C} 23$ & $120.06(9)$ \\
\hline $\mathrm{C} 25-\mathrm{C} 24-\mathrm{H} 24$ & 120 \\
\hline $\mathrm{C} 23-\mathrm{C} 24-\mathrm{H} 24$ & 120 \\
\hline C26-C25-C24 & $120.75(9)$ \\
\hline C26-C25-H25 & 119.6 \\
\hline C24-C25-H25 & 119.6 \\
\hline $\mathrm{C} 25-\mathrm{C} 26-\mathrm{C} 27$ & $119.11(9)$ \\
\hline
\end{tabular}


$\mathrm{C} 25-\mathrm{C} 26-\mathrm{H} 26$

$\mathrm{C} 27-\mathrm{C} 26-\mathrm{H} 26$

$\mathrm{C} 28-\mathrm{C} 27-\mathrm{C} 26$

$\mathrm{C} 28-\mathrm{C} 27-\mathrm{H} 27$

$\mathrm{C} 26-\mathrm{C} 27-\mathrm{H} 27$

$\mathrm{C} 27-\mathrm{C} 28-\mathrm{C} 23$

$\mathrm{C} 27-\mathrm{C} 28-\mathrm{H} 28$

$\mathrm{C} 23-\mathrm{C} 28-\mathrm{H} 28$

Symmetry transformations used to generate equivalent atoms: \#1 -x,-y,-z+1
120.4

120.4

$120.66(9)$

119.7

119.7

120.53(9)

119.7

119.7 


\section{Synthesis and Characterization}

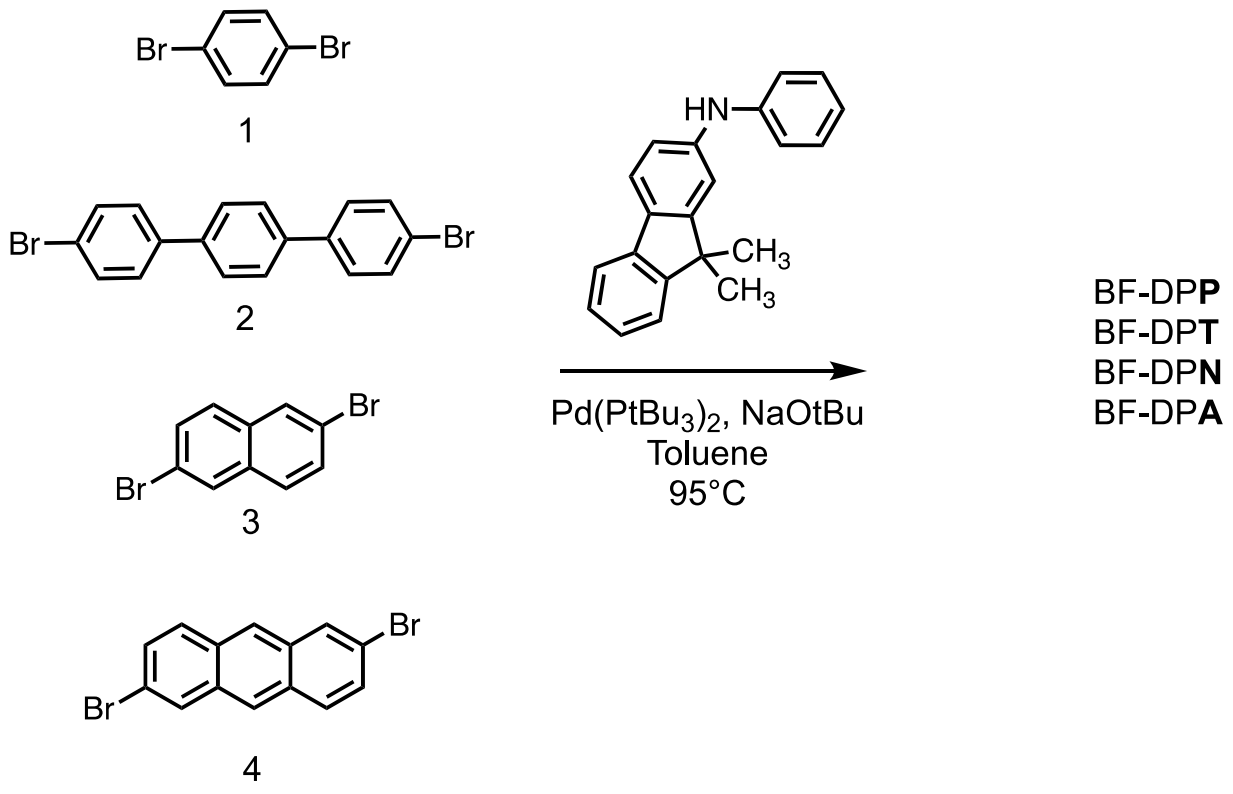

\section{General Procedure:}

The respective dibrominated linker $(1$ - 4) and 2-anilino-9,9-dimethylfluorene (1.00:2.01 molar equivalents) were added to a flame-dried round-bottom flask along with sodium tert-butoxide (3 equivalents) and the reagents were cycled three times between nitrogen gas and vacuum. The reaction was left under nitrogen, and anhydrous toluene was added to the flask (100 $\mathrm{mg}$ of dibrominated starting material $=10.0 \mathrm{ml}$ of toluene). The reaction was then sparged with nitrogen for thirty minutes. Bis(tri-tert-butylphosphine)palladium(0) was added to the flask ( 0.100 mol percent) and the reaction was again sparged for an additional fifteen minutes. The reaction was then sealed and placed in an oil bath at $95^{\circ} \mathrm{C}$ overnight. The reaction was cooled, filtered, and washed with copious amounts of diethyl ether. The solids were collected and subjected to sublimation for final purification. BF-DPP solids did not precipitate during the course of the reaction; a column with a solvent gradient of 50\% DCM 50\% hexanes was ran to obtain the desired product. All reactions ran on gram scales except BF-DPA, which was ran at $1.5 \mathrm{mmol} / 0.500 \mathrm{gr}$ scale.

BF-DPP Yellow solid, reaction yield $=87 \%$ (2.38 gr), sublimation yield $=34 \% .{ }^{\mathbf{1}} \mathbf{H N M R}(500$ MHz, 295K, DMSO) $\delta 7.74$ (d, 2H), 7.73 (d, 2H), 7.50 (d, J=7.4 Hz, 2H), 7.33-7.21 (br, 10H), 7.07 (d, J=8.0 Hz, 4H), 7.02-6.98 (br, 8H), 1.36 (s, 12H). ${ }^{13}$ CNMR (125 MHz, 295K, DMSO) $\delta$ $158.86,157.20,151.50,150.92$, 146.60, 142.39, 137.58, 133.61, 131.16, 130.69, 129.39, 127.21, 
126.76, 126.74, 126.64, 125.19, 123.61, 122.00, 50.50, 30.92. HRMS (MALDI +) calculated $\mathrm{m} / \mathrm{z}$ for $\left[\mathrm{C}_{48} \mathrm{H}_{40} \mathrm{~N}_{2}+\mathrm{H}\right]+$ is 644.32 , found 644.34 .

BF-DPT Yellow solid, reaction yield $=87 \%$ (2.19 gr), sublimation yield $=27 \% .{ }^{1}$ HNMR $(500$ $\mathrm{MHz}, 295 \mathrm{~K}, \mathrm{C}_{2} \mathrm{D}_{4} \mathrm{Cl}_{2}$ ) $\delta 7.67$ (br s, 4H), 7.65 (d, J=7.5 Hz, 2H), 7.62 (d, J=8.1 Hz, 2H), 7.57 (d, $\mathrm{J}=8.6 \mathrm{~Hz}, 4 \mathrm{H}), 7.40$ (d, J=7.4 Hz, 2H), 7.31-7.24 (br, 10H), 7.17 (dd, J=8.7, J=2.4 Hz, 8H), 7.077.04 (br, 4H), 1.42 (s, 12H). ${ }^{13} \mathbf{C N M R}\left(125 \mathrm{MHz}, 295 \mathrm{~K}, \mathrm{C}_{2} \mathrm{D}_{4} \mathrm{Cl}_{2}\right) \delta$ 129.69, 129.17, 127.80, $127.39,127.17,127.00,124.79,124.14,124.12$, 123.38, 122.90, 121.04, 119.81, 119.53, 47.18, 27.27. *the carbon spectra was not able to capture protons between 160 and $130 \mathrm{ppm}$ due to solubility. HRMS (MALDI +) calculated $\mathrm{m} / \mathrm{z}$ for $\left[\mathrm{C}_{60} \mathrm{H}_{48} \mathrm{~N}_{2}\right]+$ is 796.38 , found 796.39 .

BF-DPN Yellow solid, reaction yield $=100 \%$ (2.45 gr), sublimation yield $=70 \% .{ }^{1} \mathbf{H N M R}(500$ $\left.\mathrm{MHz}, 295 \mathrm{~K}, \mathrm{C}_{2} \mathrm{D}_{4} \mathrm{Cl}_{2}\right) \delta 7.85(\mathrm{~d}, \mathrm{~J}=7.4 \mathrm{~Hz}, 2 \mathrm{H}), 7.62$ (d, J=8.1 Hz, 2H), 7.51 (d, J=8.7 Hz, 2H), 7.41 (d, J=6.7 Hz, 4H), 7.31-7.23 (br, 12H), 7.15 (d, J=7.9 Hz, 4H), 7.05-7.02 (br, 4H), 1.36 (s, 12H). ${ }^{13}$ CNMR (125 MHz, 295K, $\left.\mathrm{C}_{2} \mathrm{D}_{4} \mathrm{Cl}_{2}\right) \delta$ 155.41, 153.85, 148.21, 147.51, 144.80, 140.07, $139.12,134.40,131.20,129.63,128.10,127.29,126.81,125.12,124.29,123.68,123.01,122.80$, 120.91, 120.39, 119.64, 119.02, 46.98, 27.13. HRMS (MALDI +) calculated m/z for $\left[\mathrm{C}_{52} \mathrm{H}_{42} \mathrm{~N}_{2}+\mathrm{H}\right]+$ is 694.33 , found 694.27 .

BF-DPA Yellow solid, reaction yield $=70 \%(0.765$ gr $)$, sublimation yield $=69 \% .{ }^{1} \mathbf{H N M R}(500$ $\mathrm{MHz}, 295 \mathrm{~K}, \mathrm{C}_{2} \mathrm{D}_{4} \mathrm{Cl}_{2}$ ) $\delta 8.04$ (s br, 2H), 7.78 (d, 2H), 7.65 (d, 2H), 7.63 (d, J=8.0 Hz, 2H), 7.51 (d, 2H), 7.41 (d, J=6.9 Hz, 2H), 7.32-7.26 (s br, 12H), 7.20 (d, J=7.9 Hz, 4H), 7.10-7.07 (br, 4H), $1.40(\mathrm{~s}, 12 \mathrm{H}) . *$ carbon spectra could not be obtained due to solubility. HRMS (MALDI +) calculated $\mathrm{m} / \mathrm{z}$ for $\left[\mathrm{C}_{56} \mathrm{H}_{44} \mathrm{~N}_{2}+\mathrm{H}\right]+$ is 744.35 , found 744.13 . 
MALDI Spectra for new donor materials.
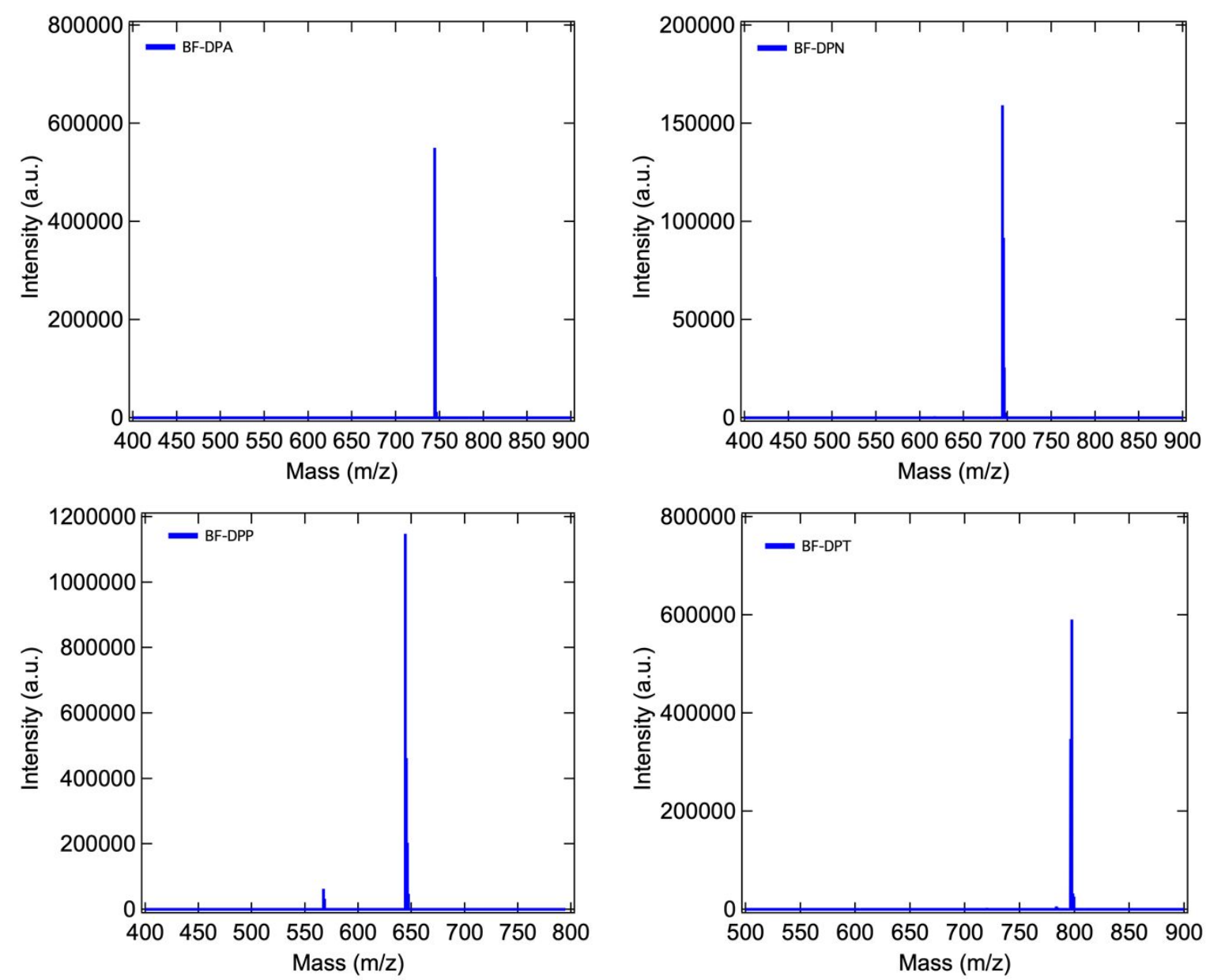


\section{NMR Spectra}

\section{BF-DPP}

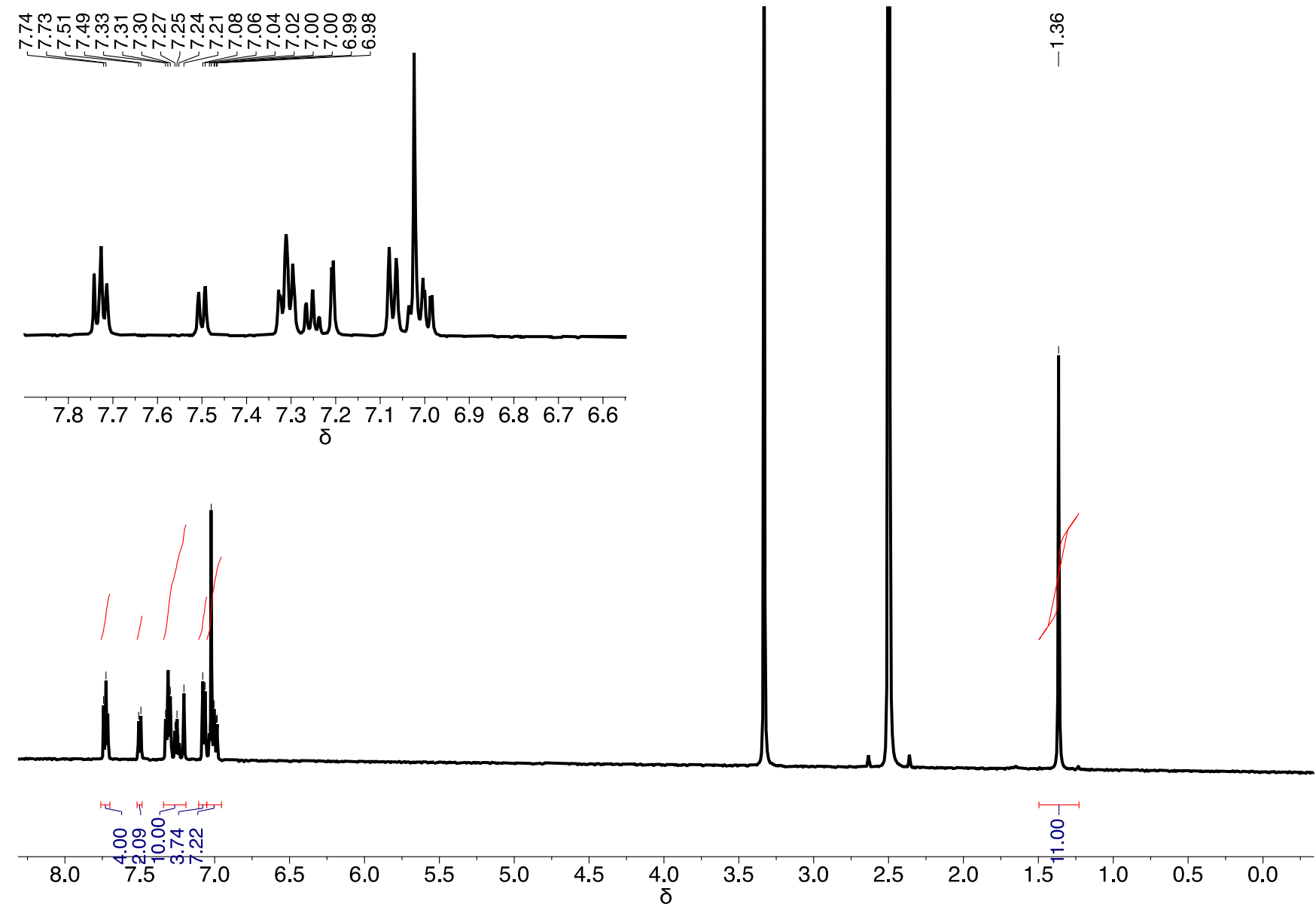




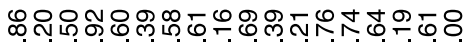

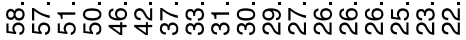

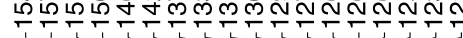

๙ั.

iे

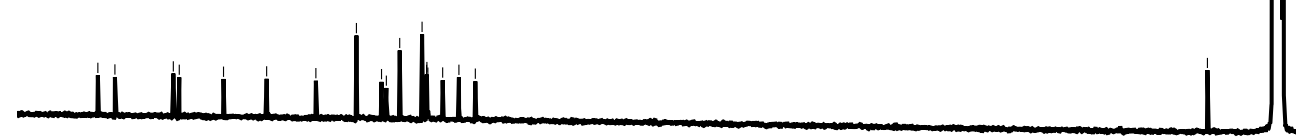

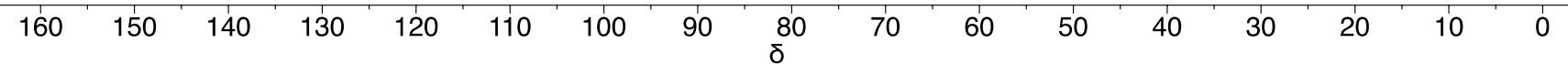




\section{BF-DPT}

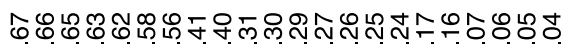

NNNNNNNNNNNNNNNNNN
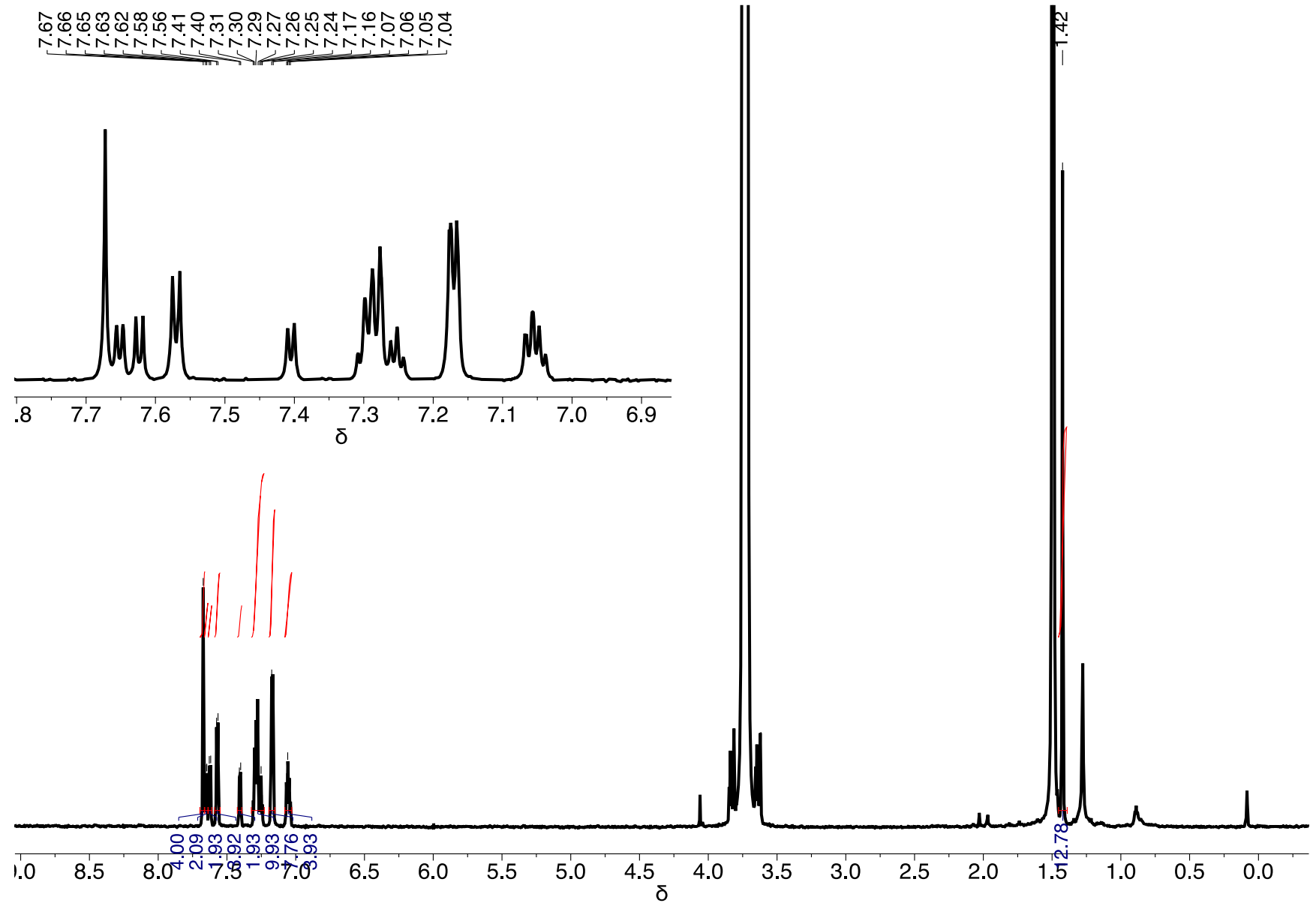


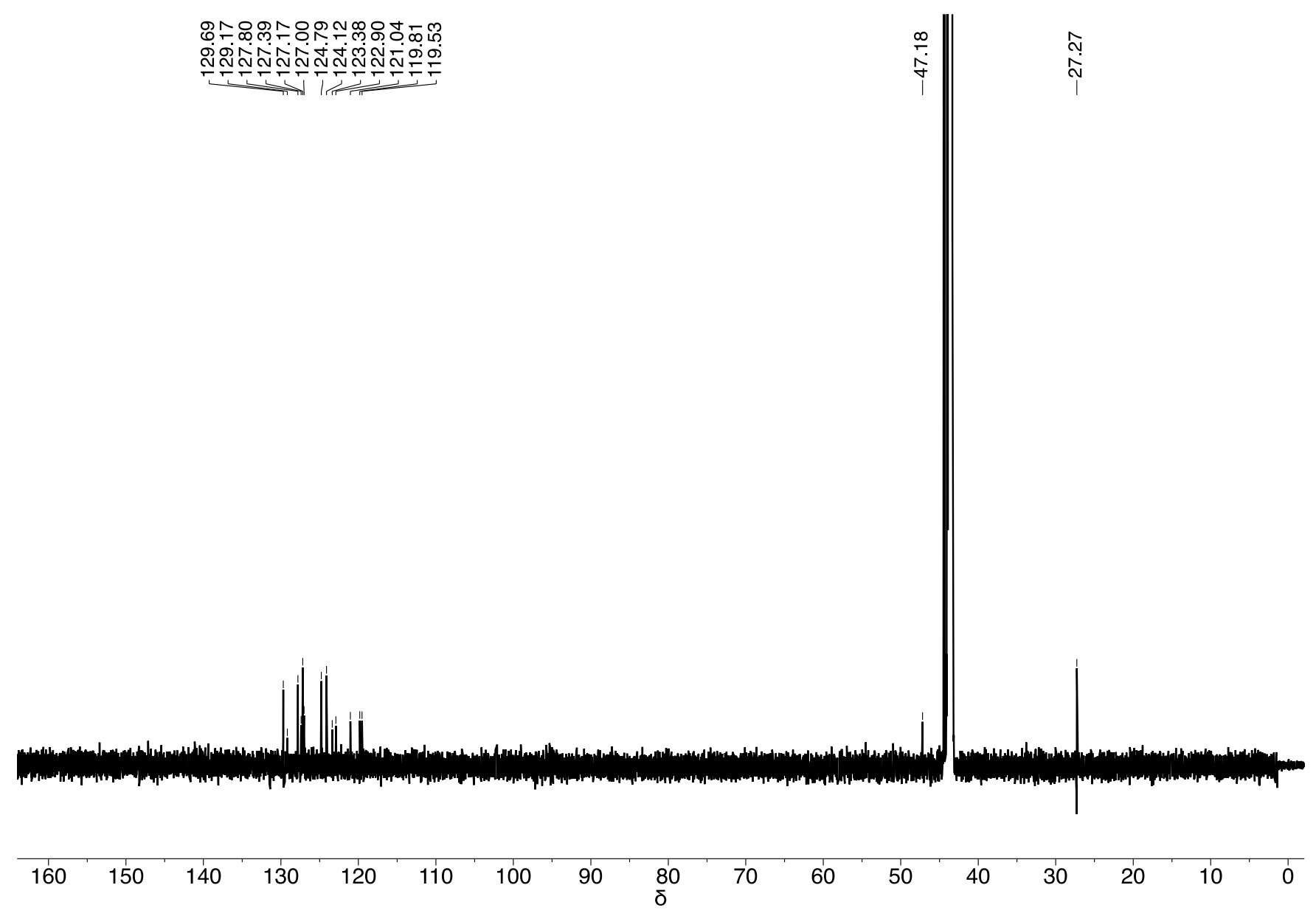




\section{BF-DPN}

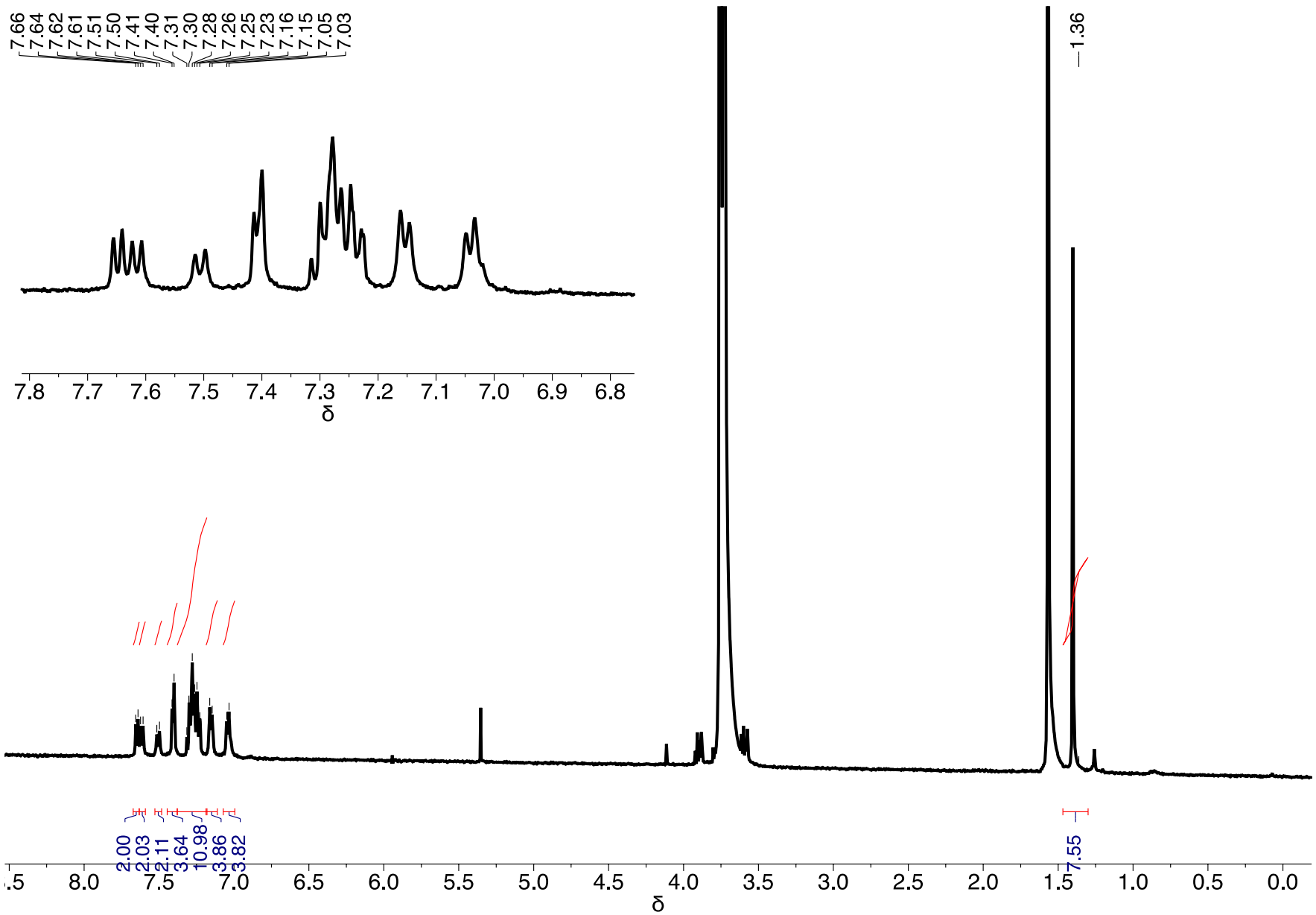




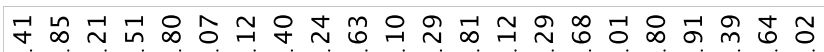

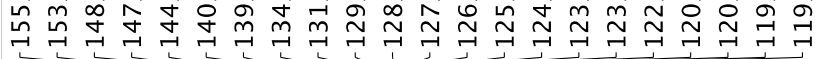

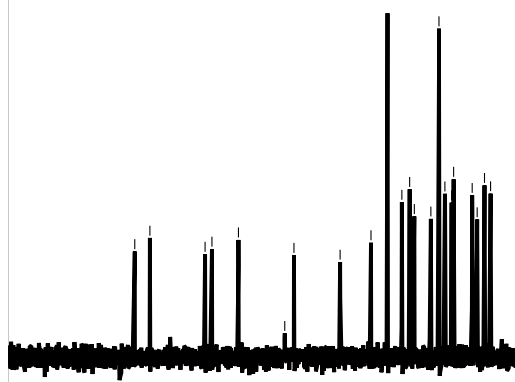

16

150

$140 \quad 130$

120

$10 \quad 100$

90

80

$\delta$

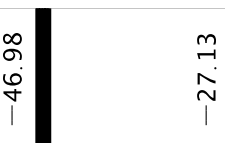

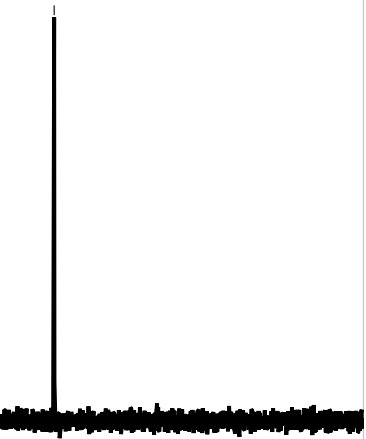




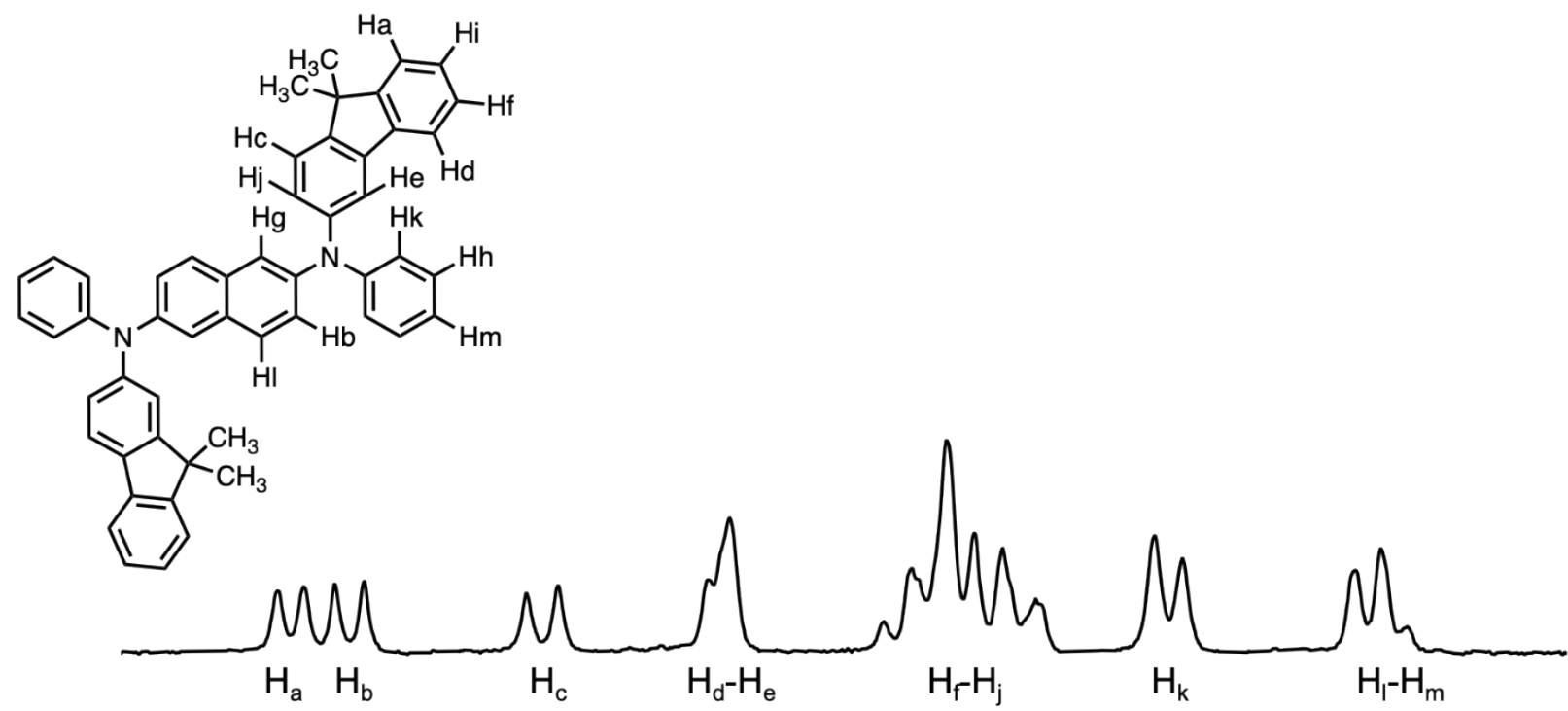

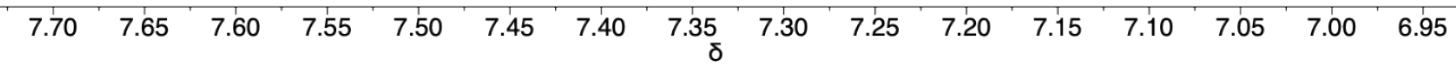

Proton Assignment for BF-DPN. There are 30 aromatic protons with the following assignment: $\mathrm{Ha}=2 \mathrm{H}, \mathrm{Hb}=2 \mathrm{H}, \mathrm{Hc}=2 \mathrm{H}), \mathrm{Hd}=2 \mathrm{H}$ and $\mathrm{He}=2 \mathrm{H}, \mathrm{Hf}-\mathrm{Hj}=12 \mathrm{H}, \mathrm{Hk}=4 \mathrm{H}), \mathrm{Hl}=2 \mathrm{H}$ and $\mathrm{Hm}$ $=2 \mathrm{H}$.

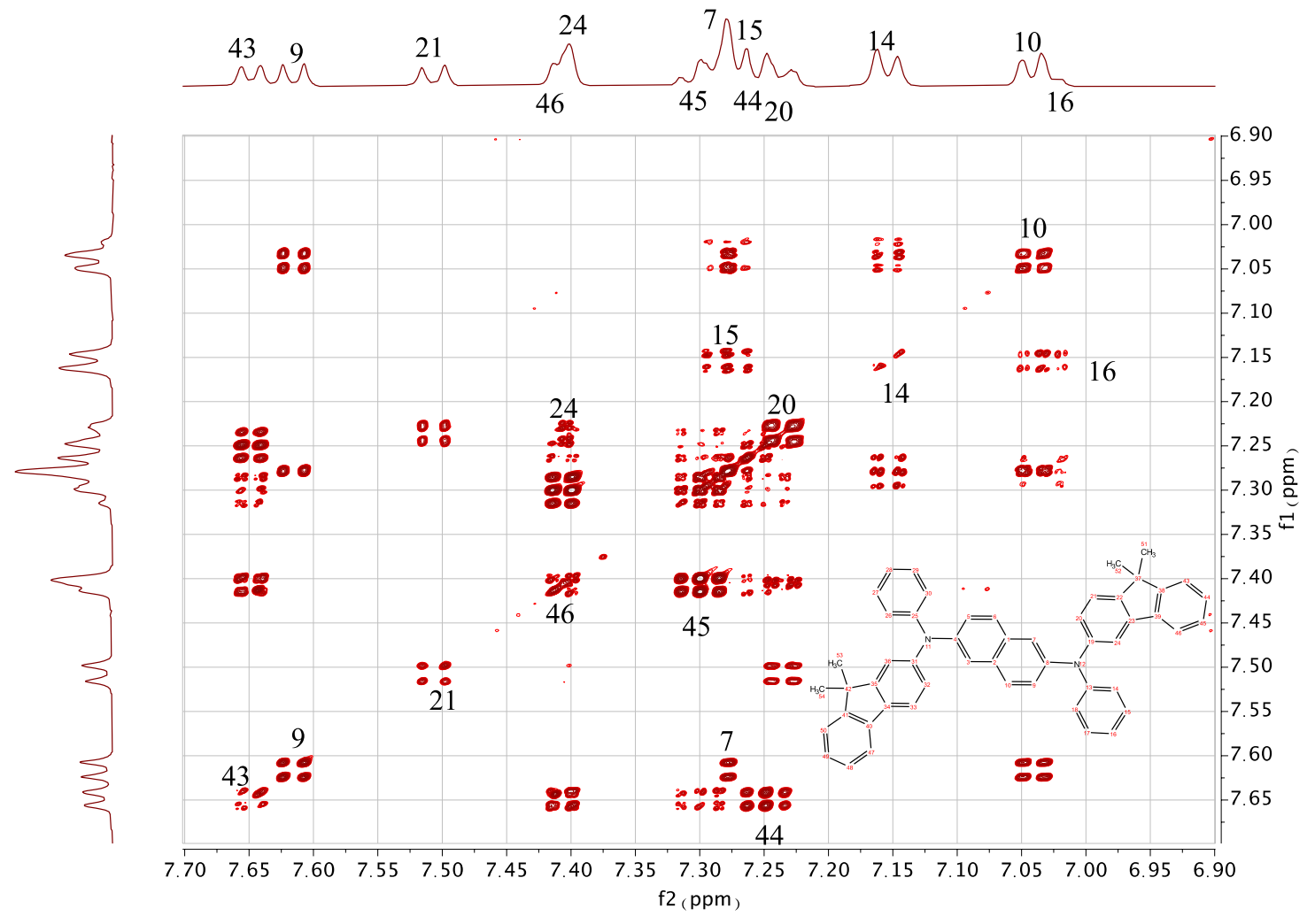

COSY NMR for BF-DPN 


\section{BF-DPA}

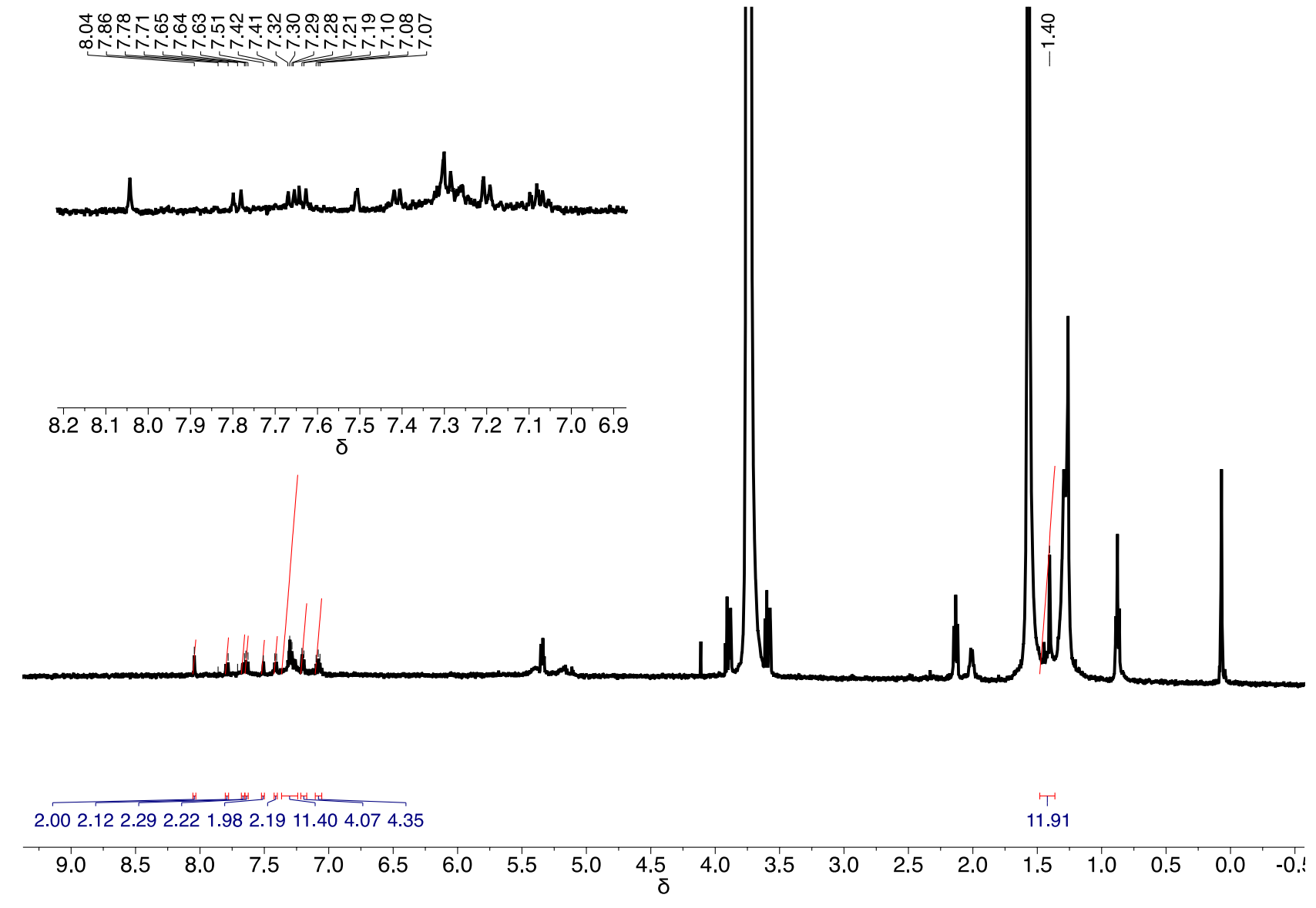




\section{Density Functional Theory}

Geometry optimizations and time-dependent density functional theory performed by Schrodinger software version 2021-2, and the associated reference is as follows: Bochevarov, A.D.; Harder, E.; Hughes, T.F.; Greenwood, J.R.; Braden, D.A.; Philipp, D.M.; Rinaldo, D.; Halls, M.D.; Zhang, J.; Friesner, R.A., "Jaguar: A high-performance quantum chemistry software program with strengths in life and materials sciences," Int. J. Quantum Chem., 2013, 113(18), 2110-2142. Both geometry optimizations and time-dependent calculations used B3LYP-D3/6-31 ${ }^{++} \mathrm{G}^{* *}$. The following pages contain the xyz coordinates of the lowest energy geometry and the detailed timedependent data for the first fifteen roots.

\begin{tabular}{lrrr}
\multicolumn{4}{l}{ Lowest Energy Geometries } \\
BF-DPP -1962.9671 hartrees \\
B & -0.66464 & -1.68849 & 0.08843 \\
C & 0.06282 & -2.87337 & 0.29235 \\
C & 1.42642 & -2.83540 & 0.56347 \\
C & 2.09667 & -1.60823 & 0.67978 \\
C & 1.36143 & -0.42141 & 0.51818 \\
C & 0.00549 & -0.45926 & 0.20805 \\
C & -2.96447 & -0.79359 & 0.23653 \\
C & -2.47485 & -2.72861 & -1.18996 \\
C & 4.10448 & -2.48028 & 1.81149 \\
C & 4.29646 & -0.66108 & 0.17406 \\
C & 5.34194 & -3.06577 & 1.49487 \\
C & 5.93841 & -3.96707 & 2.37786 \\
C & 5.30658 & -4.31157 & 3.57609 \\
C & 4.06978 & -3.73790 & 3.88842 \\
C & 3.47442 & -2.82296 & 3.02062 \\
C & -3.67192 & -3.42873 & -0.96627 \\
C & -4.10730 & -4.38522 & -1.88278 \\
C & -3.35125 & -4.67037 & -3.02427 \\
C & -2.15587 & -3.97936 & -3.24431 \\
C & -1.72130 & -3.00896 & -2.34112 \\
C & -3.93914 & -0.25185 & -0.62378 \\
C & -4.87955 & 0.66842 & -0.15106 \\
C & -4.83862 & 1.06840 & 1.18027 \\
C & -3.85305 & 0.54068 & 2.03751 \\
C & -2.92185 & -0.39166 & 1.58187 \\
C & 4.08032 & -0.50638 & -1.20702 \\
C & 4.86781 & 0.38552 & -1.91930 \\
C & 5.88199 & 1.12675 & -1.28191 \\
C & 6.10725 & 0.96772 & 0.08912 \\
C & 5.30944 & 0.08071 & 0.81109 \\
C & -4.03707 & 1.12777 & 3.37198 \\
C & -5.13302 & 2.01287 & 3.32314 \\
C & -5.74053 & 2.05052 & 1.92211 \\
C & 6.53390 & 1.98015 & -2.28099
\end{tabular}




$\begin{array}{crrr}\mathrm{C} & 5.92250 & 1.75251 & -3.53143 \\ \mathrm{C} & 4.80998 & 0.71284 & -3.40936 \\ \mathrm{C} & 6.35082 & 2.44181 & -4.66073 \\ \mathrm{C} & 7.39667 & 3.36806 & -4.53769 \\ \mathrm{C} & 8.00342 & 3.59351 & -3.29537 \\ \mathrm{C} & 7.57773 & 2.90116 & -2.15732 \\ \mathrm{C} & -5.52523 & 2.70965 & 4.46144 \\ \mathrm{C} & -4.81529 & 2.51739 & 5.65468 \\ \mathrm{C} & -3.72649 & 1.63719 & 5.70235 \\ \mathrm{C} & -3.32762 & 0.93532 & 4.56035 \\ \mathrm{C} & -5.65614 & 3.46594 & 1.31021 \\ \mathrm{C} & -7.20762 & 1.56636 & 1.93812 \\ \mathrm{C} & 5.10812 & -0.53491 & -4.26908 \\ \mathrm{C} & 3.43867 & 1.30878 & -3.79317 \\ \mathrm{H} & -0.44129 & -3.83007 & 0.20435 \\ \mathrm{H} & 1.97583 & -3.76142 & 0.69659 \\ \mathrm{H} & 1.86794 & 0.53426 & 0.60538 \\ \mathrm{H} & -0.54178 & 0.46636 & 0.06385 \\ \mathrm{H} & 5.83117 & -2.80851 & 0.56151 \\ \mathrm{H} & 6.89617 & -4.40997 & 2.12032 \\ \mathrm{H} & 3.56956 & -3.99241 & 4.81853 \\ \mathrm{H} & 2.51986 & -2.37052 & 3.26901 \\ \mathrm{H} & -4.25376 & -3.21246 & -0.07606 \\ \mathrm{H} & -5.03470 & -4.91944 & -1.69678 \\ \mathrm{H} & -3.68890 & -5.42144 & -3.73183 \\ \mathrm{H} & -1.56261 & -4.18699 & -4.13030 \\ \mathrm{H} & -0.79663 & -2.46877 & -2.51629 \\ \mathrm{H} & -3.95758 & -0.56241 & -1.66287 \\ \mathrm{H} & -5.62484 & 1.07112 & -0.83212 \\ \mathrm{H} & -2.17146 & -0.80863 & 2.24596 \\ \mathrm{H} & 3.29853 & -1.08034 & -1.69476 \\ \mathrm{H} & 6.88108 & 1.53639 & 0.59701 \\ \mathrm{H} & 5.46093 & -0.04851 & 1.87776 \\ \mathrm{H} & 5.88285 & 2.27070 & -5.62688 \\ \mathrm{H} & 7.73921 & 3.91465 & -5.41149 \\ \mathrm{H} & 8.81192 & 4.31456 & -3.21551 \\ \mathrm{H} & 8.04967 & 3.07938 & -1.19547 \\ \mathrm{H} & -6.36903 & 3.39427 & 4.43130 \\ \mathrm{H} & -5.11202 & 3.05474 & 6.55086 \\ \mathrm{H} & -3.18705 & 1.50086 & 6.63522 \\ \mathrm{H} & -2.48147 & 0.25482 & 4.59828 \\ \mathrm{H} & -4.62207 & 3.82320 & 1.29616 \\ \mathrm{H} & -6.03358 & 3.46179 & 0.28179 \\ \mathrm{H} & -6.25769 & 4.17309 & 1.89126 \\ \mathrm{H} & -7.28256 & 0.56473 & 2.37224 \\ \mathrm{H} & -7.83091 & 2.24590 & 2.52964\end{array}$




$\begin{array}{rrrr}\mathrm{H} & -7.61083 & 1.53194 & 0.92042 \\ \mathrm{H} & 6.07676 & -0.96874 & -4.00257 \\ \mathrm{H} & 4.33588 & -1.29741 & -4.11976 \\ \mathrm{H} & 5.12891 & -0.27230 & -5.33225 \\ \mathrm{H} & 3.21721 & 2.19529 & -3.19117 \\ \mathrm{H} & 3.42723 & 1.59833 & -4.84950 \\ \mathrm{H} & 2.64234 & 0.57478 & -3.63022 \\ \mathrm{H} & 5.76960 & -5.01769 & 4.25943 \\ \mathrm{~N} & -2.03866 & -1.75143 & -0.25583 \\ \mathrm{~N} & 3.49275 & -1.55323 & 0.93137\end{array}$

BF-DPB - 2194.0487 hartrees

\begin{tabular}{|c|c|c|c|}
\hline & -2.68480 & -0.94536 & \\
\hline & -1.94273 & -2.10997 & 95679 \\
\hline & -0.55295 & -2.06970 & \\
\hline & & -0.87019 & 76386 \\
\hline & -0.60590 & 0.2 & \\
\hline & -1.99733 & 277 & \\
\hline & 1.62911 & -0.8 & \\
\hline & 2.32977 & $-0.0^{\prime}$ & \\
\hline & 3.72 & & \\
\hline & 4.46 & -0.8 & \\
\hline & 3.775 & -1.6 & \\
\hline & 2.38440 & -1.6 & \\
\hline & -4.899 & 0.0 & \\
\hline 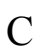 & -4.71 & -2.1 & \\
\hline & 6.669 & -0.9 & 09 \\
\hline$y$ & $6.50^{\circ}$ & -0.8 & \\
\hline & 7.846 & -1.7 & 13 \\
\hline & 8.628 & -1.7 & \\
\hline & 8.247 & -1.0 & 76 \\
\hline & 7.071 & -0.2 & \\
\hline & 6.290 & -0.2 & \\
\hline & -5.86751 & -2.6 & \\
\hline & -6.47808 & -3.7 & -0.07249 \\
\hline & -5.94794 & -4.3 & -1.24948 \\
\hline & -4.80073 & -3.7 & -1.80656 \\
\hline & -4.19307 & -2.6 & -1.20256 \\
\hline C & -6.01082 & 0.5 & 0.41659 \\
\hline & -6.83457 & 1.5 & \\
\hline $\mathrm{C}$ & -6.53803 & 2.06229 & 2.18056 \\
\hline$C$ & -5.41466 & 1.60755 & 2.89828 \\
\hline $\mathrm{C}$ & -4.59690 & 0.59664 & 2.39567 \\
\hline C & 6.04270 & -1.73118 & -1.72912 \\
\hline$C$ & & -1.70231 & \\
\hline & 7.77060 & -0.85283 & -3.20000 \\
\hline
\end{tabular}




$\begin{array}{rrrr}\mathrm{C} & 8.24048 & -0.02001 & -2.18066 \\ \mathrm{C} & 7.60223 & -0.03472 & -0.94130 \\ \mathrm{C} & -5.34722 & 2.33985 & 4.16969 \\ \mathrm{C} & -6.43261 & 3.23737 & 4.22522 \\ \mathrm{C} & -7.28058 & 3.14519 & 2.95744 \\ \mathrm{C} & 8.22452 & -1.04476 & -4.58158 \\ \mathrm{C} & 7.40352 & -2.01628 & -5.19004 \\ \mathrm{C} & 6.34738 & -2.52492 & -4.21052 \\ \mathrm{C} & 7.61789 & -2.39109 & -6.51167 \\ \mathrm{C} & 8.66260 & -1.79106 & -7.22874 \\ \mathrm{C} & 9.47802 & -0.82529 & -6.62497 \\ \mathrm{C} & 9.26510 & -0.44428 & -5.29608 \\ \mathrm{C} & -6.61418 & 4.05090 & 5.33884 \\ \mathrm{C} & -5.70348 & 3.96312 & 6.40105 \\ \mathrm{C} & -4.62059 & 3.07620 & 6.34111 \\ \mathrm{C} & -4.43407 & 2.25583 & 5.22400 \\ \mathrm{C} & -7.28483 & 4.48481 & 2.18885 \\ \mathrm{C} & -8.72778 & 2.71399 & 3.27956 \\ \mathrm{C} & 6.51950 & -4.03548 & -3.93881 \\ \mathrm{C} & 4.91958 & -2.23328 & -4.71721 \\ \mathrm{H} & -2.46201 & -3.04948 & 1.11399 \\ \mathrm{H} & -0.00205 & -2.99051 & 1.14657 \\ \mathrm{H} & -0.09608 & 1.24302 & 0.39478 \\ \mathrm{H} & -2.55865 & 1.17291 & 0.31358 \\ \mathrm{H} & 1.77729 & 0.52322 & -0.91440 \\ \mathrm{H} & 4.24015 & 0.52488 & -0.98285 \\ \mathrm{H} & 4.33642 & -2.26619 & 2.28504 \\ \mathrm{H} & 1.87582 & -2.22919 & 2.38896 \\ \mathrm{H} & 8.14383 & -2.26624 & 0.90296 \\ \mathrm{H} & 9.53602 & -2.32806 & 2.94636 \\ \mathrm{H} & 8.85610 & -1.04442 & 4.97099 \\ \mathrm{H} & 6.76502 & 0.30955 & 4.91395 \\ \mathrm{H} & 5.38617 & 0.39019 & 2.86104 \\ \mathrm{H} & -6.27491 & -2.28692 & 1.46669 \\ \mathrm{H} & --7.36480 & -4.22564 & 0.37543 \\ \mathrm{H} & -6.42139 & -5.17655 & -1.72559 \\ \mathrm{H} & -4.38167 & -4.15197 & -2.72462 \\ \mathrm{H} & -3.30804 & -2.20107 & -1.64178 \\ \mathrm{H} & -6.22712 & 0.07252 & -0.55275 \\ \mathrm{H} & -7.69232 & 1.85336 & 0.36574 \\ \mathrm{H} & -3.74242 & 0.23115 & 2.95558 \\ \mathrm{H} & 5.19847 & -2.38732 & -1.53993 \\ \mathrm{H} & 9.08311 & 0.64493 & -2.34823 \\ \mathrm{H} & 7.94607 & 0.61191 & -0.14078 \\ \mathrm{H} & 6.98824 & -3.13891 & -6.98688 \\ \mathrm{H} & 8.83992 & -2.07400 & -8.26233\end{array}$




$\begin{array}{cccc}\mathrm{H} & 10.28286 & -0.36874 & -7.19402 \\ \mathrm{H} & 9.90019 & 0.30314 & -4.82948 \\ \mathrm{H} & -7.45021 & 4.74357 & 5.39149 \\ \mathrm{H} & -5.83550 & 4.59155 & 7.27715 \\ \mathrm{H} & -3.92302 & 3.02293 & 7.17198 \\ \mathrm{H} & -3.59598 & 1.56579 & 5.17991 \\ \mathrm{H} & -6.26439 & 4.80346 & 1.95562 \\ \mathrm{H} & -7.83836 & 4.38549 & 1.24871 \\ \mathrm{H} & -7.76303 & 5.26904 & 2.78535 \\ \mathrm{H} & -8.73789 & 1.76644 & 3.82594 \\ \mathrm{H} & -9.22848 & 3.47100 & 3.89297 \\ \mathrm{H} & -9.30494 & 2.58594 & 2.35727 \\ \mathrm{H} & 7.52850 & -4.25304 & -3.57553 \\ \mathrm{H} & 5.80037 & -4.37510 & -3.18548 \\ \mathrm{H} & 6.35234 & -4.61088 & -4.85538 \\ \mathrm{H} & 4.78665 & -1.16540 & -4.91556 \\ \mathrm{H} & 4.72133 & -2.78217 & -5.64410 \\ \mathrm{H} & 4.17771 & -2.54026 & -3.97243 \\ \mathrm{~N} & -4.09709 & -1.00310 & 0.61008 \\ \mathrm{~N} & 5.87738 & -0.88961 & 0.56871\end{array}$

BF-DPT - 2425.1292 hartrees

\begin{tabular}{|c|c|c|c|}
\hline & -4.62868 & & \\
\hline & -3.78361 & & \\
\hline & -2.40937 & -0.21248 & \\
\hline & -1.81924 & & 6284 \\
\hline & -2.67475 & -1.8 & 0007 \\
\hline & 467 & -1.8 & 262 \\
\hline & & -0.9 & \\
\hline & 4.5 & -0.2 & 550 \\
\hline & & -0.1 & \\
\hline & 6.77 & -0.6 & \\
\hline & & -1.4 & \\
\hline & 4.8 & -1.5 & \\
\hline & -6.6 & 0.2 & \\
\hline & -6.77 & -2.2 & 197 \\
\hline & 8.88 & -0.2 & 272 \\
\hline & 8.89 & -0.5 & \\
\hline & 10.11471 & -0 & 441 \\
\hline & 10.80370 & -0.5 & 2 . \\
\hline & & 0.3 & 9055 \\
\hline & 9.04255 & 0.98529 & 3.42569 \\
\hline & 8.35362 & 0.70 & \\
\hline & -8.03303 & -2.26903 & \\
\hline & -8.75807 & -3.46078 & \\
\hline & -8.23446 & -4.61616 & 1.54972 \\
\hline
\end{tabular}




$\begin{array}{cccc}\mathrm{C} & -6.97587 & -4.56262 & 2.15529 \\ \mathrm{C} & -6.25066 & -3.37144 & 2.18182 \\ \mathrm{C} & -6.35558 & 1.36726 & 1.05545 \\ \mathrm{C} & -6.97205 & 2.60042 & 1.28592 \\ \mathrm{C} & -7.93758 & 2.71255 & 2.28219 \\ \mathrm{C} & -8.27715 & 1.58160 & 3.05045 \\ \mathrm{C} & -7.65479 & 0.35076 & 2.84045 \\ \mathrm{C} & 8.58162 & -1.55975 & -2.06200 \\ \mathrm{C} & 9.28913 & -1.61874 & -3.25532 \\ \mathrm{C} & 10.31921 & -0.69683 & -3.53058 \\ \mathrm{C} & 10.62846 & 0.29969 & -2.60001 \\ \mathrm{C} & 9.90994 & 0.37139 & -1.40641 \\ \mathrm{C} & -9.30714 & 1.97267 & 4.02411 \\ \mathrm{C} & -9.59300 & 3.34189 & 3.84688 \\ \mathrm{C} & -8.74414 & 3.93497 & 2.71937 \\ \mathrm{C} & 10.89425 & -1.01307 & -4.84405 \\ \mathrm{C} & 10.21608 & -2.13214 & -5.37066 \\ \mathrm{C} & 9.12939 & -2.61368 & -4.40746 \\ \mathrm{C} & 10.57060 & -2.64706 & -6.61392 \\ \mathrm{C} & 11.60791 & -2.03908 & -7.33459 \\ \mathrm{C} & 12.27978 & -0.92732 & -6.81230 \\ \mathrm{C} & 11.92875 & -0.40626 & -5.56324 \\ \mathrm{C} & -10.54525 & 3.96706 & 4.64768 \\ \mathrm{C} & -11.21237 & 3.21798 & 5.62696 \\ \mathrm{C} & -10.92541 & 1.85856 & 5.80141 \\ \mathrm{C} & -9.97097 & 1.22507 & 5.00076 \\ \mathrm{C} & -7.82028 & 5.05887 & 3.24321 \\ \mathrm{C} & -9.62921 & 4.47107 & 1.57082 \\ \mathrm{C} & 9.38608 & -4.06503 & -3.94503 \\ \mathrm{C} & 7.72699 & -2.50574 & -5.04748 \\ \mathrm{C} & 2.48477 & -1.01378 & 0.45553 \\ \mathrm{C} & 1.89196 & -0.91975 & 1.72660 \\ \mathrm{C} & 0.51085 & -0.94268 & 1.88414 \\ \mathrm{C} & -0.34871 & -1.05280 & 0.77747 \\ \mathrm{C} & 0.24282 & -1.15894 & -0.49426 \\ \mathrm{C} & 1.62764 & -1.14141 & -0.65180 \\ \mathrm{H} & -4.21019 & 0.45009 & 2.89696 \\ \mathrm{H} & -1.78324 & 0.44617 & 2.52482 \\ \mathrm{H} & -2.25556 & -2.52575 & -0.54733 \\ \mathrm{H} & -4.69133 & -2.48634 & -0.20785 \\ \mathrm{H} & 3.93968 & 0.21631 & -1.50665 \\ \mathrm{H} & 6.38249 & 0.44592 & -1.65647 \\ \mathrm{H} & 6.81789 & -1.86717 & 1.94313 \\ \mathrm{H} & 4.37689 & -2.10682 & 2.08627 \\ \mathrm{H} & 10.52653 & -1.52384 & 0.87042 \\ \mathrm{H} & 11.75513 & -1.00986 & 2.95665\end{array}$




$\begin{array}{cccc}\mathrm{H} & 10.80806 & 0.59607 & 4.60812 \\ \mathrm{H} & 8.62114 & 1.69105 & 4.13572 \\ \mathrm{H} & 7.40460 & 1.19378 & 2.03919 \\ \mathrm{H} & -8.43917 & -1.37897 & 0.49903 \\ \mathrm{H} & -9.73181 & -3.48857 & 0.48095 \\ \mathrm{H} & -8.79830 & -5.54390 & 1.53520 \\ \mathrm{H} & -6.55825 & -5.45000 & 2.62267 \\ \mathrm{H} & -5.27907 & -3.33477 & 2.66355 \\ \mathrm{H} & -5.61224 & 1.27102 & 0.27109 \\ \mathrm{H} & -6.69722 & 3.45398 & 0.67212 \\ \mathrm{H} & -7.90671 & -0.51579 & 3.44281 \\ \mathrm{H} & 7.79809 & -2.27710 & -1.83619 \\ \mathrm{H} & 11.41207 & 1.02583 & -2.79812 \\ \mathrm{H} & 10.13690 & 1.14695 & -0.68253 \\ \mathrm{H} & 10.05600 & -3.51001 & -7.02953 \\ \mathrm{H} & 11.89284 & -2.43342 & -8.30579 \\ \mathrm{H} & 13.08071 & -0.46604 & -7.38309 \\ \mathrm{H} & 12.45431 & 0.45613 & -5.16359 \\ \mathrm{H} & -10.77627 & 5.02202 & 4.52228 \\ \mathrm{H} & -11.95640 & 3.69657 & 6.25735 \\ \mathrm{H} & -11.45146 & 1.29133 & 6.56398 \\ \mathrm{H} & -9.75534 & 0.16940 & 5.13750 \\ \mathrm{H} & -7.18745 & 4.69819 & 4.05973 \\ \mathrm{H} & -7.16962 & 5.42941 & 2.44345 \\ \mathrm{H} & -8.41077 & 5.90293 & 3.61541 \\ \mathrm{H} & -10.29043 & 3.68837 & 1.18662 \\ \mathrm{H} & -10.25022 & 5.30456 & 1.91707 \\ \mathrm{H} & -9.00988 & 4.83359 & 0.74305 \\ \mathrm{H} & 10.37299 & -4.16219 & -3.48279 \\ \mathrm{H} & 8.63236 & -4.37728 & -3.21438 \\ \mathrm{H} & 9.33744 & -4.75514 & -4.79430 \\ \mathrm{H} & 7.52663 & -1.48455 & -5.38574 \\ \mathrm{H} & 7.64543 & -3.17359 & -5.91155 \\ \mathrm{H} & 6.95081 & -2.78799 & -4.32830 \\ \mathrm{H} & 2.52080 & -0.79932 & 2.60308 \\ \mathrm{H} & 0.09216 & -0.88289 & 2.88401 \\ \mathrm{H} & -0.38854 & -1.23375 & -1.37447 \\ \mathrm{H} & 2.04863 & -1.23128 & -1.64903 \\ \mathrm{~N} & -6.02928 & -1.00092 & 1.59462 \\ \mathrm{~N} & 8.18350 & -0.49411 & 0.10895\end{array}$

BF-DPN - 2116.6267 hartrees
$\begin{array}{llll}\text { C } & -0.48425 & 0.03997 & -0.00927\end{array}$
$\begin{array}{llll}\text { C } & 0.28303 & 1.21365 & -0.25935\end{array}$
$\begin{array}{llll}\text { C } & -0.36320 & 2.38780 & -0.60680\end{array}$
$\begin{array}{llll}\text { C } & -1.77227 & 2.43349 & -0.73598\end{array}$ 


\begin{tabular}{|c|c|c|c|}
\hline & & & \\
\hline & & & \\
\hline & -2.45909 & & \\
\hline & & & \\
\hline & -4.60054 & & \\
\hline & & & \\
\hline & & & \\
\hline & 2.29342 & & \\
\hline & -6.69747 & & \\
\hline & -6.7 & & 368 \\
\hline & & & \\
\hline & & & \\
\hline & & & \\
\hline & & & \\
\hline & & & \\
\hline & -7.7 & & \\
\hline & & & \\
\hline & -8.0 & & \\
\hline & -6.9 & & \\
\hline & & & \\
\hline & -6.2 & & -2.7 \\
\hline & & & \\
\hline & -8.2 & & $-2 . \varepsilon$ \\
\hline & -8.7 & & -1.6 \\
\hline & -7.9 & & \\
\hline & 1.7 & & \\
\hline & 2.2 & & \\
\hline & 3.4 & -0. & \\
\hline & & & \\
\hline & & & \\
\hline & & & \\
\hline & & & \\
\hline & 1.8 & -0.2 & \\
\hline & & & \\
\hline & -10 & & \\
\hline & -9.1 & -1.6 & \\
\hline & & -1.6 & \\
\hline & 4.13217 & -2.4 & \\
\hline & & -2.8 & \\
\hline & 4.95011 & -2.36 & 4.64240 \\
\hline & -9.1 & -2.54 & -4.2 \\
\hline & -10.26607 & & \\
\hline & -11.3 & -3.34742 & -3.51305 \\
\hline & & -2.40699 & -2.47326 \\
\hline & & 1.09435 & 5.36478 \\
\hline & 0.44266 & -0.95698 & 4.77972 \\
\hline
\end{tabular}




\begin{tabular}{|c|c|c|c|}
\hline & & & \\
\hline $\mathrm{C}$ & & & \\
\hline & & & \\
\hline & & & \\
\hline & -2.42870 & -0.83774 & \\
\hline & -1.8 & & \\
\hline & -4.3 & & \\
\hline & -4.5 & & \\
\hline & & & \\
\hline & & & \\
\hline & & & \\
\hline & 2.6 & & \\
\hline & & & \\
\hline & -8.0 & & \\
\hline & -9.2 & & -1.2 \\
\hline & -8.5 & & \\
\hline & & & \\
\hline & & & \\
\hline & -5.2 & & -3. \\
\hline & -6.5 & & \\
\hline & & & -0.2 \\
\hline & & & \\
\hline & & & \\
\hline & & & \\
\hline & & & \\
\hline & & -2.8 & \\
\hline & & -3.4 & \\
\hline & & -2.6 & \\
\hline & -8.3 & -2.6 & \\
\hline & 06 & & \\
\hline & -12 . & & \\
\hline & & & \\
\hline & & & \\
\hline & & & \\
\hline & & & \\
\hline & & -1.9 & \\
\hline & & -1.0 & \\
\hline & -0.3 & -0.3 & \\
\hline & & -1.7 & \\
\hline & & -0.2 & \\
\hline & -11.0 & -1.4 & 0.3972 \\
\hline & & & -2.4113 \\
\hline & & 0.24235 & -1.1747 \\
\hline & & & -0.69545 \\
\hline & -6.01228 & & -1.05234 \\
\hline & 1.69651 & 1.15846 & -0.16396 \\
\hline
\end{tabular}




$\begin{array}{lccc}\text { BF-DPA }-2270.2785 \text { hartrees } \\ \text { C } & 0.00969 & 1.45060 & -0.26464 \\ \text { C } & 0.80302 & 0.45317 & 0.39483 \\ \text { C } & 0.18200 & -0.59463 & 1.03887 \\ \text { C } & -1.23869 & -0.71355 & 1.05279 \\ \text { C } & -2.03683 & 0.30374 & 0.40648 \\ \text { C } & -1.35594 & 1.37822 & -0.25065 \\ \text { C } & -1.88993 & -1.78080 & 1.69131 \\ \text { C } & -3.28645 & -1.87465 & 1.72075 \\ \text { C } & -4.08453 & -0.85197 & 1.08307 \\ \text { C } & -3.43319 & 0.21092 & 0.43728 \\ \text { C } & 2.83505 & 1.81441 & 0.55173 \\ \text { C } & 3.00691 & -0.61380 & 0.18608 \\ \text { C } & -8.18982 & -3.32872 & 1.66287 \\ \text { C } & -8.30260 & -0.90297 & 2.04572 \\ \text { C } & 2.31321 & 2.72098 & 1.48920 \\ \text { C } & 2.91393 & 3.96826 & 1.66732 \\ \text { C } & 4.04863 & 4.32297 & 0.93321 \\ \text { C } & 4.57571 & 3.41571 & 0.00725 \\ \text { C } & 3.97312 & 2.17518 & -0.19124 \\ \text { C } & -7.72696 & -4.24186 & 0.70134 \\ \text { C } & -8.37066 & -5.46896 & 0.53240 \\ \text { C } & -9.49125 & -5.79699 & 1.30059 \\ \text { C } & -9.95774 & -4.88378 & 2.25301 \\ \text { C } & -9.31129 & -3.66376 & 2.44188 \\ \text { C } & -7.87588 & 0.06834 & 2.97188 \\ \text { C } & -8.60944 & 1.23686 & 3.17113 \\ \text { C } & -9.79373 & 1.43326 & 2.45424 \\ \text { C } & -10.22950 & 0.45620 & 1.53862 \\ \text { C } & -9.49144 & -0.69840 & 1.32077 \\ \text { C } & 4.17373 & -0.79265 & 0.95094 \\ \text { C } & 4.93194 & -1.94096 & 0.77016 \\ \text { C } & 4.53741 & -2.93410 & -0.14662 \\ \text { C } & -6.63378 & -6.10838 & -0.55726 \\ \text { C } & 3.37489 & -2.76204 & -0.90517 \\ \text { C } & 2.62241 & -1.60048 & -0.74311 \\ \text { C } & 5.51386 & -4.02730 & -0.10218 \\ \text { C } & 6.50844 & -3.69920 & 0.84226 \\ \text { C } & 6.22877 & -2.33619 & 1.47290 \\ \text { C } & -11.54489 & 0.87325 & 0.88505 \\ & -11.78651 & 2.23300 & 1.53845 \\ \text { C } & -5.23348 & 2.53894 & 2.45369 \\ \text { C } & -0.80615\end{array}$




$\begin{array}{cccc}\mathrm{C} & -10.77774 & 3.73894 & 3.17092 \\ \mathrm{C} & -11.83549 & 4.63041 & 2.96290 \\ \mathrm{C} & -12.85644 & 4.32676 & 2.05318 \\ \mathrm{C} & -12.83563 & 3.12251 & 1.33525 \\ \mathrm{C} & 7.35703 & -1.33129 & 1.15290 \\ \mathrm{C} & 6.03249 & -2.44794 & 2.99910 \\ \mathrm{C} & -11.40296 & 0.99852 & -0.64600 \\ \mathrm{C} & -12.67204 & -0.12294 & 1.23638 \\ \mathrm{C} & -3.96729 & -2.95387 & 2.37016 \\ \mathrm{C} & -5.33329 & -3.01808 & 2.39862 \\ \mathrm{C} & -6.12589 & -2.00464 & 1.76474 \\ \mathrm{C} & -5.50499 & -0.96039 & 1.11482 \\ \mathrm{H} & 0.51366 & 2.26102 & -0.78059 \\ \mathrm{H} & 0.77866 & -1.35487 & 1.53345 \\ \mathrm{H} & -1.94524 & 2.13756 & -0.75820 \\ \mathrm{H} & -1.29522 & -2.55168 & 2.17641 \\ \mathrm{H} & -4.02806 & 0.98043 & -0.04992 \\ \mathrm{H} & 1.43850 & 2.44535 & 2.06919 \\ \mathrm{H} & 2.49698 & 4.66041 & 2.39332 \\ \mathrm{H} & 4.51559 & 5.29226 & 1.07816 \\ \mathrm{H} & 5.45256 & 3.68110 & -0.57658 \\ \mathrm{H} & 4.37511 & 1.47835 & -0.91955 \\ \mathrm{H} & -6.86671 & -3.98476 & 0.09196 \\ \mathrm{H} & -8.00054 & -6.16516 & -0.21481 \\ \mathrm{H} & -9.99405 & -6.74893 & 1.16034 \\ \mathrm{H} & -10.82329 & -5.12738 & 2.86271 \\ \mathrm{H} & -9.67017 & -2.96139 & 3.18698 \\ \mathrm{H} & -6.95978 & -0.10011 & 3.52753 \\ \mathrm{H} & -8.26384 & 1.97539 & 3.88843 \\ \mathrm{H} & -9.82018 & -1.44740 & 0.60721 \\ \mathrm{H} & 4.46920 & -0.03143 & 1.66630 \\ \mathrm{H} & 3.06218 & -3.51342 & -1.62486 \\ \mathrm{H} & 1.72280 & -1.44793 & -1.32986 \\ \mathrm{H} & 8.33189 & -4.32595 & 1.81384 \\ \mathrm{H} & 8.44040 & -6.47349 & 0.56193 \\ \mathrm{H} & 6.69364 & -7.04998 & -1.09567 \\ \mathrm{H} & 4.80588 & -5.48964 & -1.53274 \\ \mathrm{H} & -9.98573 & 3.97889 & 3.87501 \\ \mathrm{H} & -11.86600 & 5.56781 & 3.51108 \\ \mathrm{H} & -13.67029 & 5.03033 & 1.90308 \\ \mathrm{H} & -13.63074 & 2.89330 & 0.63023 \\ \mathrm{H} & 7.50646 & -1.24555 & 0.07230 \\ \mathrm{H} & 7.11024 & -0.33964 & 1.54682 \\ \mathrm{H} & 8.29993 & -1.65571 & 1.60602 \\ \mathrm{H} & 5.23368 & -3.15642 & 3.23846 \\ \mathrm{H} & 6.95391 & -2.79284 & 3.48067\end{array}$




$\begin{array}{cccc}\mathrm{H} & 5.76889 & -1.47400 & 3.42576 \\ \mathrm{H} & -10.60610 & 1.70126 & -0.90760 \\ \mathrm{H} & -11.16576 & 0.02632 & -1.09160 \\ \mathrm{H} & -12.33752 & 1.35805 & -1.09006 \\ \mathrm{H} & -12.78364 & -0.21708 & 2.32084 \\ \mathrm{H} & -13.62666 & 0.21661 & 0.82099 \\ \mathrm{H} & -12.45152 & -1.11383 & 0.82472 \\ \mathrm{H} & -3.37782 & -3.72253 & 2.86321 \\ \mathrm{H} & -5.83647 & -3.83175 & 2.90997 \\ \mathrm{H} & -6.10246 & -0.19337 & 0.63187 \\ \mathrm{~N} & -7.53787 & -2.08372 & 1.85025 \\ \mathrm{~N} & 2.21586 & 0.55664 & 0.33839\end{array}$

\section{Excited State Calculations BF-DPP}

\footnotetext{
Restricted Singlet Excited State 1:

Excitation energy $=0.1122752747$ hartrees $\quad 3.05516567 \mathrm{eV} \quad 405.82 \mathrm{~nm}$

excitation $\mathrm{X}$ coeff.

$170=>172 \quad-0.13136$

$171 \Rightarrow 172-0.96207$

$171 \Rightarrow 174 \quad-0.18158$

Transition dipole moment (debye):

$$
\mathrm{X}=-0.6260 \quad \mathrm{Y}=0.0954 \quad \mathrm{Z}=-0.1044 \mathrm{Tot}=0.6418
$$

Oscillator strength, $\mathrm{f}=0.0048$

Restricted Singlet Excited State 2:

Excitation energy $=0.1156978287$ hartrees $\quad 3.14829810 \mathrm{eV} \quad 393.81 \mathrm{~nm}$

$$
\begin{aligned}
& \text { excitation X coeff. } \\
& \text {-------- ------- } \\
& 171 \Rightarrow 173 \quad 0.97110 \\
& 171 \Rightarrow 175 \quad 0.16849
\end{aligned}
$$

Transition dipole moment (debye):

$$
\mathrm{X}=3.7135 \quad \mathrm{Y}=4.5269 \quad \mathrm{Z}=2.1538 \mathrm{Tot}=6.2387
$$
}


Oscillator strength, $\mathrm{f}=\quad 0.4647$

Restricted Singlet Excited State 3:

Excitation energy $=0.1237032892$ hartrees $\quad 3.36613777 \mathrm{eV} \quad 368.33 \mathrm{~nm}$

excitation X coeff.

\begin{tabular}{|c|c|}
\hline $170=>172$ & -0.11847 \\
\hline $71=>172$ & -0.17243 \\
\hline $1 \Rightarrow 17$ & \\
\hline $171 \Rightarrow 175$ & 0.16 \\
\hline
\end{tabular}

Transition dipole moment (debye):

$\mathrm{X}=-2.1351 \quad \mathrm{Y}=-0.1273 \quad \mathrm{Z}=-0.5794 \mathrm{Tot}=2.2160$

Oscillator strength, $\mathrm{f}=\quad 0.0627$

Restricted Singlet Excited State 4:

Excitation energy $=0.1277921216$ hartrees $\quad 3.47740056 \mathrm{eV} \quad 356.54 \mathrm{~nm}$

excitation $\mathrm{X}$ coeff.

$---\cdot------------$

$171=>173 \quad 0.18333$

$171=>174 \quad 0.17517$

$171=>175 \quad-0.95145$

Transition dipole moment (debye):

$\mathrm{X}=-4.8315 \mathrm{Y}=1.3507 \mathrm{Z}=1.1060 \mathrm{Tot}=5.1372$

Oscillator strength, $\mathrm{f}=\quad 0.3480$

Restricted Singlet Excited State 5:

Excitation energy $=0.1314409471$ hartrees $\quad 3.57669015 \mathrm{eV} \quad 346.65 \mathrm{~nm}$ excitation X coeff. 


$$
\begin{array}{ll}
170=>174 & -0.10431 \\
170=>176 & 0.12624 \\
171=>176 & 0.96375
\end{array}
$$

Transition dipole moment (debye):

$$
\mathrm{X}=0.1718 \mathrm{Y}=1.9239 \mathrm{Z}=-2.8761 \mathrm{Tot}=3.4645
$$

Oscillator strength, $\mathrm{f}=0.1628$

Restricted Singlet Excited State 6 :

Excitation energy $=0.1366364710$ hartrees $\quad 3.71806755 \mathrm{eV} \quad 333.46 \mathrm{~nm}$

$$
\begin{aligned}
& \text { excitation X coeff. } \\
& \text {----------- --------- } \\
& 170=>172 \quad 0.63748 \\
& 170=>174 \quad 0.19749 \\
& 170 \Rightarrow 175 \quad 0.12552 \\
& 171=>172 \quad-0.14814 \\
& 171=>176 \quad 0.12443 \\
& 171 \Rightarrow 177 \quad 0.65052 \\
& 171=>178 \quad-0.16843
\end{aligned}
$$

Transition dipole moment (debye):

$$
\mathrm{X}=-0.1046 \mathrm{Y}=-0.8667 \mathrm{Z}=-0.4077 \mathrm{Tot}=0.9634
$$

Oscillator strength, $\mathrm{f}=\quad 0.0131$

Restricted Singlet Excited State 7:

Excitation energy $=0.1387036668$ hartrees $\quad 3.77431881 \mathrm{eV} \quad 328.49 \mathrm{~nm}$

excitation $\mathrm{X}$ coeff.

$$
\begin{aligned}
& 170=>172 \quad 0.67796 \\
& 170=>173 \quad-0.15281 \\
& 171=>177 \quad-0.66732
\end{aligned}
$$

Transition dipole moment (debye):

$$
\mathrm{X}=0.2976 \mathrm{Y}=-1.0365 \mathrm{Z}=0.8824 \mathrm{Tot}=1.3934
$$

Oscillator strength, $\mathrm{f}=\quad 0.0278$ 
Restricted Singlet Excited State 8:

Excitation energy $=0.1421683454$ hartrees $\quad 3.86859751 \mathrm{eV} \quad 320.49 \mathrm{~nm}$ excitation X coeff.

\begin{tabular}{|c|c|}
\hline $170=>17$ & 0.12906 \\
\hline $170 \Rightarrow 173$ & 0.95398 \\
\hline $170=>175$ & \\
\hline
\end{tabular}

Transition dipole moment (debye):
$\mathrm{X}=0.502$
$\mathrm{Y}=-2.9724$
$Z=-1.2236 \mathrm{Tot}=$
3.2535

Oscillator strength, $\mathrm{f}=\quad 0.1553$

Restricted Singlet Excited State 9:

Excitation energy $=0.1442294114$ hartrees $\quad 3.92468197 \mathrm{eV} \quad 315.91 \mathrm{~nm}$

excitation $\mathrm{X}$ coeff.

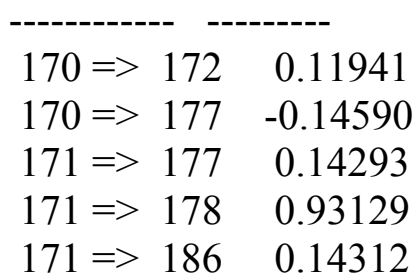

Transition dipole moment (debye):
$\mathrm{X}=0.998$
$\mathrm{Y}=0.5790 \quad \mathrm{Z}=$
0.2117 Tot=
1.1738

Oscillator strength, $\mathrm{f}=\quad 0.0205$

Restricted Singlet Excited State 10:

Excitation energy $=0.1468090524$ hartrees $\quad 3.99487757 \mathrm{eV} \quad 310.36 \mathrm{~nm}$

excitation X coeff.

$171=>179 \quad 0.93429$ 


$$
\begin{array}{rlr}
171 & =>181 & 0.17934 \\
171 & =>182 & -0.16426 \\
171 & =>184 & 0.10044
\end{array}
$$

Transition dipole moment (debye):

$$
\mathrm{X}=-0.1083 \mathrm{Y}=0.3059 \mathrm{Z}=0.0693 \mathrm{Tot}=0.3318
$$

Oscillator strength, $\mathrm{f}=\quad 0.0017$

Restricted Singlet Excited State 11:

Excitation energy $=0.1491912720$ hartrees $\quad 4.05970107 \mathrm{eV} \quad 305.40 \mathrm{~nm}$

$$
\begin{aligned}
& \text { excitation X coeff. } \\
& \text {------------ --------- } \\
& 170=>174 \quad-0.71436 \\
& 170=>176 \quad-0.11707 \\
& 170=>178 \quad-0.11753 \\
& 171 \Rightarrow 177 \quad 0.15025 \\
& 171=>179 \quad-0.14613 \\
& 171=>182-0.57671
\end{aligned}
$$

Transition dipole moment (debye):

$$
\mathrm{X}=0.1153 \mathrm{Y}=0.5282 \quad \mathrm{Z}=0.8282 \mathrm{Tot}=0.9891
$$

Oscillator strength, $\mathrm{f}=\quad 0.0151$

Restricted Singlet Excited State 12:

Excitation energy $=0.1497752840$ hartrees $\quad 4.07559284 \mathrm{eV} \quad 304.21 \mathrm{~nm}$

$$
\begin{aligned}
& \text { excitation X coeff. } \\
& 170 \Rightarrow 174 \quad-0.13863 \\
& 170 \Rightarrow 175 \quad 0.19455 \\
& 171 \Rightarrow 180 \quad-0.93016 \\
& 171 \Rightarrow 182 \quad 0.12026
\end{aligned}
$$

Transition dipole moment (debye):

$$
\mathrm{X}=-0.5612 \quad \mathrm{Y}=-0.1353 \quad \mathrm{Z}=1.3055 \text { Tot }=1.4275
$$

Oscillator strength, $\mathrm{f}=\quad 0.0315$ 
Restricted Singlet Excited State 13:

Excitation energy $=0.1512067145$ hartrees $\quad 4.11454405 \mathrm{eV} \quad 301.33 \mathrm{~nm}$ excitation X coeff.

\begin{tabular}{|c|c|c|}
\hline 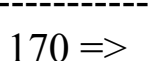 & 172 & \\
\hline & 11 & \\
\hline & 175 & 0.13 \\
\hline & 177 & 0.12 \\
\hline & 180 & -0.1 \\
\hline-7 & 107 & 07 \\
\hline
\end{tabular}

Transition dipole moment (debye):

$X=-0.4978 \quad Y=0.3744 \quad Z=0.7303$ Tot $=0.9599$

Oscillator strength, $\mathrm{f}=\quad 0.0144$

Restricted Singlet Excited State 14:

Excitation energy $=0.1522466517$ hartrees $\quad 4.14284218 \mathrm{eV} \quad 299.27 \mathrm{~nm}$

excitation $\mathrm{X}$ coeff.

\begin{tabular}{|c|c|}
\hline $70=>173$ & \\
\hline & \\
\hline$\Rightarrow 177$ & \\
\hline$\Rightarrow 180$ & \\
\hline $171 \Rightarrow 182$ & \\
\hline $171 \Rightarrow 184$ & \\
\hline
\end{tabular}

Transition dipole moment (debye):

$$
\mathrm{X}=-0.6276 \quad \mathrm{Y}=-1.7061 \quad \mathrm{Z}=-0.6216 \mathrm{Tot}=1.9213
$$

Oscillator strength, $\mathrm{f}=\quad 0.0580$

Restricted Singlet Excited State 15:

Excitation energy $=0.1547984626$ hartrees $\quad 4.21228049 \mathrm{eV} \quad 294.34 \mathrm{~nm}$ 


$\begin{array}{rr}\text { excitation } & \text { X coeff. } \\ -170=>179 & 0.12863 \\ 171=>179 & 0.13341 \\ 171=>181 & -0.90969 \\ 171=>183 & -0.16985 \\ 171=>185 & -0.18017 \\ 171=>188 & 0.11320 \\ 171=>189 & 0.13483\end{array}$

Transition dipole moment (debye):

$$
\mathrm{X}=0.0450 \quad \mathrm{Y}=-0.2972 \quad \mathrm{Z}=0.1225 \text { Tot }=0.3246
$$

Oscillator strength, $\mathrm{f}=\quad 0.0017$

\section{BF-DPB}

Restricted Singlet Excited State 1:

Excitation energy $=0.1162462297$ hartrees $\quad 3.16322085 \mathrm{eV} \quad 391.96 \mathrm{~nm}$ excitation $\mathrm{X}$ coeff.

$$
\begin{array}{rlr}
190 & =>192 & -0.13792 \\
190 & =>194 & 0.11125 \\
191 & =>192 & 0.93418 \\
191 & =>193 & -0.17163 \\
191 & =>194 & 0.21613
\end{array}
$$

Transition dipole moment (debye):

$$
\mathrm{X}=7.5500 \mathrm{Y}=-0.6984 \mathrm{Z}=-3.7171 \text { Tot }=8.4443
$$

Oscillator strength, $\mathrm{f}=\quad 0.8554$

Restricted Singlet Excited State 2:

Excitation energy $=0.1180605412$ hartrees $\quad 3.21259078 \mathrm{eV} \quad 385.93 \mathrm{~nm}$ excitation X coeff. 


$$
\begin{aligned}
& 190=>193 \quad 0.40645 \\
& 191 \Rightarrow 192 \quad 0.18198 \\
& 191=>193 \quad 0.87477
\end{aligned}
$$

Transition dipole moment (debye):

$$
\mathrm{X}=0.6923 \mathrm{Y}=-0.1183 \mathrm{Z}=0.8985 \mathrm{Tot}=1.1405
$$

Oscillator strength, $\mathrm{f}=\quad 0.0158$

Restricted Singlet Excited State 3:

Excitation energy $=0.1247065276$ hartrees $\quad 3.39343727 \mathrm{eV} \quad 365.36 \mathrm{~nm}$

$\begin{array}{ll}\text { excitation } & \text { X coeff. } \\ -------- & ---- \\ 190=>192 & 0.17627 \\ 190=>195 & 0.15479 \\ 191=>192 & -0.14108 \\ 191=>194 & 0.86690 \\ 191=>195 & -0.34755\end{array}$

Transition dipole moment (debye):

$$
\mathrm{X}=3.8550 \mathrm{Y}=0.5622 \quad \mathrm{Z}=2.6511 \mathrm{Tot}=4.7123
$$

\begin{tabular}{|c|c|}
\hline $190=>19$ & 0.12495 \\
\hline $190 \Rightarrow 19$ & -0.28702 \\
\hline $91 \Rightarrow 194$ & $0.348^{\prime}$ \\
\hline $191 \Rightarrow 195$ & 0.852 \\
\hline
\end{tabular}

Oscillator strength, $\mathrm{f}=\quad 0.2858$

Restricted Singlet Excited State 4 :

Excitation energy $=0.1284478633$ hartrees $\quad 3.49524420 \mathrm{eV} \quad 354.72 \mathrm{~nm}$ excitation X coeff.

Transition dipole moment (debye):

$$
\mathrm{X}=2.0018 \mathrm{Y}=-0.6142 \quad \mathrm{Z}=2.4640 \text { Tot }=3.2335
$$

Oscillator strength, $\mathrm{f}=\quad 0.1386$ 
Restricted Singlet Excited State 5:

Excitation energy $=0.1326267281$ hartrees $\quad 3.60895689 \mathrm{eV} \quad 343.55 \mathrm{~nm}$ excitation X coeff.

\begin{tabular}{|c|c|}
\hline $190 \Rightarrow 192$ & \\
\hline $190 \Rightarrow 194$ & 0.359 \\
\hline $191 \Rightarrow 192$ & 0.125 \\
\hline $191 \Rightarrow 194$ & -0.1 \\
\hline
\end{tabular}

Transition dipole moment (debye):

$$
\mathrm{X}=0.1722 \quad \mathrm{Y}=0.0547 \quad \mathrm{Z}=0.7948 \text { Tot }=0.8151
$$

Oscillator strength, $\mathrm{f}=0.0091$

Restricted Singlet Excited State 6:

Excitation energy $=0.1338734846$ hartrees $\quad 3.64288286 \mathrm{eV} \quad 340.35 \mathrm{~nm}$

$$
\begin{aligned}
& \text { excitation X coeff. } \\
& \text { - } \\
& 190 \Rightarrow 193 \quad 0.86099 \\
& 190 \Rightarrow 196 \quad 0.10926 \\
& 191 \Rightarrow 193 \quad-0.42396 \\
& 191 \Rightarrow 196 \quad 0.14004
\end{aligned}
$$

Transition dipole moment (debye):

$$
\mathrm{X}=0.2483 \quad \mathrm{Y}=0.5624 \quad \mathrm{Z}=1.0594 \mathrm{Tot}=1.2249
$$

Oscillator strength, $\mathrm{f}=\quad 0.0207$

Restricted Singlet Excited State 7:

Excitation energy $=0.1354946106$ hartrees $\quad 3.68699595 \mathrm{eV} \quad 336.27 \mathrm{~nm}$ excitation X coeff.

$190=>193 \quad-0.20101$ 


$$
\begin{array}{ll}
190=>195 & -0.10400 \\
190=>196 & 0.36349 \\
191=>196 & 0.86855 \\
191=>197 & 0.14663
\end{array}
$$

Transition dipole moment (debye):

$$
\mathrm{X}=-0.4022 \quad \mathrm{Y}=2.3227 \quad \mathrm{Z}=1.6598 \mathrm{Tot}=2.8830
$$

Oscillator strength, $\mathrm{f}=\quad 0.1162$

Restricted Singlet Excited State 8:

Excitation energy $=0.1396760704$ hartrees $\quad 3.80077926 \mathrm{eV} \quad 326.21 \mathrm{~nm}$

$$
\begin{aligned}
& \text { excitation X coeff. } \\
& 190 \Rightarrow 192 \quad-0.30984 \\
& 190 \Rightarrow 194 \quad 0.72026 \\
& 190 \Rightarrow 197 \quad 0.22947 \\
& 191 \Rightarrow 192 \quad-0.14879 \\
& 191 \Rightarrow 196 \quad-0.12395 \\
& 191 \Rightarrow 197 \quad 0.49592
\end{aligned}
$$

Transition dipole moment (debye):

$$
\mathrm{X}=0.2882 \quad \mathrm{Y}=-1.3289 \quad \mathrm{Z}=-2.8140 \mathrm{Tot}=3.1254
$$

Oscillator strength, $\mathrm{f}=0.1408$

Restricted Singlet Excited State 9:

Excitation energy $=0.1406269028$ hartrees $\quad 3.82665272 \mathrm{eV} \quad 324.00 \mathrm{~nm}$

excitation X coeff.

$$
\begin{aligned}
& 190 \Rightarrow 192 \quad 0.13512 \\
& 190 \Rightarrow 193 \quad-0.11022 \\
& 190 \Rightarrow 194 \quad-0.52234 \\
& 190 \Rightarrow 197 \quad 0.29995 \\
& 191 \Rightarrow 197 \quad 0.72961
\end{aligned}
$$

Transition dipole moment (debye):

$$
X=-1.0187 \quad Y=-1.4059 \quad Z=0.5665 \text { Tot }=1.8263
$$


Oscillator strength, $\mathrm{f}=\quad 0.0484$

Restricted Singlet Excited State 10:

Excitation energy $=0.1448759283$ hartrees $\quad 3.94227459 \mathrm{eV} \quad 314.50 \mathrm{~nm}$

excitation $\mathrm{X}$ coeff.

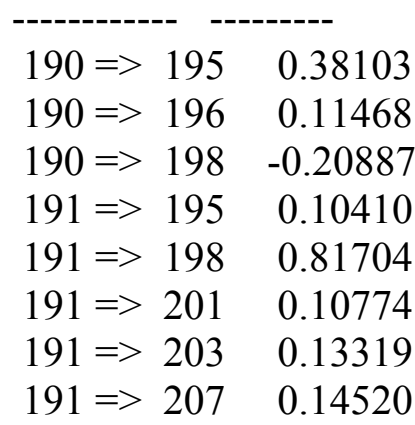

Transition dipole moment (debye):

$\mathrm{X}=0.1629 \mathrm{Y}=0.0878 \quad \mathrm{Z}=0.1050 \mathrm{Tot}=0.2128$

Oscillator strength, $\mathrm{f}=0.0007$

Restricted Singlet Excited State 11:

Excitation energy $=0.1470262702$ hartrees $\quad 4.00078837 \mathrm{eV} \quad 309.90 \mathrm{~nm}$

excitation X coeff.

$$
\begin{aligned}
& 190=>195 \quad 0.81275 \\
& 190=>198 \quad 0.22206 \\
& 191=>195 \quad 0.30280 \\
& 191 \Rightarrow 196 \quad 0.12200 \\
& 191 \Rightarrow 198 \quad-0.35367 \\
& 191=>203 \quad 0.10685
\end{aligned}
$$

Transition dipole moment (debye):

$$
\mathrm{X}=0.1306 \mathrm{Y}=0.2641 \mathrm{Z}=-0.2647 \mathrm{Tot}=0.3961
$$

Oscillator strength, $\mathrm{f}=\quad 0.0024$ 
Restricted Singlet Excited State 12:

Excitation energy $=0.1500773949$ hartrees $\quad 4.08381370 \mathrm{eV} \quad 303.60 \mathrm{~nm}$

excitation X coeff.

\begin{tabular}{|c|c|}
\hline$=>194$ & -0.1 \\
\hline$>196$ & \\
\hline $190 \Rightarrow 197$ & \\
\hline $190=>198$ & \\
\hline $191 \Rightarrow 196$ & -0.1 \\
\hline $191 \Rightarrow 199$ & \\
\hline $191 \Rightarrow 201$ & 0.16 \\
\hline
\end{tabular}

Transition dipole moment (debye):

$\mathrm{X}=-0.6980 \mathrm{Y}=1.2893 \mathrm{Z}=0.3966 \mathrm{Tot}=1.5188$

Oscillator strength, $\mathrm{f}=\quad 0.0357$

Restricted Singlet Excited State 13:

Excitation energy $=0.1511900570$ hartrees $\quad 4.11409077 \mathrm{eV} \quad 301.36 \mathrm{~nm}$

excitation $\mathrm{X}$ coeff.

$$
\begin{array}{rlr}
190 & =>196 & -0.70522 \\
190 & =>197 & -0.18409 \\
190 & =>203 & 0.12455 \\
191 & =>196 & 0.27773 \\
191 & =>198 & 0.16904 \\
191 & =>199 & -0.35687 \\
191 & =>201 & -0.19532 \\
191 & =>203 & -0.36901
\end{array}
$$

Transition dipole moment (debye):

$$
\mathrm{X}=0.5511 \mathrm{Y}=-0.8036 \mathrm{Z}=-1.0830 \mathrm{Tot}=1.4569
$$

Oscillator strength, $\mathrm{f}=0.0331$

Restricted Singlet Excited State 14: 


$$
\begin{aligned}
& \text { Excitation energy }=0.1520462888 \text { hartrees } \\
& \text { excitation X coeff. } \\
& 190=>196 \quad 0.28229 \\
& 190 \Rightarrow 197 \quad-0.26115 \\
& 190 \Rightarrow 201 \quad-0.10954 \\
& 190 \Rightarrow 204 \quad 0.25255 \\
& 191 \Rightarrow 196 \quad-0.18577 \\
& 191 \Rightarrow 197 \quad 0.12039 \\
& 191 \Rightarrow 200 \quad-0.44460 \\
& 191 \Rightarrow 201 \quad-0.48482 \\
& 191 \Rightarrow 202 \quad 0.14833 \\
& 191 \Rightarrow 204 \quad 0.44818
\end{aligned}
$$

Transition dipole moment (debye):

$$
X=0.5316 \quad Y=0.2561 \quad Z=-0.0986 \text { Tot }=0.5983
$$

Oscillator strength, $f=0.0056$

Restricted Singlet Excited State 15:

$$
\begin{aligned}
& \text { Excitation energy }=0.1521746338 \text { hartrees } \quad 4.14088247 \mathrm{eV} \quad 299.41 \mathrm{~nm} \\
& \text { excitation X coeff. } \\
& 190=>195 \quad 0.15638 \\
& 190 \Rightarrow 196 \quad 0.32172 \\
& 190 \Rightarrow 197 \quad 0.10704 \\
& 190 \Rightarrow 202 \quad 0.10119 \\
& 190 \Rightarrow 203 \quad 0.23531 \\
& 191 \Rightarrow 196 \quad-0.12689 \\
& 191 \Rightarrow 199 \quad 0.14707 \\
& 191 \Rightarrow 200 \quad 0.34843 \\
& 191 \Rightarrow 201 \quad-0.25672 \\
& 191 \Rightarrow 202 \quad-0.23168 \\
& 191 \Rightarrow 203 \quad-0.66113 \\
& 191 \Rightarrow 204-0.12132
\end{aligned}
$$

Transition dipole moment (debye):

$$
\mathrm{X}=0.3269 \mathrm{Y}=0.1477 \quad \mathrm{Z}=-0.4612 \mathrm{Tot}=0.5843
$$

Oscillator strength, $\mathrm{f}=\quad 0.0054$ 


\section{BF-DPT}

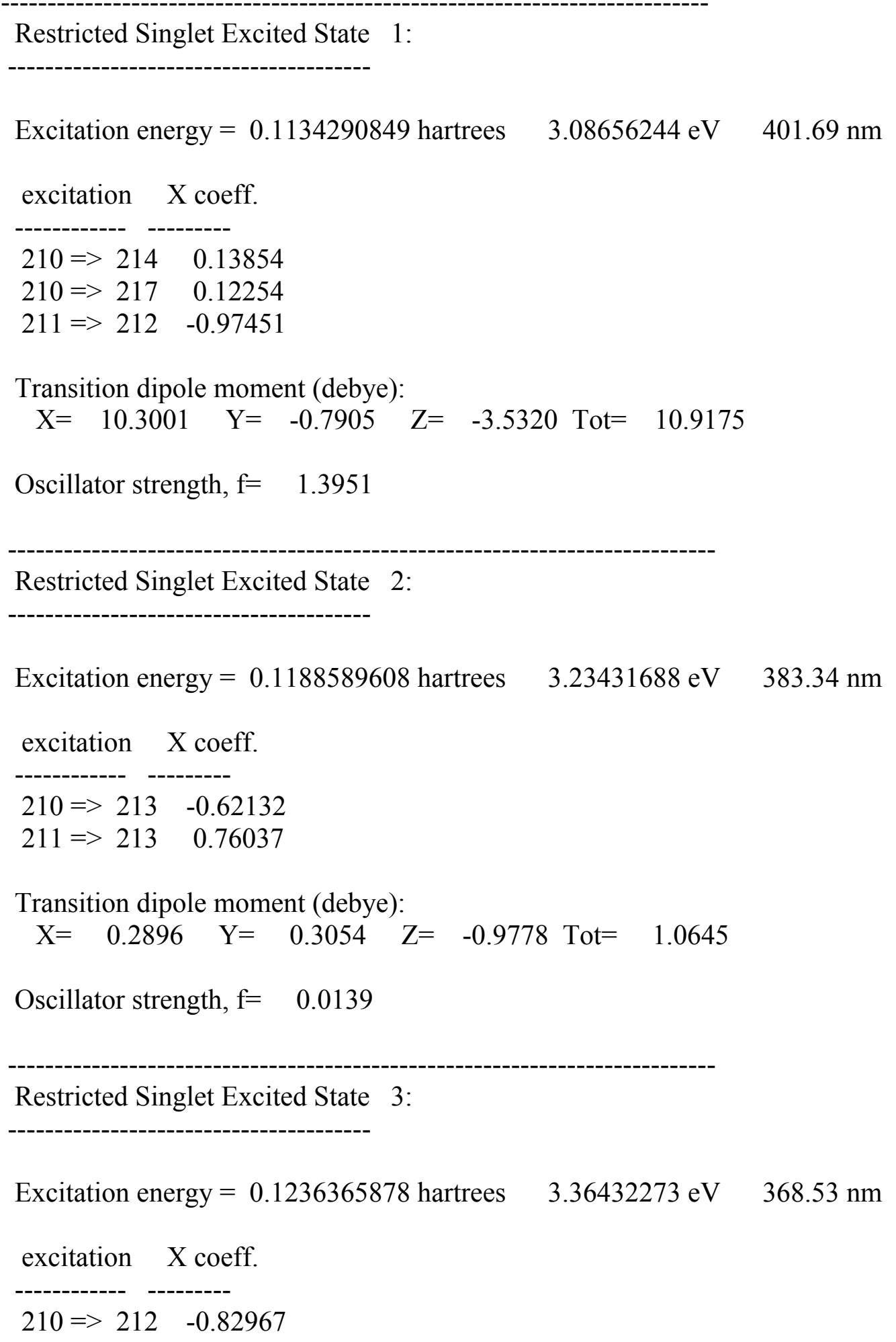




$$
211=>214 \quad 0.50831
$$

$211=215 \quad-0.11540$

Transition dipole moment (debye):

$$
\mathrm{X}=-2.1383 \mathrm{Y}=-0.0161 \mathrm{Z}=-2.2266 \mathrm{Tot}=3.0871
$$

Oscillator strength, $\mathrm{f}=\quad 0.1216$

$$
\text { Restricted Singlet Excited State 4: }
$$

\begin{tabular}{|c|c|}
\hline excitation & X coeff. \\
\hline $210 \Rightarrow 212$ & 0.50743 \\
\hline $210 \Rightarrow 214$ & 0.31962 \\
\hline $210 \Rightarrow 215$ & -0.16171 \\
\hline $211 \Rightarrow 214$ & 0.68522 \\
\hline $211 \Rightarrow 215$ & -0.32873 \\
\hline
\end{tabular}

Excitation energy $=0.1258219900$ hartrees $\quad 3.42379055 \mathrm{eV} \quad 362.13 \mathrm{~nm}$

Transition dipole moment (debye):

$$
\mathrm{X}=-0.2072 \mathrm{Y}=-1.0239 \mathrm{Z}=-2.9585 \mathrm{Tot}=3.1375
$$

Oscillator strength, $f=0.1278$

\section{Restricted Singlet Excited State 5:}

Excitation energy $=0.1287728175$ hartrees $\quad 3.50408665 \mathrm{eV} \quad 353.83 \mathrm{~nm}$

$$
\begin{aligned}
& \text { excitation X coeff. } \\
& 210=>215 \quad-0.39552 \\
& 211=>214 \quad-0.36783 \\
& 211=>215 \quad-0.81224
\end{aligned}
$$

Transition dipole moment (debye):

$$
\mathrm{X}=0.3843 \mathrm{Y}=-0.3184 \mathrm{Z}=2.7334 \mathrm{Tot}=2.7786
$$

Oscillator strength, $\mathrm{f}=0.1026$

Restricted Singlet Excited State 6 : 
Excitation energy $=0.1329462322$ hartrees $\quad 3.61765104 \mathrm{eV} \quad 342.72 \mathrm{~nm}$ excitation $\mathrm{X}$ coeff.

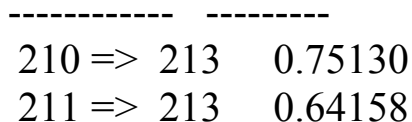

Transition dipole moment (debye):

$$
\mathrm{X}=-0.0694 \mathrm{Y}=0.1534 \mathrm{Z}=0.5623 \mathrm{Tot}=0.5870
$$

Oscillator strength, $\mathrm{f}=\quad 0.0047$

Restricted Singlet Excited State 7:

Excitation energy $=0.1369580619$ hartrees $\quad 3.72681848 \mathrm{eV} \quad 332.68 \mathrm{~nm}$

\begin{tabular}{|c|c|}
\hline excitation & coeff. \\
\hline $210 \Rightarrow 21$ & -0.1 \\
\hline $210=>21 ?$ & 0.12745 \\
\hline $210=>21$ & 0.29403 \\
\hline $210 \Rightarrow 210$ & 0.55073 \\
\hline $210=>21^{\prime}$ & -0.12234 \\
\hline $211 \Rightarrow 21$ & -0.10141 \\
\hline $211 \Rightarrow 210$ & -0.718 \\
\hline
\end{tabular}

Transition dipole moment (debye):

$\mathrm{X}=-0.0330 \quad \mathrm{Y}=-3.1174 \quad \mathrm{Z}=-2.2504 \mathrm{Tot}=3.8450$

Oscillator strength, $\mathrm{f}=\quad 0.2089$

Restricted Singlet Excited State 8:

Excitation energy $=0.1382850401$ hartrees $\quad 3.76292740 \mathrm{eV} \quad 329.49 \mathrm{~nm}$

excitation $\mathrm{X}$ coeff.

$210=>212 \quad 0.13049$

$210=>214 \quad-0.81510$

$210=>216 \quad 0.18282$ 


$$
\begin{array}{rlr}
210 & =>217 & -0.15999 \\
211 & =>212 & -0.11470 \\
211 & =>214 & 0.30161 \\
211 & =>216 & -0.19763 \\
211 & =>217 & 0.28947
\end{array}
$$

Transition dipole moment (debye):

$$
\mathrm{X}=-1.2422 \mathrm{Y}=-0.7884 \mathrm{Z}=1.4527 \mathrm{Tot}=2.0676
$$

Oscillator strength, $\mathrm{f}=\quad 0.0610$

Restricted Singlet Excited State 9:

Excitation energy $=0.1406798786$ hartrees $\quad 3.82809427 \mathrm{eV} \quad 323.88 \mathrm{~nm}$

excitation X coeff.

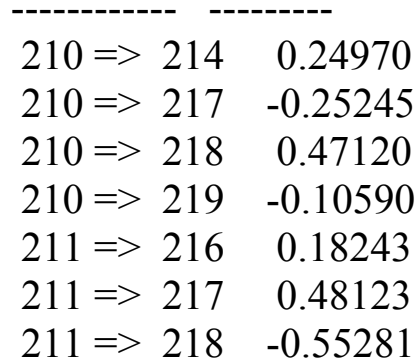

\begin{tabular}{|c|c|}
\hline excitation & X coeff. \\
\hline$>214$ & -0.1 \\
\hline$=>215$ & 082 \\
\hline $210 \Rightarrow 216$ & -0.15514 \\
\hline $210 \Rightarrow 217$ & 11308 \\
\hline $210=>219$ & -0.13654 \\
\hline $211 \Rightarrow 21$ & -0.1509 \\
\hline
\end{tabular}

Transition dipole moment (debye):

$$
\mathrm{X}=1.0959 \mathrm{Y}=-0.1031 \mathrm{Z}=-0.2247 \text { Tot }=1.1235
$$

Oscillator strength, $\mathrm{f}=\quad 0.0183$

Restricted Singlet Excited State 10:

Excitation energy $=0.1435766348$ hartrees $\quad 3.90691902 \mathrm{eV} \quad 317.35 \mathrm{~nm}$ 
$211=>217 \quad-0.66678$

$211=>218-0.53150$

$211=>219 \quad-0.22585$

$211=>220 \quad-0.10765$

Transition dipole moment (debye):

$$
\mathrm{X}=0.1365 \quad \mathrm{Y}=0.2738 \quad \mathrm{Z}=-0.4594 \text { Tot }=0.5519
$$

Oscillator strength, $\mathrm{f}=\quad 0.0045$

Restricted Singlet Excited State 11:

Excitation energy $=0.1458049700$ hartrees $\quad 3.96755510 \mathrm{eV} \quad 312.50 \mathrm{~nm}$

$$
\begin{aligned}
& \text { excitation X coeff. } \\
& 210 \Rightarrow 215 \quad-0.40656 \\
& 210 \Rightarrow 217 \quad-0.10390 \\
& 210 \Rightarrow 218 \quad 0.21702 \\
& 210 \Rightarrow 220 \quad 0.14622 \\
& 211 \Rightarrow 215 \quad 0.18675 \\
& 211 \Rightarrow 217 \quad-0.27273 \\
& 211 \Rightarrow 219 \quad 0.73634
\end{aligned}
$$

Transition dipole moment (debye):

$$
\mathrm{X}=0.0619 \quad \mathrm{Y}=-0.1164 \quad \mathrm{Z}=-0.0927 \mathrm{Tot}=0.1612
$$

Oscillator strength, $\mathrm{f}=\quad 0.0004$

Restricted Singlet Excited State 12:

$$
\begin{aligned}
& \text { Excitation energy }=0.1470902320 \text { hartrees } \quad 4.00252886 \mathrm{eV} \quad 309.76 \mathrm{~nm} \\
& \begin{array}{rll}
\text { excitation } & \text { X coeff. } \\
\hline 210 & =>215 & 0.66848 \\
210 & \Rightarrow 217 & 0.12328 \\
210 & \Rightarrow 218 & 0.11231 \\
210 & \Rightarrow 219 & 0.28387 \\
210 & \Rightarrow 220 & 0.10695 \\
211 & =>215 & -0.35401 \\
211 & =217 & -0.12142
\end{array}
\end{aligned}
$$


$211=>219 \quad 0.34259$

$211 \Rightarrow 220 \quad 0.37451$

Transition dipole moment (debye):
$\mathrm{X}=0.214$
$\mathrm{Y}=0.4780$
$Z=-0.0572$ Tot
0.5268

Oscillator strength, $\mathrm{f}=\quad 0.0042$

Restricted Singlet Excited State 13:

Excitation energy $=0.1490595558$ hartrees $\quad 4.05611689 \mathrm{eV} \quad 305.67 \mathrm{~nm}$

excitation $\mathrm{X}$ coeff.

\begin{tabular}{|c|c|}
\hline $201 \Rightarrow>212$ & 0.10959 \\
\hline $210 \Rightarrow 215$ & -0.3 \\
\hline$=>217$ & 0.2 \\
\hline$=>219$ & \\
\hline $210 \Rightarrow 224$ & -0.1 \\
\hline $211=>215$ & 0.15 \\
\hline $211 \Rightarrow 218$ & -0.25811 \\
\hline $211 \Rightarrow 219$ & -0.183 \\
\hline $211 \Rightarrow 220$ & $0.746^{\circ}$ \\
\hline
\end{tabular}

Transition dipole moment (debye):

$\mathrm{X}=-0.0880 \mathrm{Y}=0.6563 \mathrm{Z}=0.2395 \mathrm{Tot}=0.7041$

Oscillator strength, $\mathrm{f}=0.0076$

Restricted Singlet Excited State 14:

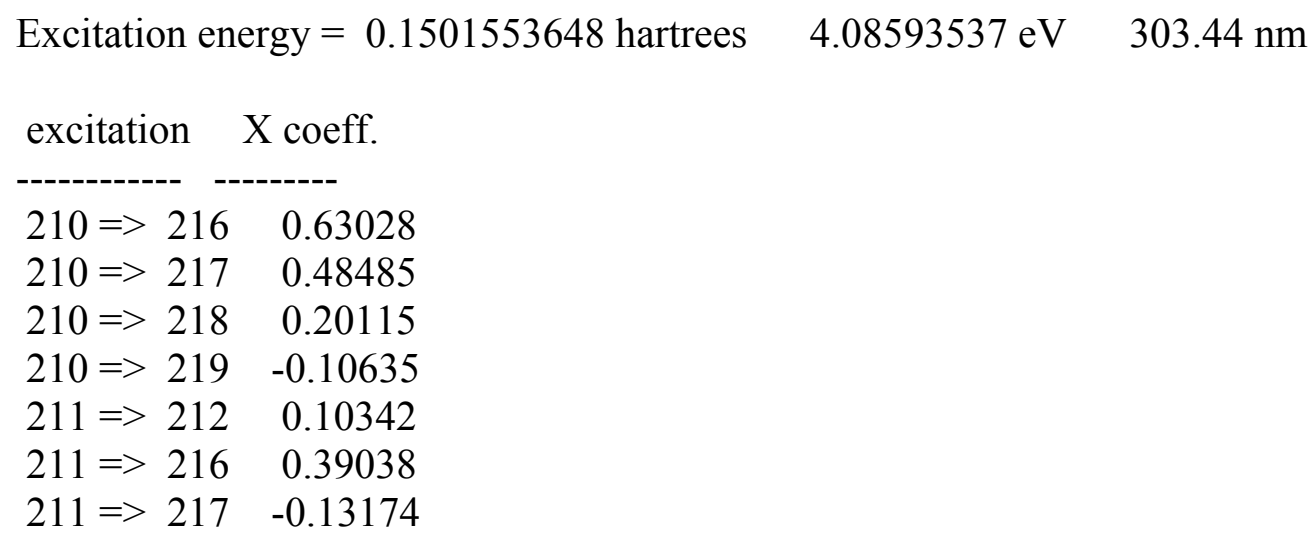


$211=>218-0.13639$

$211 \Rightarrow 220-0.20494$

$211=>221 \quad 0.10939$

Transition dipole moment (debye):

$$
\mathrm{X}=1.8217 \mathrm{Y}=-0.2432 \mathrm{Z}=0.2732 \mathrm{Tot}=1.8581
$$

Oscillator strength, $\mathrm{f}=0.0535$

Restricted Singlet Excited State 15:

Excitation energy $=0.1516641125$ hartrees $\quad 4.12699048 \mathrm{eV} \quad 300.42 \mathrm{~nm}$

\begin{tabular}{|c|c|}
\hline Cc & \\
\hline$>216$ & \\
\hline$\Rightarrow>217$ & -0.62067 \\
\hline$\Rightarrow 218$ & 085 \\
\hline$=>219$ & 0.15626 \\
\hline$\Rightarrow 216$ & 233 \\
\hline $211 \Rightarrow 217$ & -0.17176 \\
\hline $211 \Rightarrow 220$ & 0.17141 \\
\hline $211 \Rightarrow 221$ & -0.20008 \\
\hline $211 \Rightarrow 226$ & -0.12044 \\
\hline
\end{tabular}

Transition dipole moment (debye):

$$
\mathrm{X}=-1.7027 \mathrm{Y}=-0.8703 \mathrm{Z}=-0.8565 \mathrm{Tot}=2.0953
$$

Oscillator strength, $\mathrm{f}=\quad 0.0687$

\section{BF-DPN}

Restricted Singlet Excited State 1:

\footnotetext{
Excitation energy $=0.1055569651$ hartrees $\quad 2.87235116 \mathrm{eV} \quad 431.65 \mathrm{~nm}$

excitation $\mathrm{X}$ coeff.

$184=>185 \quad-0.98034$

$184=>186 \quad-0.10050$
} 
Transition dipole moment (debye):

$$
\mathrm{X}=-4.9872 \quad \mathrm{Y}=1.2782 \quad \mathrm{Z}=-1.7417 \text { Tot }=5.4350
$$

Oscillator strength, $\mathrm{f}=0.3218$

Restricted Singlet Excited State 2:

Excitation energy $=0.1158233488$ hartrees $\quad 3.15171368 \mathrm{eV} \quad 393.39 \mathrm{~nm}$

excitation X coeff.

$$
\begin{array}{rlr}
183 & =>187 & -0.15251 \\
184 & \Rightarrow 185 & -0.10849 \\
184 & =>186 & 0.94893 \\
184 & =188 & 0.19951
\end{array}
$$

Transition dipole moment (debye):

$$
X=-5.8050 \quad Y=-0.3417 \quad Z=-3.9083 \quad \text { Tot }=7.0064
$$

Oscillator strength, $\mathrm{f}=\quad 0.5867$

Restricted Singlet Excited State 3:

Excitation energy $=0.1206017725$ hartrees $\quad 3.28174120 \mathrm{eV} \quad 377.80 \mathrm{~nm}$

$$
\begin{aligned}
& \text { excitation X coeff. } \\
& 183 \Rightarrow 185 \quad 0.23987 \\
& 183 \Rightarrow 186 \quad 0.19711 \\
& 184 \Rightarrow 187 \quad-0.92649 \\
& 184 \Rightarrow 189 \quad 0.13167
\end{aligned}
$$

Transition dipole moment (debye):

$$
\mathrm{X}=0.8365 \quad \mathrm{Y}=4.1042 \quad \mathrm{Z}=-1.4699 \mathrm{Tot}=4.4391
$$

Oscillator strength, $\mathrm{f}=\quad 0.2452$

Restricted Singlet Excited State 4: 
Excitation energy $=0.1232111224$ hartrees $\quad 3.35274522 \mathrm{eV} \quad 369.80 \mathrm{~nm}$

\begin{tabular}{|c|c|}
\hline excitation & X coeff. \\
\hline $183 \Rightarrow 185$ & -0.88245 \\
\hline $183 \Rightarrow 186$ & -0.12390 \\
\hline $184 \Rightarrow 187$ & -0.29296 \\
\hline $184 \Rightarrow 189$ & -0.30596 \\
\hline
\end{tabular}

Transition dipole moment (debye):

$$
\mathrm{X}=0.2317 \quad \mathrm{Y}=2.1541 \quad \mathrm{Z}=-0.3284 \mathrm{Tot}=2.1913
$$

Oscillator strength, $\mathrm{f}=\quad 0.0611$

Restricted Singlet Excited State 5:

Excitation energy $=0.1277546550$ hartrees $\quad 3.47638104 \mathrm{eV} \quad 356.65 \mathrm{~nm}$ excitation X coeff.

$-----------$

$184=>186 \quad-0.20280$

$184 \Rightarrow 188 \quad 0.94785$

Transition dipole moment (debye):

$$
\mathrm{X}=-3.4834 \quad \mathrm{Y}=0.6721 \quad \mathrm{Z}=2.1726 \mathrm{Tot}=4.1601
$$

Oscillator strength, $\mathrm{f}=0.2282$

Restricted Singlet Excited State 6:

Excitation energy $=0.1306225896$ hartrees $\quad 3.55442151 \mathrm{eV} \quad 348.82 \mathrm{~nm}$

excitation $\mathrm{X}$ coeff.

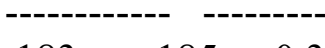

$183 \Rightarrow 185 \quad-0.36571$

$183 \Rightarrow 186 \quad 0.12492$

$183 \Rightarrow 188 \quad 0.14934$

$183 \Rightarrow 190 \quad-0.13246$

$184 \Rightarrow 189 \quad 0.87996$

Transition dipole moment (debye):

$$
\mathrm{X}=0.1835 \quad \mathrm{Y}=-0.5009 \quad \mathrm{Z}=-1.0493 \quad \mathrm{Tot}=1.1771
$$


Oscillator strength, $\mathrm{f}=\quad 0.0187$

Restricted Singlet Excited State 7:

Excitation energy $=0.1373016638$ hartrees $\quad 3.73616837 \mathrm{eV} \quad 331.85 \mathrm{~nm}$

excitation X coeff.

\begin{tabular}{|c|c|c|}
\hline $183=>$ & 185 & 0.10119 \\
\hline $183=>$ & 186 & -0.81266 \\
\hline $183=>$ & 188 & -0.15366 \\
\hline $83=>$ & 189 & -0.16261 \\
\hline $4=>$ & 189 & 0.18512 \\
\hline $184=>$ & 190 & $0.4336^{\prime}$ \\
\hline
\end{tabular}

Transition dipole moment (debye):
$\mathrm{X}=-0.9099$
$\mathrm{Y}=-2.3874$
$\mathrm{Z}=1.4164 \mathrm{Tot}=$
2.9212

Oscillator strength, $\mathrm{f}=\quad 0.1209$

Restricted Singlet Excited State 8:

Excitation energy $=0.1373384322$ hartrees $\quad 3.73716889 \mathrm{eV} \quad 331.76 \mathrm{~nm}$

excitation X coeff.

\begin{tabular}{|c|c|c|}
\hline $183=>$ & 186 & -0.4 \\
\hline & 187 & \\
\hline & 189 & \\
\hline & 187 & -0.1 \\
\hline 24 & 190 & -0.74 \\
\hline $184 \Rightarrow$ & 192 & -0.21 \\
\hline
\end{tabular}

Transition dipole moment (debye):

$\mathrm{X}=0.3813 \mathrm{Y}=-1.1455 \mathrm{Z}=0.4492 \mathrm{Tot}=1.2881$

Oscillator strength, $\mathrm{f}=\quad 0.0235$

Restricted Singlet Excited State 9: 
Excitation energy $=0.1405133697$ hartrees $\quad 3.82356333 \mathrm{eV} \quad 324.26 \mathrm{~nm}$

excitation X coeff.

$--------$

$183=>187 \quad 0.95705$

$184 \Rightarrow 186 \quad 0.16995$

$184 \Rightarrow 190 \quad-0.15870$

Transition dipole moment (debye):

$$
\mathrm{X}=1.9268 \mathrm{Y}=0.9944 \quad \mathrm{Z}=2.9162 \mathrm{Tot}=3.6340
$$

Oscillator strength, $\mathrm{f}=\quad 0.1915$

Restricted Singlet Excited State 10:

Excitation energy $=0.1437460443$ hartrees $\quad 3.91152888 \mathrm{eV} \quad 316.97 \mathrm{~nm}$

\begin{tabular}{|c|c|}
\hline excitation & X coeff. \\
\hline $183 \Rightarrow 188$ & 0.34372 \\
\hline $183 \Rightarrow 191$ & -0.11025 \\
\hline $183 \Rightarrow 192$ & 0.14734 \\
\hline $184 \Rightarrow 190$ & -0.10429 \\
\hline $184 \Rightarrow 191$ & -0.81202 \\
\hline $184 \Rightarrow 192$ & 0.32923 \\
\hline $184 \Rightarrow 199$ & 0.10548 \\
\hline
\end{tabular}

Transition dipole moment (debye):

$\mathrm{X}=0.6640 \quad \mathrm{Y}=-1.1892 \quad \mathrm{Z}=0.7704 \mathrm{Tot}=1.5648$

Oscillator strength, $f=0.0363$

Restricted Singlet Excited State 11:

Excitation energy $=0.1450902114$ hartrees $\quad 3.94810553 \mathrm{eV} \quad 314.03 \mathrm{~nm}$

excitation X coeff.

$183=>186 \quad 0.10019$

$183 \Rightarrow 188 \quad-0.21504$

$183 \Rightarrow 190 \quad-0.13337$ 


$$
\begin{aligned}
& 183=>191 \quad 0.17034 \\
& 183=>192 \quad 0.14588 \\
& 184 \Rightarrow 190 \quad 0.13829 \\
& 184=>191 \quad-0.42939 \\
& 184=>192-0.74421 \\
& 184=>193 \quad 0.19232 \\
& 184=>198 \quad 0.10647
\end{aligned}
$$

Transition dipole moment (debye):

$$
\mathrm{X}=0.9190 \quad \mathrm{Y}=0.8206 \quad \mathrm{Z}=0.0454 \mathrm{Tot}=1.2329
$$

Oscillator strength, $\mathrm{f}=0.0228$

Restricted Singlet Excited State 12:

Excitation energy $=0.1467456714$ hartrees $\quad 3.99315289 \mathrm{eV} \quad 310.49 \mathrm{~nm}$

$$
\begin{aligned}
& \text { excitation X coeff. } \\
& \text {------------- ---------- } \\
& 183 \Rightarrow 188 \quad-0.70193 \\
& 183 \Rightarrow 190 \quad-0.13463 \\
& 183 \Rightarrow 191 \quad-0.12769 \\
& 184 \Rightarrow 189 \quad 0.11382 \\
& 184 \Rightarrow 190 \quad-0.19967 \\
& 184 \Rightarrow 191 \quad-0.12094 \\
& 184 \Rightarrow 192 \quad 0.37032 \\
& 184 \Rightarrow 193 \quad 0.43847 \\
& 184 \Rightarrow 194 \quad 0.13319
\end{aligned}
$$

Transition dipole moment (debye):

$$
\mathrm{X}=0.2440 \mathrm{Y}=1.4881 \quad \mathrm{Z}=-0.5020 \mathrm{Tot}=1.5893
$$

Oscillator strength, $f=0.0383$

Restricted Singlet Excited State 13:

$$
\begin{aligned}
& \text { Excitation energy }=0.1489698055 \text { hartrees } \quad 4.05367466 \mathrm{eV} \quad 305.86 \mathrm{~nm} \\
& \text { excitation X coeff. } \\
& 183 \Rightarrow 195 \quad-0.14972 \\
& 184 \Rightarrow 193 \quad 0.31443
\end{aligned}
$$




$$
\begin{aligned}
& 184=>194 \quad-0.89296 \\
& 184=>196 \quad 0.10200 \\
& 184=>198 \quad-0.11081
\end{aligned}
$$

Transition dipole moment (debye):

$$
\mathrm{X}=-0.2194 \mathrm{Y}=-0.0678 \quad \mathrm{Z}=-0.1208 \text { Tot }=0.2595
$$

Oscillator strength, $\mathrm{f}=\quad 0.0010$

Restricted Singlet Excited State 14:

Excitation energy $=0.1504480507$ hartrees $\quad 4.09389975 \mathrm{eV} \quad 302.85 \mathrm{~nm}$

$$
\begin{aligned}
& \text { excitation X coeff. } \\
& \text {-------- ------ } \\
& 183=>186 \quad 0.11440 \\
& 183=>188 \quad-0.50014 \\
& 184=>191 \quad-0.17596 \\
& 184=>193 \quad-0.76295 \\
& 184=>194 \quad-0.26302
\end{aligned}
$$

Transition dipole moment (debye):

$$
\mathrm{X}=-0.0365 \mathrm{Y}=0.5033 \mathrm{Z}=-0.5265 \mathrm{Tot}=0.7293
$$

Oscillator strength, $\mathrm{f}=\quad 0.0083$

Restricted Singlet Excited State 15:

Excitation energy $=0.1518624672$ hartrees $\quad 4.13238799 \mathrm{eV} \quad 300.03 \mathrm{~nm}$

$$
\begin{aligned}
& \text { excitation X coeff. } \\
& 182=>185 \quad 0.22977 \\
& 183=>189 \quad-0.87375 \\
& 184=>188 \quad 0.11914 \\
& 184=>190 \quad-0.28990 \\
& 184=>192 \quad-0.14641
\end{aligned}
$$

Transition dipole moment (debye):

$$
\mathrm{X}=-0.9821 \mathrm{Y}=-0.4412 \mathrm{Z}=-0.3929 \mathrm{Tot}=1.1461
$$

Oscillator strength, $\mathrm{f}=0.0206$ 


\section{BF-DPA}

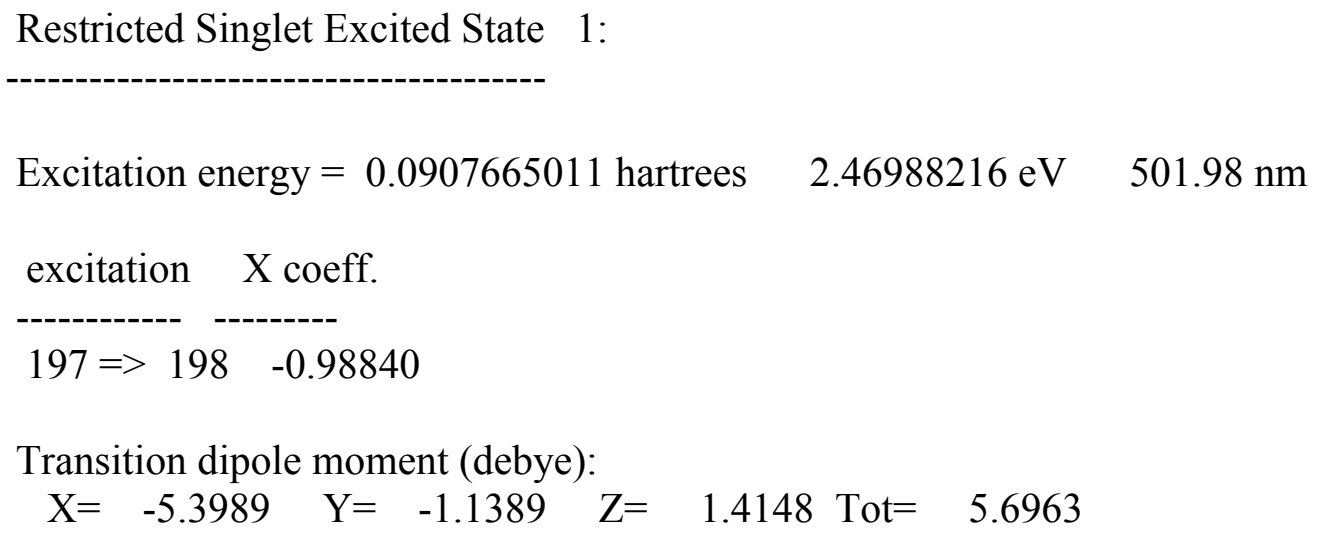

Oscillator strength, $\mathrm{f}=0.3039$

Restricted Singlet Excited State 2:

Excitation energy $=0.1066201092$ hartrees $\quad 2.90128079 \mathrm{eV} \quad 427.34 \mathrm{~nm}$ excitation $\mathrm{X}$ coeff.

$196=>198 \quad-0.98945$

Transition dipole moment (debye):

$$
\mathrm{X}=0.0585 \mathrm{Y}=0.0114 \mathrm{Z}=0.0329 \mathrm{Tot}=0.0681
$$

Oscillator strength, $\mathrm{f}=\quad 0.0001$

Restricted Singlet Excited State 3:

Excitation energy $=0.1152476736$ hartrees $\quad 3.13604876 \mathrm{eV} \quad 395.35 \mathrm{~nm}$ excitation $\mathrm{X}$ coeff.

$195=>198 \quad-0.19571$

$196=>200 \quad 0.17599$

$197=>199 \quad-0.92089$ 


$$
197=>201 \quad-0.23775
$$

Transition dipole moment (debye):

$$
\mathrm{X}=6.4388 \quad \mathrm{Y}=-3.3601 \quad \mathrm{Z}=-0.5988 \text { Tot }=7.2875
$$

Oscillator strength, $f=0.6316$

Restricted Singlet Excited State 4:

Excitation energy $=0.1190317281$ hartrees $\quad 3.23901812 \mathrm{eV} \quad 382.78 \mathrm{~nm}$

excitation X coeff.

$$
\begin{array}{rrr}
196 & =>199 & -0.20682 \\
197 & =>200 & 0.96541
\end{array}
$$

Transition dipole moment (debye):

$$
\mathrm{X}=-0.1440 \quad \mathrm{Y}=0.0120 \quad \mathrm{Z}=-0.0661 \quad \mathrm{Tot}=0.1589
$$

Oscillator strength, $\mathrm{f}=\quad 0.0003$

Restricted Singlet Excited State 5:

Excitation energy $=0.1233016777$ hartrees $\quad 3.35520936 \mathrm{eV} \quad 369.53 \mathrm{~nm}$

$$
\begin{aligned}
& \text { excitation X coeff. } \\
& 194 \Rightarrow 198 \quad-0.13996 \\
& 195 \Rightarrow 198 \quad-0.73433 \\
& 196 \Rightarrow 200 \quad-0.15065 \\
& 197 \Rightarrow 199 \quad 0.29103 \\
& 197 \Rightarrow 201 \quad-0.52039 \\
& 197 \Rightarrow 203 \quad-0.18206
\end{aligned}
$$

Transition dipole moment (debye):

$$
\mathrm{X}=-2.7453 \quad \mathrm{Y}=3.9320 \quad \mathrm{Z}=-0.0310 \mathrm{Tot}=4.7956
$$

Oscillator strength, $f=0.2926$

Restricted Singlet Excited State 6: 
Excitation energy $=0.1273713473$ hartrees $\quad 3.46595071 \mathrm{eV} \quad 357.72 \mathrm{~nm}$

excitation $\mathrm{X}$ coeff.

$196 \Rightarrow 199 \quad-0.13156$

$196 \Rightarrow 201 \quad-0.16745$

$196 \Rightarrow 203 \quad 0.18106$

$197 \Rightarrow 202 \quad-0.93869$

Transition dipole moment (debye):

$\mathrm{X}=-0.0369 \quad \mathrm{Y}=0.0308 \quad \mathrm{Z}=0.0147$ Tot $=0.0503$

Oscillator strength, $\mathrm{f}=\quad 0.0000$

Restricted Singlet Excited State 7:

Excitation energy $=0.1290094028$ hartrees $\quad 3.51052446 \mathrm{eV} \quad 353.18 \mathrm{~nm}$

excitation X coeff.

\begin{tabular}{|c|c|}
\hline $194 \Rightarrow 198$ & -0.1257 \\
\hline$\Rightarrow 198$ & 0.584 \\
\hline$\Rightarrow 204$ & \\
\hline $197 \Rightarrow 201$ & \\
\hline
\end{tabular}

Transition dipole moment (debye):
$\mathrm{X}=6.5797$
$\mathrm{Y}=$
$2.3875 \quad \mathrm{Z}=$
0.1337 Tot $=7.0008$

Oscillator strength, $\mathrm{f}=\quad 0.6525$

Restricted Singlet Excited State 8:

Excitation energy $=0.1330698203$ hartrees $\quad 3.62101405 \mathrm{eV} \quad 342.40 \mathrm{~nm}$

excitation X coeff.

$195=>198 \quad-0.17213$

$196 \Rightarrow 202 \quad-0.40047$

$197 \Rightarrow 203 \quad 0.85806$

$197=>205 \quad 0.11097$ 
Transition dipole moment (debye):

$$
X=-3.0272 \quad Y=0.3977 \quad Z=0.7439 \text { Tot }=3.1425
$$

Oscillator strength, $\mathrm{f}=0.1356$

Restricted Singlet Excited State 9:

Excitation energy $=0.1358040953$ hartrees $\quad 3.69541745 \mathrm{eV} \quad 335.51 \mathrm{~nm}$

excitation X coeff.

$195 \Rightarrow 200 \quad-0.10405$

$196 \Rightarrow 199 \quad 0.93869$

$196 \Rightarrow 201 \quad 0.12505$

$197 \Rightarrow 200 \quad 0.19317$

$197=>202 \quad-0.17048$

Transition dipole moment (debye):

$$
\mathrm{X}=0.0145 \quad \mathrm{Y}=0.0373 \quad \mathrm{Z}=0.0648 \text { Tot }=0.0761
$$

Oscillator strength, $\mathrm{f}=\quad 0.0001$

Restricted Singlet Excited State 10:

\begin{tabular}{|c|c|}
\hline ctit & X coeff. \\
\hline & \\
\hline$>1 S$ & \\
\hline$\Rightarrow 2$ & \\
\hline $97 \stackrel{>}{9}$ & \\
\hline $97=>20$ & \\
\hline
\end{tabular}

Excitation energy $=0.1377444621$ hartrees $\quad 3.74821752 \mathrm{eV} \quad 330.78 \mathrm{~nm}$

Transition dipole moment (debye):

$$
\mathrm{X}=-2.5698 \quad \mathrm{Y}=3.7512 \quad \mathrm{Z}=0.3162 \text { Tot }=4.5580
$$

Oscillator strength, $\mathrm{f}=\quad 0.2953$

Restricted Singlet Excited State 11: 
Excitation energy $=0.1428616064$ hartrees $\quad 3.88746210 \mathrm{eV} \quad 318.93 \mathrm{~nm}$

excitation X coeff.

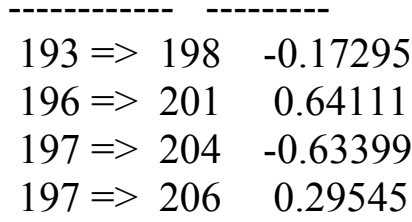

Transition dipole moment (debye):

$$
\mathrm{X}=-0.0518 \mathrm{Y}=0.0070 \quad \mathrm{Z}=0.0315 \text { Tot }=0.0610
$$

Oscillator strength, $\mathrm{f}=\quad 0.0001$

Restricted Singlet Excited State 12:

Excitation energy $=0.1438716816$ hartrees $\quad 3.91494765 \mathrm{eV} \quad 316.69 \mathrm{~nm}$

excitation X coeff.

\begin{tabular}{|c|c|}
\hline $196=>199$ & 0.11890 \\
\hline $196=>201$ & -0.31691 \\
\hline $196=>203$ & -0.22232 \\
\hline $196=>205$ & 0.23000 \\
\hline $197 \Rightarrow 204$ & -0.62871 \\
\hline $197=>206$ & -0.56819 \\
\hline $97=>209$ & 0.10257 \\
\hline $97 \Rightarrow 215$ & -0.10870 \\
\hline
\end{tabular}

Transition dipole moment (debye):

$$
\mathrm{X}=0.0297 \mathrm{Y}=-0.0053 \mathrm{Z}=0.0059 \text { Tot }=0.0307
$$

Oscillator strength, $\mathrm{f}=\quad 0.0000$

Restricted Singlet Excited State 13:

Excitation energy $=0.1443420717$ hartrees $\quad 3.92774761 \mathrm{eV} \quad 315.66 \mathrm{~nm}$

excitation $\mathrm{X}$ coeff.

$188=>198 \quad 0.10340$ 


$$
\begin{array}{rlr}
194 & \Rightarrow>198 & 0.82828 \\
195 & \Rightarrow>199 & -0.28212 \\
195 & \Rightarrow>201 & -0.22961 \\
195 & \Rightarrow 203 & -0.11365 \\
196 & \Rightarrow 200 & -0.16338 \\
196 & \Rightarrow 202 & 0.15231 \\
197 & \Rightarrow 201 & -0.16770 \\
197 & \Rightarrow 213 & -0.11920
\end{array}
$$

Transition dipole moment (debye):

$$
\mathrm{X}=3.2641 \quad \mathrm{Y}=2.2911 \quad \mathrm{Z}=-0.1938 \text { Tot }=3.9926
$$

Oscillator strength, $\mathrm{f}=\quad 0.2374$

Restricted Singlet Excited State 14:

Excitation energy $=0.1446614675$ hartrees $\quad 3.93643882 \mathrm{eV} \quad 314.97 \mathrm{~nm}$

$$
\begin{aligned}
& \text { excitation X coeff. } \\
& 196 \Rightarrow 202 \quad-0.11340 \\
& 196 \Rightarrow 204 \quad-0.21447 \\
& 196 \Rightarrow 206 \quad-0.20542 \\
& 197 \Rightarrow 203 \quad-0.15520 \\
& 197 \Rightarrow 205 \quad 0.89253 \\
& 197 \Rightarrow 213 \quad-0.12308
\end{aligned}
$$

Transition dipole moment (debye):

$$
\mathrm{X}=-1.3364 \quad \mathrm{Y}=0.3242 \quad \mathrm{Z}=0.5814 \mathrm{Tot}=1.4930
$$

Oscillator strength, $\mathrm{f}=\quad 0.0333$

Restricted Singlet Excited State 15:

Excitation energy $=0.1477744255$ hartrees $\quad 4.02114671 \mathrm{eV} \quad 308.33 \mathrm{~nm}$

$$
\begin{aligned}
& \text { excitation X coeff. } \\
& 195 \Rightarrow 200 \quad 0.11852 \\
& 196 \Rightarrow 201 \quad 0.57620 \\
& 196 \Rightarrow 203 \quad 0.10035 \\
& 196 \Rightarrow 205 \quad 0.11542
\end{aligned}
$$




$$
\begin{aligned}
& 197 \Rightarrow 204 \quad 0.32667 \\
& 197 \Rightarrow 206 \quad-0.66661 \\
& 197 \Rightarrow 209 \quad 0.16729
\end{aligned}
$$

Transition dipole moment (debye):

$$
X=-0.0006 \quad Y=-0.0177 \quad Z=-0.0083 \text { Tot }=0.0196
$$

Oscillator strength, $f=0.0000$ 


\section{Supporting References}

1. AXS, A. 3 B. (2016). Bruker AXS.

2. Krause, L., Herbst-Irmer, R., Sheldrick, G.M., and Stalke, D. (2015). Comparison of silver and molybdenum microfocus X-ray sources for single-crystal structure determination. J. Appl. Crystallogr. 48, 3-10.

3. Sheldrick, G.M. (2015). $\{$ it SHELXT $\}\{--\}$ Integrated space-group and crystal-structure determination. Acta Crystallogr. Sect. A 71, 3-8.

4. Sheldrick, G.M. (2015). Crystal structure refinement with $\{$ it SHELXL\}. Acta Crystallogr. Sect. C 71, 3-8.

5. Wilson, A.J.C., and Geist, V. (1993). International Tables for Crystallography. Volume C: Mathematical, Physical and Chemical Tables. Kluwer Academic Publishers, Dordrecht/Boston/London 1992 (published for the International Union of Crystallography), 883 Seiten, ISBN 0-792-3-16-38X. Cryst. Res. Technol. 28, 110. 